Pure and Applied Mathematics Quarterly

Volume 4, Number 3

(Special Issue: In honor of

Fedor Bogomolov, Part 2 of 2 )

$877-1012,2008$

\title{
Canonical Models of Foliations
}

\author{
Michael McQuillan
}

To Fedya on his 60th birthday

\section{Introduction}

Thirty years after its publication, it remains true that the only absolutely satisfactory theorem on families of curves on surfaces (so a fortiori on higher dimensional) varieties of general type is the conclusion of [B2] that on surfaces with many symmetric tensors curves of a given genus are bounded in moduli. A revealing re-interpretation of this result is provided by Gromov's view of the isoperemetric inequality for (holomorphic) discs as not just a combinatorial consequence of, but as morally equivalent to, negative (holomorphic) sectional curvature. As such, we may re-phrase Bogomolov's theorem as asserting that up to the exception of finitely many rational and elliptic curves the isoperemetric inequality holds for discs in the algebraic directions. Unfortunately, despite the fact that any holomorphic disc may be arbitrarily well approximated by those inside algebraic curves, the isoperemetric inequality that one obtains from Bogomolov's theorem is far too dependent on the genus of the curves in question as to answer even a rather qualitative question such as whether such surfaces admit a Zariski dense

Received January 15, 2007. 
entire mapping. The non-existence of such mappings, i.e. the Green-Griffiths conjecture, would of course be a corollary of the isoperemetric inequality outside of finitely many algebraic curves, and, in-fact, it transpires that they are equivalent, cf. [Kl] and [DV]. In addressing, therefore, the former question for surfaces with many symmetric tensors, the extension [M1] of Bogomolov's theorem to parabolic (in the sense of Ahlfors) Riemann surfaces is very much the child of [B2], while in so much as it was wholly motivated by [M1], the present paper is unquestionably a grandchild.

Underlying any of [B2], [M1] through to our current considerations, is that there is absolutely no sense whatsoever in which algebraic geometry is a priori adapted to the study of sectional curvature. It is, however, extremely good at aiding and abetting the study of Ricci curvature. The critical reason for this is that all the large scale properties of Ricci curvature on a variety $X / \mathbb{C}$ are completely encoded in a single object: the canonical line bundle $K_{X}$ provided the singularities of $X$ are sufficiently nice. In fact, by definition, the largest class of singularities where this assertion holds are known by the name of canonical singularities. That there is a need to restrict one's attention to some class of singularities is evident from the existence of rational plane curves with ample dualising sheaf, that such a class of singularities ought to be exhaustive is the object of the minimal model programme.

The minimal model programme is, of course, a rather old theorem for algebraic surfaces going back at least to Castelnuovo and Enriques. Equally the singularities in question transpire to be extremely simple, i.e. quotients of smooth by finite sub-groups of $\mathrm{SL}_{2}$. From this lemma, one can progress to a more detailed examination of the surface according to the growth of the number of sections of $K_{X}$, i.e. the Kodaira dimension $\kappa(X) \in\{-\infty, 0,1,2\}$. This a priori rather coarse invariant (which takes a sign opposite to the curvature by the way) turns out to be rather precise for $\kappa(X)<2$, and with this hypothesis even allows us to make a list of what might occur. Indeed for $\kappa(X)=-\infty$ we find that the surface must admit a pencil of rational curves, and otherwise there is a canonical model which for $\kappa(X)=0$ looks rather flat, i.e. an abelian, K-3, or Enriques surface, while for $\kappa(X)=1$ it's 'semi-flat', i.e. an elliptic fibration over a hyperbolic (orbi-fold sense) base. Unfortunately the classification theorem's only contribution to the 
remaining case, $\kappa(X)=2$, is an appellation: general type. Nevertheless on the canonical model of such a surface $K_{X}$ is ample so Yau's solution of the Calabi conjecture (in this case strictly speaking Tian-Yau since there may be singularities, $[\mathrm{TY}]$ ) implies that there is a metric with Ricci curvature -1. As such, from the Ricci point of view, our understanding of algebraic surfaces is rather good. In fact, if one adds to this the further insight of Seiberg-Witten theory that $K_{X}$ isn't just a holomorphic but a diffeomorphic invariant, one might even say that our knowledge is excellent.

The problem with the Ricci point of view, however, is that it is really a property of volume not of length. Consequently on canonical models of surfaces of general type one doesn't naturally obtain an isoperemetric inequality for the area of discs in terms of the length of their boundary, but an isoperemetric inequality for the volume of balls in terms of the volume of their boundary. Indeed, this is true even without the solution of the Calabi conjecture, which of itself doesn't improve the situation much beyond the implied constant in the said inequality (and even that isn't improved if one works with a Nevanlinna type volume). Whence for many many years the only real deep geometric insight that came from the Ricci point of view occurred in complex dimension 1, so much so that Gromov could remark, [G1], that the Riemann-Koëbe uniformisation theorem was the Jewel in the Crown of all geometry. Of course this is now radically superseded by Perelman's geometrisation theorem in real dimension 3, albeit that even here we should observe that the (real) dimension is just small enough, and no more, to allow genuinely profound geometric information to be derived from the Ricci tensor.

Now, as it happens, the paper [B2] was (is), in fact, part of a bigger project, cf. [B1], to delve much more deeply into the geometry of algebraic surfaces by way of symmetric tensors. It's very hypothesis that the surface $X$ possesses many symmetric tensors leads to some a priori insight about the (holomorphic) sectional curvature. Indeed the sectional curvature tensor lives rather naturally not on $X$, but on its projective tangent space $\pi: P\left(T_{X}\right)\left(=\mathbb{P}\left(\Omega_{X}\right)\right) \rightarrow X$. The most natural algebraic object on the latter is its tautological line bundle $L$, and $X$ has many symmetric tensors if and only if there is a metricisation $\bar{L}$ of $L$ such 
that for $\omega$ any metric on $X$,

$$
c_{1}(\bar{L}) \gg \pi^{*} \omega
$$

with the inequality in the sense of currents. This may usefully be compared with the definition that the holomorphic sectional curvature of a metric $\omega$ is at most $-K<0$, in which case the particular metricisation $\bar{L}^{\omega}$ induced by $\omega$ satisfies,

$$
\int_{\Delta} f^{*} \omega \leq \frac{1}{K} \int_{\Delta}\left(f^{\prime}\right)^{*} c_{1}\left(\bar{L}^{\omega}\right)
$$

for all holomorphic discs $f: \Delta \rightarrow X$, and of course $f^{\prime}: \Delta \rightarrow P\left(T_{X}\right)$ its derivative. Consequently, Bogomolov's hypothesis is very similar to something like 'generically negative sectional curvature'. In some sense its generically a little stronger, but where the genericity fails it's telling us nothing. Needless to say the failure is occurring at some algebraic hypersurface $S$, say, in $P\left(T_{X}\right)$, and if this hypersurface doesn't dominate $X$ then we certainly have what we're looking for grosso modo: negative sectional curvature outside of finitely many rational and elliptic curves. Consequently the difficulty in the main theorem of [B2] is precisely to prove that curves whose derivatives factor through $S$ admit a bound on their degree, and, effectively, this question poses itself for any surface $X$ and any dominant divisor on the projective tangent space, since there is no longer any help to be had from the global hypothesis on tensors.

Such a question is a lot more tricky than it might seem, since what in effect we'd like to prove is that if enough curves satisfy a given (1st order) differential equation, then they all satisfy an algebraic one. On the other hand, a moment's thought reveals that pulled-back to $S$ the differential equation in question becomes linear of first order, i.e. on $S$ the curves in question are now invariant by a foliation $\mathcal{F}$. At which point, we suddenly find ourselves in the presence of Riccism, because what would be more than sufficient would be to know that sectional curvature along the foliation is negative, and, since its a foliation by (holomorphic) curves this is in fact a Ricci curvature.

To understand the precise functorial properties of the situation, it's extremely helpful to think in terms of an appropriate 2-category of analytic stacks in which the classifying stack $[S / \mathcal{F}]$ is defined. Providing such a definition (e.g. [M5] A.1-2) will not be the object of this introduction nor will it even intervene much in the article beyond a few explanatory notes in V.2 and V.3. Nevertheless, 
it is important to understand that there is a well defined functorial object $\sigma$ : $S \rightarrow[S / \mathcal{F}]$, and a functorially well defined relative dualising sheaf, which for, say $S \mathbb{Q}$-factorial is nothing other than the 1st chern class of the rank 1 quotient of $\Omega_{S}^{1}$ which defines the foliation. Functorially such a bundle should be written $K_{S /[S / \mathcal{F}]}$, but we abbreviate this as $K_{\mathcal{F}}$, the bundle of holomorphic volumes along the leaves. Consequently, and in an absolutely functorial way, we may extend the definitions of Mori theory, and talk about whether $S \rightarrow[S / \mathcal{F}]$ has canonical singularities or not. In the first place we can do this at the level of the algebraic category, while hoping that subsequently such a definition will like the absolute case of varieties mapping to points, truly allow us to encode the properties of the relative (Ricci) curvature in the purely algebraic properties of $K_{\mathcal{F}}$.

Such then will be the programme of this article, but already in the very existence of a modification $\rho: \widetilde{S} \rightarrow S$ such that the induced foliation $\widetilde{\mathcal{F}}$ has canonical singularities we find Bogomolov's solution to the problem of [B2]. Indeed, in any dimension, it isn't that easy to find (formal) invariant curves which pass through the singularities, and in dimension 2 it's particularly tricky, i.e. there are at most 2 such curves, so that if infinitely many algebraic curves were invariant by $\widetilde{\mathcal{F}}$, then all but finitely many of these are pairwise disjoint, so the foliation is necessarily an algebraic pencil.

Much of this line of reasoning continues to apply in the situation of [B2]'s direct descendent [M1], albeit that the curves in question are now maps $f: Y \rightarrow$ $X$ from parabolic Riemann surface. This means that the Green's function viewed from a choice of origin $0 \in Y$ goes to infinity on the ideal boundary, so, in fact, there is a so called Evans potential $p: Y \rightarrow[-\infty, \infty)$ satisfying $d d^{c}(p)=\delta_{0}$, and exhausting $Y$. Instead, therefore, of calculating the degrees of curves, we now seek to calculate the areas of 'discs' $Y(r):=\{y \in Y: p(y)<r\}$, and to bound these areas by their (negative) Euler characteristic, $\chi(r)$, or, equivalently, the zeroes of the meromorphic differential $\partial p$. As it happens, this should all be understood in the sense of Nevanlinna theory, which by its very definitions, cf. [M2], allows much of the argument of [B2] to proceed mutatis mutandis, so for example if $f^{\prime}: Y \rightarrow P\left(T_{X}\right)$ doesn't factor through exactly the same bad $S$ as 
before, one gets an isoperemetric inequality of the form,

$$
\int_{-\infty}^{r} d t \int_{Y(t)} f^{*} \omega \ll-\chi(r)
$$

where, as we've said, the double (Nevanlinna) integral is just a technical device in order to fully profit from the (integrable) complex structure. Consequently, the entire problem of [M1] was to obtain a similar bound under the hypothesis that $f$ was invariant by a foliation $\mathcal{F}$ with (without loss of generality) canonical singularities on a surface $S$. Certainly Bogomolov's theorem permitted us to assume that $f$ was Zariski dense, and with this hypothesis one could prove that canonical singularities, as a priori defined in the algebraic category, exhibited the same excellent properties in greater generality, i.e. up to a very small error,

$$
\int_{-\infty}^{r} d t \int_{Y(t)} f^{*} c_{1}\left(\bar{K}_{\mathcal{F}}\right) \leq-\chi(r)
$$

If one is un-surprised by this, then one is very much overlooking the subtlety of what a canonical singularity is. After all a plane curve $C \rightarrow$ pt is a perfectly good example of a foliation, but if its singularities aren't canonical (equivalently $C$ is smooth since the dimension is 1 ) then this statement is false. Furthermore the a priori definition for foliations is done in an algebraic (and in fact bi-rational) category, so that such a well behaved analytic extension of the definition was by no means obvious. It is, however, true that such analytic extensions are a very very general phenomenon, to such an extent that canonical singularities are exactly those for which such an inequality holds for all invariant discs, and with appropriate (equi-dimensional) changes in definition, this ought not even to depend on the dimension of the leaves.

Such general considerations are not, however, sufficient to prove [M1]. Nevertheless, one does observe that if functorially with respect to the ideas $\sigma: S \rightarrow$ $[S / \mathcal{F}]$ is of general type, i.e. $K_{\mathcal{F}}$ is big, then indeed there is nothing left to do. That this wasn't an entirely ridiculous hypothesis was a theorem of Miyaoka, [Mi2], i.e. if $\mathcal{F}$ isn't a pencil of rational curves than $K_{\mathcal{F}}$ is pseudo-effective. The type of $S$ is, of course, general in [M1], while on a surface we have the index theorem/Zariski decomposition, and excluding $K_{\mathcal{F}}$ contractible (profiting from some of the ideas in [B1]) wasn't hard, so there remained the intermediate possibility that the nef. part $K_{0}$ of $K_{\mathcal{F}}$ was non-trivial yet had zero square. The 
key to excluding this was the intervention of the transverse dynamics, or more precisely that on the étale part of $[S / \mathcal{F}]$ there is a well defined bundle $K_{[S / \mathcal{F}]}$ which extends to a bundle (the chern class of the sub-sheaf defining the foliation) on all of $S$, and satisfying,

$$
K_{S}=K_{\mathcal{F}}+K_{[S / \mathcal{F}]}
$$

Now since $f$ is invariant, this latter bundle ought to intersect $Y$ in zero, but at the singularities of $\mathcal{F}$, canonical or otherwise, the topus isn't étale, and this intersection is calculated by a residue. The conclusion of [M1] was therefore obtained by establishing that this residue vanished as soon as the winding number (functorially understood, this is actually a little different from the multiplicity) of $f$ around the singularities vanished. The winding number being rather easily approximable in an algebraic way, then allowed one to conclude that this was incompatible with the simultaneous possibility of $K_{0}$ having a trivial square and not being able to bound the area of discs in $Y$ by their negative Euler characteristic. Consequently there were, for example, no Zariski dense entire maps invariant by a foliation on a surface of general type, although all the proof really required was $K_{\mathcal{F}} \cdot K_{S}>0$.

It was, however, this very last step involving the transverse dynamic and the calculation of the residue which gave the appearance that [M1] could never proceed beyond the case of surfaces. Furthermore, as the above, only marginally post factum, presentation suggests, the very nature of the problem invited a re-consideration from the point of view of seeking a generalisation of EnriquesKodaira theory, or functorially better Mori theory, to the relative situation of foliations $\sigma: S \rightarrow[S / \mathcal{F}]$. Indeed, modulo it's observations on canonical singularities and curvature, [M1] was trivial for foliations of general type, [Mi] said that (numerical) Kodaira dimension $-\infty$ was equivalent to strictly positive curvature, and even an invariant pencil of rational curves, so why not just classify everything else. Such a classification ought to yield a rather explicit list, and with such a list, there would be no need for the tricky piece of transverse dynamics that had intervened at the end of [M1], and everything would be functorially understood for proceeding to a study of foliations by curves in all dimensions, from which generalisations of [B2], and [M1] to all surfaces, not just those with many symmetric tensors. 
Such was the initial motivation for the study of foliated surfaces by their Kodaira dimension, and it is critical to understand it, because it permeates the introductions of the initial pre-print, [M3], from almost 7 years ago, and what seemed to be a definitive form of it, [M4], 5 years ago. Unfortunately, this supposed definitive version was so caught up in having succeeded in proving a classification theorem (which in some form or another had been a driving pre-occupation since I first read [B2] 14 years ago) that it failed to admit that according to its own motivation it was a complete failure. Indeed rather than re-proving [M1] by a wholly algebraic study in the spirit of Enriques, Kodaira, Mori et al, the entire study hinged on [M1] in an absolutely critical way. That this occurred was the result of the fact that abundance fails for foliations by curves, e.g. quotients of a bi-disc, which isn't a product of curves, come with a pair of foliations, either of which has numerical Kodaira dimension 1, but actual Kodaira dimension $-\infty$. Consequently the classification theorem must wrestle with the study of line bundles $K_{0}$ as encountered in [M1] which are nef., non-torsion, have nil square, but no tensor power of which admits sections. Manifestly this is more or less the exact opposite of what algebraic geometers like to study, and it required radical new methods, with the key to the study being [M1] itself, in fact, a considerable strengthening of it to the case of discs/arbitrary Riemann surfaces rather than just parabolic ones.

One might, therefore, reasonably ask what has changed in the last 5 years, beyond being able to admit that at the last time of writing the introduction was rubbish and failed to admit that a nasty philosophical loop had developed in the motivation. In this respect the principle change is the generalisation of [M1] to foliations by curves in all dimensions, [M7], and in particular an appropriate functorial understanding, [M6], of the measure theory of analytic stacks that eventually permitted a solution of the residue problem in all dimensions. Granted, for the full applicability of the theory to questions such as the GreenGriffiths conjecture one requires to know that every foliation by curves admits a modification with canonical singularities, but this is a detail that isn't at all relevant to the theory itself functorially and categorically understood. Consequently with the certainty of hind sight one can say that the classification theorem is simply an application of a highly functorial piece of methodology, [M1], and this 
is the point of view which we will take. Better still, once one accepts that [M1] is really the key point, then one should apply it freely and liberally, and this leads to a truly satisfactory theory of general type objects, which is properly speaking the generalisation of the uniformisation theorem to this generality, and which goes well beyond the limited detail that algebra alone provides.

With this in mind, a substantive difference between the current article, and the pre-print [M4], is that we will not prove the generalisation of [M1] to arbitrary discs. This has been done elsewhere, and our goal here is to investigate its applications. A similar consideration applies to canonical singularities, at least in so much as more general theorems have already been established, [M5] I.6. A similar remark could be made in respect of foliated minimal models, but, better properties result from studying canonical models, and these I don't know how to construct from 4-folds on. Consequently, there are very good reasons for a hands own approach to the construction of canonical models of foliated surfaces in the usual 'keep track of the contracted graph' sort of way, and this also serves to preserve the historical order in which just recognition is given to the importance of [Mi2] even if it has been improved radically by [BM]. Nevertheless, all but one of the possible graphs lead to quotient singularities on the contraction, so there is a very good case for a systematic use of algebraic stacks (or, if one prefers, since the dimensions are too small for the difference to occasion any substantive problems, orbifolds). Ideally this is what should have been done, but apart from laziness in respect of making too many changes, and warnings from Bogomolov about 'educational issues' associated to algebraic stacks, one should again respect the historical order of the thing, and it wasn't me, but M. Brunella who realised that the contractions being employed in constructing a minimal (as opposed to canonical) model yielded singularities which were the quotients of a smoothly foliated bi-disc by a finite group. This observation subsequently permeated the use of algebraic stacks in the higher dimensional minimal model theorem, [M5], and since its un-questionably the correct point of view (especially in the most delicate aspects of classification) we have made frequent allusions to it, i.e. after presenting the proof more or less as it was, and in a very typically geometry of surfaces sort of way, the technical and conceptual simplification resulting from the use of algebraic stacks is explained. Hopefully the simplifications alone will 
promote a greater interest in stacks which are the natural functorial objects of any geometry from algebraic to the lowest of regularities. Another series of allusions and comments that have been introduced is as to what becomes of the theory on arbitrary foliated 2-dimensional proper stacks, and indeed quasi-projective surfaces. One can conveniently (if not quite technically correctly) view the latter as a stack with infinite monodromy around the boundary, and of itself this makes the quasi-projective case a straightforward interpolation of the proper case which ought to be useful for those engaged in industries such as the study of foliations in the affine plane.

With these remarks in mind, let us make a summary of the chapters. In many points the material is dense, and highly exhaustive in its detail, as was the case of the original pre-prints. These details constitute the most exacting and precise description of the canonical model that the theory allows. This was done, and is being done again with the above stacky extras, so that whatever one wants to know about the behaviour of the curvature of a foliated surface along its leaves should already be here. As such we proceed as follows,

I Singularities. This is an exposition of purely algebraic properties and it strikes me as being difficult to improve the presentation of [M5] I.6 as occasioned by the minimal model theorem in all dimensions. As such, what is of a general nature, i.e. I.1, presents nothing more than a summary of op. cit. On the other hand I.2 is specific to dimension 2, and the main fact here is I.2.4, albeit that knowing these are an exhaustive list of $\mathbb{Q}$-Gorenstein singularities depends on the canonical model theorem. I suspect therefore that some important subtlety about canonical foliation singularities may be being over looked. Indeed like terminal singularities, they must arise from quotients of foliations on smooth surfaces, but the reason for the former is rather obvious I.2.1, while the latter is very nearly false, i.e. fails for log-canonical singularities I.2.5. The final section I.3 discusses residues around singularities, but in a purely algebraic way appropriate to the minimal model theorem. Indeed, much of the way that the notion of invariant bundle and/or curve is employed via residue calculations can be much improved, cf. [M5] II.6, but the actual presentation has the advantage that it intervenes by way of simple numerical calculations akin to those of classical surface theory such as the contractibility of a bunch of curves with negative definite intersection 
matrix, cf. I.3.2.

II Positive Characteristic. The main goal here is the foliated bend and break lemma of Miyaoka, II.4.1, which on surfaces easily yields the cone theorem II.4.3. The alternative approach of Bogomolov and myself that works in all dimensions is summarised in II.5. The pre-ceding sections, especially II.1 and II.2 have a much more ambitious goal by way of the role of positive characteristic methods in classification. As such where they might lead can only really be understood after the final section V.5.

III Minimal models. The object here isn't just a minimal model theorem, i.e. a model with $K_{\mathcal{F}}$ nef for $\mathcal{F}$ not a rational pencil, III.2.1, but to make $K_{\mathcal{F}}$ as positive as possible, i.e. a canonical model as defined III.3.1, whose existence and structure is detailed in III.3.2.

IV Classification. This is the central part of the manuscript, and merits division by the numerical foliated Kodaira dimension $\nu(\mathcal{F})$, viz:

IV.1 $\nu(\mathcal{F})=-\infty$. By Miyaoka's theorem this implies that the foliation is a conic pencil, but the converse is only true with canonical singularities. The log-canonical situation is, however, rather interesting, and is a nice example of the naturality of algebraic stacks IV.1.4.

IV.2 $\nu(\mathcal{F})=2$. The important thing here is the lack of a good algebraic description, i.e. the canonical ring need not be finitely generated IV.2.3. This results from the structure, IV.2.2, of the non $\mathbb{Q}$-Gorenstein (so understood in the sense of Mumford intersection theory) singularities. For the ambient space a surface, this failure is specific to non-integrable foliations, but the spirit of the counterexample is similar to its failure in the integrable case in higher dimensions, cf. $[\mathrm{Ke}] 3.1$.

IV.3 $\nu(\mathcal{F})=0$. As might be expected these arise from vector fields, but may actually be a quotient under a finite group action preserving the foliation, but not the field. A complete list of possibilities is provided in IV.3.6.

IV. $4 \nu(\mathcal{F})=1$, and $\kappa(\mathcal{F}) \geq 0$. This goes very much as expected, i.e. abundance holds, and there is a Kodaira fibration, IV.5.1. If anything the proof is easier than its classical Enriques analogue since the presence of the foliation is a 
help not a hindrance. In any case, apart from elliptic pencils of varying moduli, the fibration is transverse in all but finitely many fibres to the foliation. Whence, outside of the said fibres, such foliations permit a very elegant description of $[X / \mathcal{F}]$ as a conic, elliptic or higher genus bundle over an appropriate $B \Gamma$, for $\Gamma$ discrete. Nevertheless, in the conic or elliptic cases, so called Riccatti or Turbulent foliations, there's much more to the canonical model, and indeed a further compactification data at the non-transverse fibres is required. This is explained in detail in IV.4.4, with the complications of the Riccatti case, IV.4.4.(ii) being particularly noteworthy since they are shadows of yet more complicated phenomenon in the situation of general type.

IV.5 $\nu(\mathcal{F})=1$, and $\kappa(\mathcal{F})=-\infty$. Ultimately, IV.5.11, the canonical models will transpire to be the Baily-Borel compactification of Hilbert-Modular surfaces in either of their natural foliations. The proof appears arduous, so one should read the more general discussion (extending to general type foliations) of variation of conformal structure in V.3 first to understand that the principle is rather simple. Alternatively, the history of the proof reveals that its complication is more imagined than real. Specifically, the very first version [M3] of this article addressed the question under the hypothesis that $K_{\mathcal{F}}$ admitted a sufficiently regular semi-positive metricisation $\bar{K}_{\mathcal{F}}$. Under such a hypothesis one might hope to identify another foliation $\mathcal{G}$ transverse to $\mathcal{F}$ as the kernel of the semi-positive form $c_{1}\left(\bar{K}_{\mathcal{F}}\right)$. Any expert on such so called Monge-Ampère foliations will tell you, however, that such a hope is vain, and this is a waste of time. Surprisingly, but rather easily, the entire theory of such a Monge-Ampère foliation $\mathcal{G}$ radically simplifies in the presence of $\mathcal{F}$, and this is just yet another example of the power of the Bochner technique/integration by parts, and is a very general observation in all dimensions. In any case, the problem seemed to be solved, but, unfortunately the desired regularity of the metric was rather high, e.g. local weights with at least 2nd derivatives $\ell_{2}$, and general nonsense was never going to produce anything beyond first derivatives $\ell_{2-\epsilon}$. The solution to this difficulty was proposed by M. Brunella, i.e. one should take the canonical Poincaré metric guaranteed by [M1] and the uniformisation theorem whose curvature along the leaves is -1 . A priori, however, this might not have been semi-positive, and establishing this is Brunella's theorem [Br5]. The notable feature of this choice is that its symmetries 
force a lot of cancellation in the Bochner formula, which in fact becomes local, and in turn the required regularity is much less, in fact, one sees that locally bounded will do. As such, the theorem was completed when [M1] was generalised from parabolic to arbitrary Riemann surfaces. Whence, since the scope of this article is applications of [M1], the contents of IV.5 (modulo the initial overlap with IV.4) are the wholly generalisable Bochner formula, and the proof, by way of a regularisation argument, that the said Bochner formalism works for the leaf wise Poincaré metric in the presence of Brunella's semi-positivity theorem (which is logically indispensable so it's worth noting Brunella's second proof [Br6]), and some appropriate generalisation of [M1].

V Conformal structure. More or less by definition a choice of metricisation on $T_{\mathcal{F}}$ allows us to consider $\sigma: S \rightarrow[S / \mathcal{F}]$ as a varying family of conformal structures. Plainly the best choice is the canonical or Poincaré metric. This may, however, not always make sense, e.g. when the foliation is given by a global vector field. In fact, this and rational or elliptic pencils is essentially the only way that the canonical metric isn't well defined and continuous outside a proper algebraic set, V.1. Nevertheless there is a subtlety in the use of 'essentially', V.1.4, since some of the foliations in question are not quite those of Kodaira dimension 0 , but are actually bi-analytic, as opposed to bi-rational compactifications of them, V.2, so that the Kodaira dimension may actually be 1 , albeit that most things of Kodaira dimension 1 are hyperbolic. As such, there is a further refinement of classification via Kodaira dimension by way of (uniform) hyperbolicity of the leaves. In such a refinement, what is seen to distinguish the hyperbolic foliations of Kodaira dimension 1 from general type is that the conformal structure of the latter varies, where this should be understood, V.3, in a natural generalisation of the Weil-Petersson metric. In addition, we see that the combination of Brunella's semi-positivity theorem and [M1] allows a not un-reasonable generalisation of Bers simultaneous uniformisation theorem to the situation of $\sigma: S \rightarrow[S / \mathcal{F}]$, V.3. To conclude, therefore, given its central role, it seems appropriate to review not only the proof of [M1] (and in its generalised form) but exactly how it naturally occurs as a substitute for abundance given the failure of the latter, V.4. Finally, V.5, we review what can be retained in positive and mixed characteristic. This review reveals that in so much as hyperbolicity 
problems are concerned (in characteristic zero) even the proof of [M1] enjoys a certain optimality, V.5.6, although this doesn't quite exclude the possibility (as dictated by the original motivation and on which Bogomolov and I have devoted some effort) of a proof of the classification theorem (but not of [M1]) by way of positive characteristic methods, V.5.8.

Certainly, Bogomolov himself has taken a considerable interest in this article, and its development, so it's not only a pleasure to thank him for his contributions, but also to offer the article to him on the occasion of the celebration of his 60th birthday. Another important contributor, beyond even his theorem on the pluri-sub-harmonic variation of the Poincaré metric, was Brunella, to whom along with Gromov, Mendes, and Shepherd-Barron grateful thanks are extended. More critical, however, was the contribution of Cécile in preparing the text, and my apologies to her for any lack of elegance that my clumsy editing may have introduced. 


\section{Singularities}

\section{I.1. A summary}

Somewhat surprisingly the theory of minimal models of foliations on surfaces necessitates a consideration of singularities very much in the spirit of Mori's programme for 3 -folds. Although we will ultimately be occupied only with what may occur in dimension 2 there seems to be no harm in setting out from a more general perspective. Our basic objects of study are pairs $(X, \mathcal{F})$ where $X$ is a normal projective variety (or possibly just a proper normal algebraic space) over a field $k$, and $\mathcal{F}$ is an integrable foliation, i.e. a saturated subsheaf $\mathcal{T}_{\mathcal{F}}$ of the tangent sheaf $\mathcal{T}_{X}$ closed under lie brackets. Necessarily there is an immersion $j:\left(X_{0}, \mathcal{F}_{0}\right) \hookrightarrow(X, \mathcal{F})$ with $X \backslash X_{0}$ of codimension $2, X_{0}$ non-singular and $\mathcal{T}_{\mathcal{F}_{0}}$ locally free so that we may define $K_{\mathcal{F}}$ to be $j_{*}\left(\Lambda^{\text {top }} \mathcal{T}_{\mathcal{F}_{0}}^{\vee}\right)$. Of course if we wish to pursue the study of such objects in the spirit of the minimal model programme then $K_{\mathcal{F}}$ better be $\mathbb{Q}$-Cartier. The strongest possible sense in which this may be understood is naturally,

Definition I.1.1. A pair $(X, \mathcal{F})$ is said to be $\mathbb{Q}$-foliated Gorenstein if there exists $m \in \mathbb{N}$ such that $j_{*}\left\{\left(\Lambda^{\text {top }} \mathcal{T}_{\mathcal{F}}^{\vee}\right)^{\otimes m}\right\}$ is locally free. If in addition we can take $m=1$, then we say that $(X, \mathcal{F})$ is foliated Gorenstein.

Now the important thing to remark is that there is a priori no relation between the singularities of $X$ and those of $(X, \mathcal{F})$. Indeed the curious reader may skip to IV.2.2 to see an example where the underlying space is Gorenstein but $(X, \mathcal{F})$ is not foliated Gorenstein. Conversely we suppose nothing about $X$ beyond its normality. Regardless, as we will see shortly there are other weaker interpretations of the notion of $K_{\mathcal{F}}$ being $\mathbb{Q}$-Cartier so let us simply be a little vague about it for the moment and continue in the usual way, [K2], i.e., let $p:(\widetilde{X}, \widetilde{\mathcal{F}}) \rightarrow(X, \mathcal{F})$ be a proper birational morphism of pairs, with the obvious definition of that notion, then in $\operatorname{NS}^{1}(\widetilde{X})_{\mathbb{Q}}$ we may write,

$$
K_{\widetilde{\mathcal{F}}}=p^{*} K_{\mathcal{F}}+\sum_{E} a(E, X, \mathcal{F}) E
$$

for $E$ an exceptional divisor contracted by $p$. Should $p^{\prime}:\left(X^{\prime}, \mathcal{F}^{\prime}\right) \rightarrow(X, \mathcal{F})$ be another such map then for any "reasonable" definition of $K_{\mathcal{F}}$ being $\mathbb{Q}$-Cartier 
(e.g. $(X, \mathcal{F}) \mathbb{Q}$-foliated Gorenstein) then given $E$ in $\widetilde{X}$ with non-empty proper transform $E^{\prime}$ we have $a(E, X, \mathcal{F})=a\left(E^{\prime}, X^{\prime}, \mathcal{F}^{\prime}\right)$, whence we obtain a map,

$$
a(-, X, \mathcal{F}):\{\text { exceptional divisors over } X\} \rightarrow \mathbb{Q}
$$

There is obviously no harm in extending $a_{X, \mathcal{F}}$ to actual divisors on $X$ by putting it to be identically zero on the same. Indeed this is even internally consistent since $\mathcal{T}_{\mathcal{F}}$ is saturated. At this point everything translates rather nicely into commutative algebra. Any divisor on any modification of $X$ certainly defines a discrete rank 1 valuation on the field of rational functions, and by [ZS], Theorem 31, the converse is true if we restrict to valuations with non-empty centre (n.b. this condition is vacuous if $X$ is proper) whose residue field over the residue field of the centre has transcendence degree at least the dimension of the local ring of the centre minus 1 , i.e. so called prime divisors,

Our function $a_{X, \mathcal{F}}$ is therefore a function,

$$
a_{X, \mathcal{F}}:\{\text { prime divisors of } k(X) \text { with non-empty centre }\} \rightarrow \mathbb{Q}
$$

and we may thus define,

Definition I.1.2. Let $Z$ be an irreducible subscheme of $X$, then,

$$
\operatorname{discrep}_{X, \mathcal{F}}(Z)=\inf \left\{a_{X, \mathcal{F}}(v): \text { centre of } v \supset Z\right\}
$$

Furthermore we say (by analogy with the usual case) that $(X, \mathcal{F})$ has a canonical singularity at $Z$ (respectively terminal) if the discrepancy, $\operatorname{discrep}_{X, \mathcal{F}}(Z)$, is nonnegative (respectively strictly positive).

It's worth noting,

Remark I.1.3. While this definition could have been phrased more precisely as say canonical foliated singularities (resp. terminal foliated singularities) we have chosen not to do so since the usual notions of canonical and terminal make no appearance in this article. Whence the words canonical, and terminal should always be understood as canonical foliated and terminal foliated.

Now in commutative algebra terms here's what's going on. At the level of the local ring $\mathcal{O}_{X, Z}$ we have a saturated submodule $\mathcal{T}_{\mathcal{F}} \otimes \mathcal{O}_{X, Z}$ of $\operatorname{Der}_{k}\left(\mathcal{O}_{X, Z}\right)$ 
$\left(=\mathcal{O}_{X, Z} \otimes \mathcal{T}_{X}\right.$, by the way, since dualising coherent modules commutes with flat base change). A discrete rank 1 valuation $v$ with centre containing $Z$ corresponds to a not necessarily dominant map of local rings $\mathcal{O}_{X, Z} \rightarrow R$, where $R \subset k(X)$ is a discrete valuation ring with valuation $v$. There is then a unique maximal, and indeed free, submodule $T_{\widetilde{\mathcal{F}}}$ of $\operatorname{Der}_{k}(R)$ with $T_{\widetilde{\mathcal{F}}} \otimes_{R} k(X)=\mathcal{T}_{\mathcal{F}} \otimes_{\mathcal{O}_{X}} k(X)$ (strictly speaking we should sheafify the left hand side, but it's notationally convenient to identify modules and their sheafication, so we will). Interpreting negative powers of the maximal ideal of $R$ in the obvious way there is an integer $d$ with,

$$
R \otimes \mathcal{O}_{X, Z} \operatorname{Hom}\left(\left(\Lambda^{\text {top }} \mathcal{T}_{\mathcal{F}} \otimes \mathcal{O}_{X, Z}\right)^{\otimes m}, \mathcal{O}_{X, Z}\right)=\mathfrak{m}_{R}^{d} \otimes\left(\Lambda^{\text {top }} T_{\widetilde{\mathcal{F}}}^{\vee}\right)^{\otimes m}
$$

where $m$ is the appropriate power for making $K_{\mathcal{F}}$ a bundle and the integer $d$ is of course the discrepancy. Unfortunately this definition is not terribly practical if the foliation isn't Gorenstein, i.e. the relation between duals and tensor products is a mess. However in the Gorenstein case it's all rather tidy. Let's illustrate this for foliations by curves. Since we've assumed that $X$ is normal, this is equivalent to $\mathcal{T}_{\mathcal{F}}$ being a bundle, i.e. the foliation is given everywhere by a possibly singular vector field. Naturally we take a vector field $\partial$ which generates $\mathcal{T}_{\mathcal{F}}$ generically around $Z$. Equally there is a derivation $\widetilde{\partial}$ of $R$ generating $T_{\widetilde{\mathcal{F}}}$ so that if $\pi$ is a uniformising parameter then, $\widetilde{\partial}=\pi^{d} \partial$. These observations allow us to start proving some properties of canonical singularities, albeit we begin with what we mean by a smooth point,

Definition I.1.4. A (scheme) point $Z$ of a foliation by curves $(X, \mathcal{F})$ is said to be smooth if locally around $Z, \mathcal{T}_{\mathcal{F}}=\mathcal{O}_{X, Z} \partial$ for $\partial$ a derivation whose residue in $T_{M} \otimes k(Z)$ is non-zero for some ambient non-singular variety $M$ in which $X$ is embedded around $Z$. The singular points, $\operatorname{sing}(\mathcal{F})$, is the set of non-smooth points.

Of course it might be linguistically preferable to call a singular point a nonsmooth point, since the notion of canonical singularity doesn't suppose that the point is singular in the above sense. On the other hand, this kind of convention is well established in the Mori theory framework, so no confusion seems likely. It's also worth remarking that a smooth point is Gorenstein, and this latter condition is open for general nonsense reasons, so openness of the smooth locus follows from its openness in the Gorenstein locus, which is clear. In characteristic zero smoothness at $Z$ is equivalent, by the classical Frobenius theorem, to the rather more 
descriptive property of the foliation being given in a formal (or even analytic) neighbourhood of $Z$ by an everywhere relatively smooth fibration. Better still, the Frobenius theorem may even be interpreted as defining a smooth infinitesimal equivalence relation at the smooth points of the foliation, which in turn, admits an analytic continuation to a smooth groupoid in the analytic topology, and even (after some blowing up) an extension of the same over the singular points (albeit, very probably, with non-discrete stabilisers at such points), so that in an appropriate 2-category of analytic stacks, a foliation in characteristic zero is best thought of in terms of the quotient map $X \rightarrow[X / \mathcal{F}]$ of $X$ over the classifying stack of the foliation. From this latter point of view, the definitions that we have proposed are nothing other than the relativisation of the standard definitions of standard Mori theory.

Needless to say we have so far refrained from a discussion of the more general notions of log-terminal and log-canonical. To this end let us simply localise our discussion by putting $X=\operatorname{Spec} \mathcal{O}_{X, Z}$, and introduce finitely many effective $\mathbb{Q}$ Cartier prime divisors $B_{i}$. To such divisors, or more generally prime divisors, $E$, of the function field, we must introduce a function,

$$
\epsilon(E):=\left\{\begin{array}{rr}
0 & \text { if } E \text { is invariant by the foliation } \\
1 & \text { if } E \text { is not invairant by the foliation }
\end{array}\right.
$$

where foliation should be understood as the saturation of our given foliation about the local ring at $E$. For convenience we'll put $\epsilon_{i}=\epsilon\left(B_{i}\right)$, and introduce weights $0<a_{i} \leq 1$ associated to each $B_{i}$, which, in turn defines the boundary,

$$
B:=\sum_{i} a_{i} \epsilon_{i} B_{i}
$$

Furthermore, at any prime divisor, $E, B_{i}$ affords a multiplicity $\nu_{i}(E)$, and we make,

Definition I.1.5. The germ $(X, B, F)$ is said to enjoy any of the properties listed below if for every prime divisor $E$ with centre $Z$ the appropriate inequality holds,

(a) Terminal whenever $a_{X, \mathcal{F}}(E)-\sum a_{i} \epsilon_{i} \nu_{i}(E)>0$.

(b) Canonical whenever $a_{X, \mathcal{F}}(E)-\sum a_{i} \epsilon_{i} \nu_{i}(E) \geq 0$. 
(c) Log-terminal whenever $a_{X, \mathcal{F}}(E)-\sum a_{i} \epsilon_{i} \nu_{i}(E)>-\epsilon(E)$.

(d) Log-canonical whenever $a_{X, \mathcal{F}}(E)-\sum a_{i} \epsilon_{i} \nu_{i}(E) \geq-\epsilon(E)$.

Once again, these are the functorial extensions of standard Mori theory to the relative situation $X \rightarrow[X / \mathcal{F}]$. The inevitable intervention of the function $\epsilon$ can be seem by extracting roots of divisors, or looking at how $T_{\mathcal{F}}$ transforms as a subsheaf of the sheaf of derivations with logarithmic zeroes, provided the $a_{i}$ have the form $1-\frac{1}{e_{i}}$ for $e_{i} \in \mathbb{N} \cup\{\infty\}$. In any case we can, evidently, without loss of generality suppose that all of the $B_{i}$ are transverse to the foliation at their generic point.

All of which is as true for an arbitrary foliation, as it is for a foliation by curves. However, just as a curve singularity which is any of terminal, canonical, etc. is rather well behaved, so too do these definitions behave well for foliations by curves. In the first instance this is most easily done when everything is Gorenstein, so, also the $B_{i}$ Cartier, and we summarise the salient facts from [M5] I.6.

Fact I.1.6. Let $(X, \mathcal{F})$ be the germ of a Gorenstein foliation by curves in characteristic zero then it is terminal if and only if it is smooth with $Z$ transverse to $\mathcal{F}$.

Proof. op. cit. I.6.9 $\square$

Which leads to,

Fact I.1.7. Let $(X, B, \mathcal{F})$ be the germ of a Gorenstein foliation by curves with Cartier boundary, then in characteristic zero the following are equivalent,

(a) $(X, B, \mathcal{F})$ is terminal.

(b) $(X, B, \mathcal{F})$ is log-terminal.

(c) Either $B$ consists of a single component of multiplicity 1 transverse to $\mathcal{F}$ and of weight $<1$, or $B$ is empty, and in both cases $(X, \mathcal{F})$ is terminal.

Proof. op. cit. I.6.11

To describe the canonical and log-canonical singularities requires a little notation. As in I.1.4, we fix an embedding $X \hookrightarrow M$ of $X$ into a smooth $M$ (of 
which everything will be ultimately independent), and, furthermore choose a quasi-coefficient field $K$ in $\mathcal{O}_{M, Z}$ of $k(Z)$. This allows us to decompose a vector field $\partial$ defining the foliation as,

$$
\partial=\partial_{K}+\delta
$$

where the latter is a $k$ derivation of $K$, and the former a $K$ derivation of $\mathcal{O}_{M, Z}$. All of which may look highly non-functorial, but on working to first order at singular (more precisely non-smooth) points, functoriality is restored, and we obtain a $K$ linear map,

$$
D_{K} \in \operatorname{End}\left(\frac{\mathfrak{m}_{X, Z}}{\mathfrak{m}_{X, Z}^{2}}\right)
$$

As such, we can talk about $D_{K}$ being semi-simple, nilpotent etc. as a $K$ linear map, and indeed,

Fact I.1.8. Let $(X, \mathcal{F})$ be the germ of a singular Gorenstein foliation by curves then it is log-canonical iff $D_{K}$ is non-nilpotent.

Proof. op. cit. I.6.13

Unlike I.1.7, log-canonical and canonical are not quite identical, even for an empty boundary, but the difference (at least algebraically speaking) is slight,

Fact I.1.9. A singular Gorenstein germ $(X, \mathcal{F})$ which is log-canonical is canonical unless it satisfies one of the following equivalent conditions,

(a) There are $K$ embedding coordinates, $x_{1}, \cdots, x_{d}$, and $m_{1}, \cdots, m_{d}$, positive integers, such that up to re-scaling by a unit,

$$
\partial_{K}=m_{1} x_{1} \frac{\partial}{\partial x_{1}}+\cdots+m_{d} x_{d} \frac{\partial}{\partial x_{d}}
$$

(b) $Z$ is the centre of a prime valuation with negative $(X, \mathcal{F})$ discrepancy.

(c) $Z$ is the centre of a prime valuation $E$ with $(X, \mathcal{F})$ discrepancy -1 and $\epsilon(E)=1$.

Proof. op. cit. I.6.12

As it happens, the discussion becomes a little more complicated if the weights $a_{i}<\frac{1}{2}$, and to the best of my knowledge there is no particular reason for working 
in this generality. Indeed the natural cases are weights of the form $1-\frac{1}{e_{i}}$, for $e_{i}$ a positive integer, which correspond to coverings, or $e_{i}=\infty$ which corresponds to quasi- projective varieties and/or coverings by logarithms. Let us call such weights geometric and observe,

Fact I.1.10. Let $(X, B, \mathcal{F})$ be the germ of a Gorenstein foliation by curves in characteristic zero with non-empty Cartier boundary, and geometric weights which is log-canonical then, in fact, it is canonical, and should it not be terminal the possibilities are,

(a) The germ $(X, \mathcal{F})$ is terminal, $B$ has multiplicity 1 and weight 1.

(b) The germ $(X, \mathcal{F})$ is terminal, $B$ has multiplicity 1 and weight $\frac{1}{2}$, and enjoys a simple tangency with $\mathcal{F}$, i.e. if $\partial$ and $f$ are local equations $\partial^{2}(f) \neq 0$.

Proof op. cit. I.6.14

All of which is a rather exhaustive description of the Gorenstein case. To go from Gorenstein to $\mathbb{Q}$-Gorenstein one employs in characteristic zero the standard covering trick by way of extracting roots, i.e.,

Triviality I.1.11. Let $(X, \mathcal{F})$ be a normal $\mathbb{Q}$-Gorenstein germ over $\mathbb{C}$, then there is a cyclic covering $p: Y \rightarrow X$, étale in codimension 1 such that the induced foliation $(Y, \mathcal{F})$ is Gorenstein. Better still this construction globalises, so that for $(X, \mathcal{F})$ normal $\mathbb{Q}$-Gorenstein there is a smallest normal Deligne-Mumford stack $\pi: \mathcal{X} \rightarrow X$, the Gorenstein covering stack, étale in co-dimension 1, such that the induced foliation $(\mathcal{X}, \mathcal{F})$ is Gorenstein.

Again the details are in op. cit., this time I.5. Unfortunately, the stack word often leads to a reaction of blind panic. Post $[\mathrm{KM}]$, however, this is completely un-warranted (cf. [M5] I.2-3), since locally (in the étale topology) a stack is just a finite group acting on a scheme. In particular, in the context of I.1.11, this means that one shouldn't just think of $Y$, or for that matter $(Y, \mathcal{F})$, but of $Y$ together with the action of $\operatorname{gal}(Y / X)$, whose fixed points are the locus where $p$ isn't étale, and, of course, $Y$ becomes an étale neighbourhood of $\mathcal{X}$. Consequently, even locally it is more functorial to think of $\mathcal{X}$ than $Y$, and in contra-distinction with ordinary Mori theory we have, 
Fact I.1.12. Let $(X, \mathcal{F})$ be a germ of a $\mathbb{Q}$-Gorenstein foliation by curves in characteristic zero with $\pi:(\mathcal{X}, \mathcal{F}) \rightarrow(X, \mathcal{F})$ its Gorenstein covering stack, then $(X, \mathcal{F})$ has terminal, respectively canonical, respectively etc. singularities iff $(\mathcal{X}, \mathcal{F})$ does.

Proof op. cit. I.7.1 $\square$

Needless to say this discussion equally extends to the case of a boundary divisor, albeit that one should be careful to extract the roots of the $B_{i}$ one at a time, so as to get a stack $\widetilde{\mathcal{X}} \rightarrow \mathcal{X}$, étale in co-dimension 1 , which not only has the induced foliation Gorenstein but on which each $B_{i}$ becomes Cartier. Again the relation between terminal, canonical etc. on $(\widetilde{\mathcal{X}}, B, \mathcal{F})$ and the same on $(X, B, \mathcal{F})$ is, as per I.1.12, necessary and sufficient, so that I.1.6-10 also give an exhaustive description in the $\mathbb{Q}$-Gorenstein case by simply observing that the latter is the quotient of the former by a finite group. At which point we may reasonably close this section by way of,

Remark I.1.13. (cf. op. cit. I.7) It isn't really our intention to trouble ourselves overtly with non-empty boundary divisors. Nevertheless, there is quite a lot of practical interest in the case where the boundary weights are all 1 . The easiest way to go from a non-empty boundary, to what in some sense might be termed a full boundary is to use covering stacks. Indeed, given a boundary with geometric weights $<1$ we can construct a stack $\xi: \Xi \rightarrow X$, ramified only over the $B_{i}$ in co-dimension 1 such that the induced foliation $\mathcal{G}$ on $\Xi$ has canonical bundle,

$$
K_{\mathcal{G}}=\xi^{*} K_{\mathcal{F}}+\sum_{i} a_{i} \xi^{*} B_{i}
$$

so that minimal model theory on $\Xi$ without boundary is simply that of $X$ with boundary. Unfortunately, this doesn't quite work if the $a_{i}=1$, but, needless to say, the $a_{i}=1$ case is often easily deducible from the $a_{i}<1$ case by the simple expedient of considering the limiting behaviour. As such, it's quite useful to think of the $a_{i}=1$ case as a stack with countable stabilisers along the $B_{i}$ even though such a thing doesn't quite exist.

\section{I.2. Dimension 2}

As one might imagine, dimension 2 presents a number of both simplifying and particular features. Our discussion will continue to be local, so that $X=$ 
$\operatorname{Spec} \mathcal{O}_{X, Z}$ is a local normal germ with a foliation by curves $\mathcal{F}$, although we'll now impose that $k(X)$ has transcendence degree 2 over, for simplicity, an algebraically closed base field $k$, with $Z$ now a closed point. In particular we can immediately note,

Fact I.2.1. (characteristic 0) Let $(X, \mathcal{F})$ be a foliated Gorenstein normal surface germ which is terminal, then $X$ is smooth.

Proof It suffices to prove that the completion $\hat{X}$ in $Z$ is smooth. On the other by I.1.6 hand and the Frobenius theorem, there is a fine quotient $\hat{X} \rightarrow C:=[\hat{X} / \mathcal{F}]$, where $C$ is a normal 1 dimensional formal scheme. Consequently $C$ is smooth, while $\hat{X} \rightarrow C$ is a smooth fibration.

From which we can explicitly describe all terminal surface singularities by way of,

Corollary I.2.2. (characteristic 0$)$ Let $(X, \mathcal{F})$ be a foliated $\mathbb{Q}$-Gorenstein normal surface germ which is terminal, then for some $n \in \mathbb{N}$ it is the quotient of a smoothly foliated smooth germ $(Y, \mathcal{F})$ by an action of $\mathbb{Z} / n$ preserving both the fixed point and the foliation. In particular, on $Y$ there are coordinates $x, y$ and faithful characters $\chi, \psi$ of $\mathbb{Z} / n$, such that the foliation is given by,

$$
\partial=\frac{\partial}{\partial x}
$$

and for $\sigma \in \mathbb{Z} / n$ the action by,

$$
\sigma: Y \longrightarrow Y: x \mapsto \chi(\sigma) x, y \mapsto \psi(\sigma) y
$$

Proof The first part is just a restatement of I.1.12 and I.2.1. Necessarily, however, the action of $\mathbb{Z} / n$ descends to the $C$ of the proof of I.2.1, so the existence of the coordinate $y$ with actions of the foliation and of the group as described is clear. The existence of $\partial$ follows from the usual identification of the local Picard group with characters, or indeed the very construction of $Y$, which allows us to find a generator $\partial$ of the foliation such that, $\partial^{\sigma}=\chi(\sigma) \partial$ for some faithful character $\chi$. Appealing to the Frobenius theorem, we can find $\xi$ such that $\partial \xi=1$, so $\sigma$ sends $\xi$ to $\chi(\sigma) \xi+f(y)$, for some function $f$ of $y$. Next one introduces a variable, $x=\xi+g(y)$, for a suitable $g$, so that the action on $x$ is linear. Indeed finding such a $x$ involves solving linear equations, all of which can be solved since the 
group action is finite cyclic. Finally, by construction the group action is without pseudo reflections, so both the characters must be faithful.

One can even observe that the coordinates of I.2.2 converge in the analytic topology, so the conclusion of this is that terminal foliation singularities on surfaces are as clean as it gets. Now as it happens, the ambient space of a canonical $\mathbb{Q}$-Gorenstein foliated singularity on a surface is never worse than a quotient singularity, and rather particular ones at that. Nevertheless, I don't know any direct proof of this in the spirit of I.2.1, as opposed to brute force calculation by way of resolutions. Let us, however, anticipate this development (III.3.2), and proceed to a description of the local possibilities. To this end let us recall,

Reminder I.2.3. (cf. [Ma]) let $A$ be a complete regular local ring containing a coefficient field, $L$, supposed algebraically closed. Next let $\partial \in \mathfrak{m} \operatorname{Der}_{L}(A)$. For every $n \in \mathbb{N}$ we have an exact sequence,

$$
0 \longrightarrow \frac{\mathfrak{m}^{n}}{\mathfrak{m}^{n+1}} \longrightarrow \frac{A}{\mathfrak{m}^{n+1}} \longrightarrow \frac{A}{\mathfrak{m}^{n}} \longrightarrow 0 .
$$

We can consider $\partial$ as a $L$-linear endomorphism, $\partial_{n}$, of $A_{n}=A / \mathfrak{m}^{n}$ for each $n$. Consequently $\partial_{n}$ has a Jordan decomposition $\partial_{S, n} \oplus \partial_{N, n}$ into a semi-simple and nilpotent part. These are compatible with the restriction maps $A_{n+1} \rightarrow A_{n}$, and so on taking limits give a Jordan decomposition $\partial_{S} \oplus \partial_{N}$ of $\partial$. In particular $\partial$ is semi-simple iff there is a choice of generators $x_{i} \in \mathfrak{m}$, together with $\lambda_{i} \in L$ such that,

$$
\partial=\sum_{i} \lambda_{i} x_{i} \frac{\partial}{\partial x_{i}}
$$

To describe the nilpotent part, observe that $\left[\partial_{S}, \partial_{N}\right]=0$, so given $\partial_{S}$ as above we just compute a basis for fields which commute with it. Putting $\Lambda=\left(\lambda_{1}, \ldots, \lambda_{n}\right)$ and $\Lambda \cdot-$, to be the usual inner product these are easily seen to be, cf. op.cit. ${ }^{1}$,

(a) $x^{Q} x_{i} \frac{\partial}{\partial x_{i}}, \Lambda \cdot Q=0, Q=\left(q_{1}, \ldots, q_{n}\right), q_{j} \in \mathbb{N} \cup\{0\}$

(b) $x^{Q} x_{i} \frac{\partial}{\partial x_{i}}, \Lambda \cdot Q=0, Q=\left(q_{1}, \ldots, q_{n}\right), q_{j} \in \mathbb{N} \cup\{0\}$ for $j \neq i, q_{i}=-1$

\footnotetext{
${ }^{1}$ The situation in op.cit. is only discussed in characteristic zero. However what is at stake is just some linear algebra which is valid in any characteristic provided $\Lambda \cdot Q$ is understood to take values in $L$.
} 
where of course $x^{Q}=x_{1}^{q_{1}} \ldots x_{n}^{q_{n}}$.

In the immediate case to hand, $A$ will be $\mathbb{C}[[x, y]]$, and there is, up to scaling, only one eigenvalue, $\lambda$, say, so an exhaustive, and rather small, list of formal normal forms in dimension 2 is easily constructed. If, however, our original singularity of interest was canonical and $\mathbb{Q}$-Gorenstein, we must also allow for an action of a finite group $G$, acting on $\partial$ by a possibly trivial, and not necessarily faithful, character $\theta$, i.e. $\partial^{\sigma}=\theta(\sigma) \partial$, for $\sigma \in G$. The action of $G$ must, however, preserve the Jordan decomposition, so, in fact, $\theta$ is either trivial or takes values in $\mathbb{Z} / 2$. Combining these observations with I.2.3 and anticipating III.3.2 allows us to assert,

Fact I.2.4. A $\mathbb{Q}$-foliated Gorenstein singularity in characteristic zero which is not terminal, but nevertheless canonical is formally one of the following,

(a) The quotient by $\mathbb{Z} / n$ of $\partial=x \frac{\partial}{\partial x}+\lambda y \frac{\partial}{\partial y}$, where $\lambda \notin \mathbb{Q}$, and the group acts by, $\sigma: x \mapsto \chi(\sigma) x, y \mapsto \psi(\sigma) y$, for some faithful characters $\chi$ and $\psi$.

(b) The quotient by $\mathbb{Z} / n$ of $\partial=x \frac{\partial}{\partial x}+\left(\lambda y+x^{\lambda}\right) \frac{\partial}{\partial y}$, where $\lambda \in \mathbb{N}$, and the group acts by, $\sigma: x \mapsto \chi(\sigma) x, y \mapsto \psi(\sigma) y$, for some faithful characters $\chi$ and $\psi$, with $\chi^{\lambda}=\psi$.

(c) The quotient by $\mathbb{Z} / n$ of $\partial=x \frac{\partial}{\partial x}+\frac{y^{p+1}}{1+\nu y^{p}} \frac{\partial}{\partial y}$, where $p \in \mathbb{N}$, and the group acts by, $\sigma: x \mapsto \chi(\sigma) x, y \mapsto \psi(\sigma) y$, for some faithful characters $\chi$ and $\psi$, with $\psi^{p}=1$.

(d) The quotient by $\mathbb{Z} / n$ of $\partial=p x\left(1+a\left(\left(x^{q} y^{p}\right)^{d}\right)\right) \frac{\partial}{\partial x}-q y\left(1+b\left(\left(x^{q} y^{p}\right)^{d}\right)\right) \frac{\partial}{\partial y}$, where $p, q \in \mathbb{N}$ are relatively prime, $a, b$ are formal functions in one variable, the group acts by, $\sigma: x \mapsto \chi(\sigma) x, y \mapsto \psi(\sigma) y$, for some faithful characters $\chi$ and $\psi$, and $d$ is the smallest integer such that $\left(\chi^{q} \psi^{p}\right)^{d}=1$.

(e) The quotient by a dihedral type group, $G$, i.e. an extension of $\mathbb{Z} / 2$ by $\mathbb{Z} / 2 n$, where the transposition acts on $\mathbb{Z} / 2 n$ by multiplication by $p$, say, and $p^{2} \equiv 1(2 n)$. Necessarily $G$ is without pseudo-reflections. Consequently if $2^{a}$ is the maximal power of 2 dividing $2 n$ then $p \equiv \pm 1\left(2^{a}\right)$, so that if $D_{k}, \operatorname{Dih}_{k}$ denotes the binary dihedral group of order $4 k$, respectively the dihedral group of order $2 k$ (including appropriate abelian groups for small $k$ ) then either, 
$\left(\mathrm{e}^{\prime}\right) p \equiv-1\left(2^{a}\right)$, whence if we write $2 n=2^{a} l m$ for some relatively prime odd integers $l$ and $m$ with $p \equiv 1(l)$ and $p \equiv-1(m)$ then $G \stackrel{\sim}{\rightarrow} D_{2^{a-1} m} \times \mathbb{Z} / l$. In particular for a suitable choice of formal coordinates $x, y$ and $\zeta$ a $4 n$th root of unity $G$ has a representation with generators,

$$
\alpha=\left[\begin{array}{cc}
\zeta^{2} & 0 \\
0 & \zeta^{2 p}
\end{array}\right], \sigma=\left[\begin{array}{cc}
0 & \zeta^{n} \\
\zeta^{n} & 0
\end{array}\right]
$$

and acts on the foliation defined by the field,

$$
\partial=x\left(1+a\left((x y)^{l}\right)\right) \frac{\partial}{\partial x}-y\left(1+a\left(-(x y)^{l}\right) \frac{\partial}{\partial y},\right.
$$

where $a$ is a formal power series of one variable vanishing at the origin.

$\left(\mathrm{e}^{\prime \prime}\right) p \equiv 1\left(2^{a}\right), a \geq 2$, with $l, m$ as above. As such the centre of $G$ contains a unique element -1 of order 2 , and $G / \pm 1 \stackrel{\sim}{\rightarrow} \operatorname{Dih}_{m} \times \mathbb{Z} / 2^{a-1} l$. Whence $G$ could well be abelian (in fact is abelian iff $m=1, c f$. IV.4.5, since morally this corresponds to a non-classical Dynkin diagram ' $D_{3}$ '), and $G$ has no dihedral or binary dihedral subgroups. Irrespectively, we may find coordinates $x, y$ so that for $\zeta$ a $4 n$th root of unity, there is a representation with generators,

$$
\alpha=\left[\begin{array}{cc}
\zeta^{2} & 0 \\
0 & \zeta^{2 p}
\end{array}\right], \sigma=\left[\begin{array}{cc}
0 & \zeta^{m l} \\
\zeta^{m l} & 0
\end{array}\right]
$$

which, for $a$ as above, acts on the foliation defined by,

$$
\partial=x\left(1+a\left((x y)^{2^{a-1} l}\right)\right) \frac{\partial}{\partial x}-y\left(1+a\left(-(x y)^{2^{a-1} l}\right) \frac{\partial}{\partial y}\right.
$$

In particular, with the exception of (e), any $\mathbb{Q}$-foliated Gorenstein canonical singularity which is not terminal is, in fact, Gorenstein.

Unfortunately, there is no similar characterisation of log-canonical singularities. Certainly if we suppose the ambient space to have quotient singularities, then a log-canonical singularity must be as in I.2.4, albeit with $\lambda \in \mathbb{Q}_{+}$in (a). On the other hand, it is not true that the ambient space need have only quotient singularities, e.g.

Example I.2.5. On the normal surface, $x^{n+2}+y^{n+1}+x z^{n}=0, n \geq 2$, which isn't even a rational singularity, the foliation given by, $\partial=n(n+1) x \frac{\partial}{\partial x}+n(n+$ 2) $y \frac{\partial}{\partial y}+(n+1)^{2} z \frac{\partial}{\partial z}$ is log-canonical. 
Nevertheless, it's still true that the the essential difference between a logcanonical singularity (first picture) and a canonical singularity (second picture) is as per I.2.4 (a), i.e.
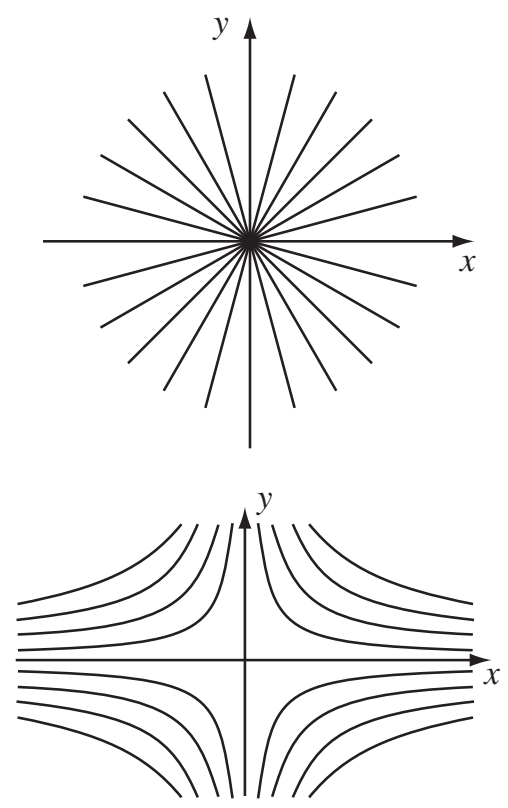

And indeed, quite generally,

Observation I.2.6. (characteristic 0) Through any $\mathbb{Q}$-foliated Gorenstein canonical singularity on a surface, there is at least one, and at most two formal invariant branches both of which are smooth.

A singularity which is neither canonical nor log-canonical is a rather different kettle of fish, and the appropriate picture would be much more like a web. In any case, the simplicity of I.2.4 should not obscure,

Warning I.2.7. Anyone unfamiliar with foliation singularities should be aware that the difference between their formal description and their analytic description is like night and day. The former is just linear algebra, the latter, even in dimension 2 can be rather tricky, e.g. all of the above types, excepting (a) under some diophantine hypothesis on $\lambda$, admit infinite dimensional spaces of analytic invariants. Nevertheless there is a sense (i.e. in a topus without enough points) in which all of these formal types converge, cf. [E1] \& [MR]. 
Apart from their simplicity, another important feature of canonical singularities on surfaces is,

Fact I.2.8. (characteristic 0) Let $\rho:(\widetilde{X}, \widetilde{\mathcal{F}}) \rightarrow(X, \mathcal{F})$ be a blow up of a foliated non-singular surface with canonical singularities then $\widetilde{\mathcal{F}}$ has canonical singularities.

Proof Indeed, in any dimension the property of being canonical is only conserved by blow ups in centres which are either invariant by the foliation or everywhere transverse to it. In dimension 2 , however, the only possible centres are points.

Consequently, given such a nice list of properties, it's helpful to know,

Fact I.2.9. (characteristic 0$)$ Let $(X, \mathcal{F})$ be a foliated algebraic surface, or even algebraic space of dimension 2 then there is a proper birational modification by a sequence of blow ups in smooth invariant centres, $\rho:(\widetilde{X}, \widetilde{\mathcal{F}}) \rightarrow(X, \mathcal{F})$ such that the induced foliation has canonical singularities.

As one might expect, the proof, [S], just involves following one's nose. The same proof works equally well in positive characteristic, but only yields logcanonical since,

Counterexample I.2.10. The foliation given by $\partial=x \frac{\partial}{\partial x}-2 y \frac{\partial}{\partial y}$ in characteristic 5 on $\mathbb{A}^{2}$ does not admit a canonical resolution by a sequence of blow ups in smooth invariant centres.

Proof. Blow up in the origin. There are two singularities, the one where the proper transform of the $y=0$ branch crosses the exceptional divisor has eigenvalues $(1,-3)$. Blow up in this then there is a singularity at the crossing of the new exceptional divisor with the proper transform of the old, and there the eigenvalues are, after multiplication by a unit, $(1,2)$. Modulo multiplication by another unit, there is a singularity on the blow up such that this new singularity has eigenvalues, $(1,-2)$, so the thing just repeats itself, and cannot be resolved.

By, I.1.9, however, this is the only thing that can go wrong, and for a logcanonical singularity which is not canonical there is obviously a weighted blow up, $\rho:(\widetilde{X}, \widetilde{\mathcal{F}}) \rightarrow(X, \mathcal{F})$ such that the induced foliation is smooth, though one should note,

Counterexample I.2.11. The foliation defined by $\partial=\frac{\partial}{\partial x}$ on $\mathbb{A}^{2}$ in character- 
istic 2 is canonical, but not terminal.

Proof Indeed, blow up once, then blow up in the induced singularity, and observe that the 2nd exceptional divisor has discrepancy 0 .

Consequently, I.2.8-9 only work in positive characteristic on replacing canonical by log-canonical, and if we want canonical then we must use the weighted blow up construction to obtain,

Fact I.2.12. (characteristic p) Let $(X, \mathcal{F})$ be a foliated algebraic surface, or even algebraic space of dimension 2 then there is a proper birational modification in the 2-category of algebraic stacks $\rho:(\widetilde{\mathcal{X}}, \widetilde{\mathcal{F}}) \rightarrow(X, \mathcal{F})$, with $\mathcal{X}$ smooth DeligneMumford, such that the induced foliation has canonical singularities.

Lest one thinks that this is just some algebraic fantasy, let's make,

Remark I.2.13. What's at stake is really a game of dimensions, and this game plays out very differently in dimension 3. Indeed, I.2.9 is false in characteristic zero, and dimension 3, while I.2.12 remains true, cf. [M7] I.1.

The final thing to note in dimension 2, is that by way of Mumford's intersection theory we can generalise the definition of discrepancy, and whence those of canonical and terminal singularities, to arbitrary foliations on normal algebraic spaces irrespective of whether they are $\mathbb{Q}$-foliated Gorenstein or not. Indeed, we have Mumford's Néron-Severi group $\operatorname{NS}^{m}(X)$, given by,

Definition I.2.14. Let $X$ be a proper 2-dimensional algebraic space and $p: Y \rightarrow$ $X$ a relatively projective birational morphism from a smooth projective variety $Y$ (such a $Y$ always exists by $[\mathrm{Kn}]$ ) and $E_{i}$ the curves on $Y$ contracted by $p$ then for $C$ a curve on $X$ with $\widetilde{C}$ a curve on $Y$ there exist unique positive rational numbers $a_{i}$ (by virtue of the fact that the intersection matrix $E_{i} \cdot E_{j}$ is negative) such that if $p^{*} C:=\widetilde{C}+\sum_{i} a_{i} E_{i}$ then, $p^{*} C \cdot E_{j}=0$ for any $j$. We may then define for two curves $C$ and $C^{\prime}$, an intersection product $C \cdot C^{\prime}:=p^{*} C \cdot p^{*} C^{\prime}$, and $\operatorname{NS}^{m}(X)$ is the free $\mathbb{Q}$-vector space of curves in $X$ modulo numerical equivalence in this sense. The group $\mathrm{NS}^{m}(X)$ coincides with the group $\mathrm{NS}(X)$ in the usual sense iff $X$ has $\mathbb{Q}$-factorial singularities.

Consequently with this definition in mind for $(X, \mathcal{F})$ a 2-dimensional foliated algebraic space, $K_{\mathcal{F}}$ always has sense in $\mathrm{NS}^{m}(X)$ and we may extend definition 
I.1.2, and the notions of being canonical and terminal to any proper 2-dimensional foliated algebraic space. In this respect one notes,

Fact I.2.15. $(X, \mathcal{F})$ as above, $E$ an exceptional divisor in $k(X)$ then $a_{X, \mathcal{F}}(E)$ is well-defined, and coincides with the previous definition when the foliation is $\mathbb{Q}$-Gorenstein.

There is, however, in the extended sense one non $\mathbb{Q}$-Gorenstein singularity which we'll encounter in III.0.3. A particularly appealing model for it in line with I.2.1-4 is as the quotient by $\mathbb{Z}$ of a neighbourhood of infinity on a bi-disc, with the foliation being either of the projections. This 'new' singularity is almost single handedly responsible for many of the distinctions between classical surface classification, and the current discussion.

\section{I.3. Residues}

This is a subject of some interest in its own right, and is the only point at which we even vaguely consider a question which may properly be considered as dynamical. Indeed residues arise in the context of the Leray spectral sequences for $X \rightarrow[X / \mathcal{F}]$, with the presence of singularities giving rise to different spectral sequences depending on whether we use projective resolutions (smooth forms) or injective resolutions (distributions), and the difference between these spectral sequences at the $E_{2}$ term is the residue. This is discussed at length in [M7] IV.1-2, but our present considerations are rather more mundane. Indeed, in the course of doing canonical model theory, there will arise certain configurations of invariant algebraic curves which we wish to exclude. The most informative way to exclude these configurations is by calculating the holonomy, in relation to which the residue calculus that will be employed is a rather poor first order and numerical vestige. Nevertheless, it will keep everything within the framework of the sort of numerical argument typical in the theory of algebraic surfaces. For ease of exposition we'll work with varieties $(X, \mathcal{F})$ foliated by curves over a field $k$, with underlying space $X$ non-singular. We consider the foliation as given by a short exact sequence,

$$
0 \longrightarrow \mathcal{N} \longrightarrow \Omega_{X} \longrightarrow K_{\mathcal{F}} \cdot I_{Z} \longrightarrow 0
$$

where $I_{Z}$ is the ideal of the singular subscheme of $\mathcal{F}$ and $\mathcal{N}$ is a saturated subsheaf 
of $\Omega_{X}$. In dimension $2, \mathcal{N}$ will be a line bundle.

Firstly let's consider the $(1,1)$ part of the cohomology. What interests us is $N^{1}:=\operatorname{Ker}\left\{H^{1,1} \rightarrow H^{1}\left(K_{\mathcal{F}}\right)\right\}$, which at the $(n-1, n-1)$ level is dual to $N_{1}:=$ $\operatorname{Im}\left\{\operatorname{Ext}^{n-1}\left(K_{\mathcal{F}} \cdot I_{Z}, K_{X}\right) \rightarrow H^{n-1, n-1}\right\}$.

Now suppose $f: C \rightarrow X$ is an invariant curve with $C$ smooth, then by the definition of invariance, $f^{*} \mathcal{N}$ maps to zero in $\omega_{C}$, i.e. $[f] \in N_{1}$. The essential then of the Residue calculus that will be employed is that if $D$ is a class in $N^{1}$ the pairing induced by $H^{1,1}$ with $H^{n-1, n-1}$ factors as,

$$
N^{1} \otimes N_{1} \longrightarrow H^{0}\left(K_{\mathcal{F}} \otimes \mathcal{O}_{Z}\right) \otimes \operatorname{Ext}^{n}\left(K_{\mathcal{F}} \otimes \mathcal{O}_{Z}, K_{X}\right) \longrightarrow k
$$

For $Z$ of dimension 0, there isn't much difference between local and global duality, and the traditional interpretation in terms of the residue symbol à la [BB] follows from [Gro] IV.5.4. This is however, fairly unimportant. What interests us are what are the elements of $N^{1}$, and when is the pairing over determined. One obvious class of elements in $N^{1}$ is invariant divisors, i.e. an effective divisor $\mathcal{O}_{X}(D)$ such that if locally it is given by $f_{\alpha}=0$, and $\partial_{a}$ defines the foliation, then $\partial_{\alpha}\left(f_{\alpha}\right) \in\left(f_{\alpha}\right)$, so that $\frac{\partial_{\alpha} f_{\alpha}}{f_{\alpha}} \otimes \partial_{\alpha}^{\vee}-\frac{\partial_{\beta} f_{\beta}}{f_{\beta}} \otimes \partial_{\beta}^{\vee}$ is a co-boundary for the chern class of $D$ along $\mathcal{F}$. The other important example is the chern class of $\mathcal{N}$. Since $H^{1}\left(X, K_{\mathcal{F}}\right)$ injects into $H^{1}\left(X \backslash Z, K_{\mathcal{F}}\right)$, we can just check this over the smooth locus. For an affine patch let $\omega_{1}^{\alpha}, \ldots, \omega_{n}^{\alpha}$ be a basis of $\mathcal{N}$, and $\tau^{\alpha}$ a lifting of $\partial_{\alpha}^{\vee}$, then we have for units $h_{\alpha \beta}$,

$$
\omega_{1}^{\alpha} \wedge \ldots \wedge \omega_{n}^{\alpha}=h_{\alpha \beta} \omega_{1}^{\beta} \wedge \ldots \wedge \omega_{n}^{\beta} .
$$

On the other hand, $d \omega_{i}^{\alpha}=a_{i j}^{\alpha} \tau^{\alpha} \wedge \omega_{j}^{\alpha}(\bmod \mathcal{N} \wedge \mathcal{N})$ for functions $a_{i j}$, with summation convention. Whence on differentiating we have,

$$
\begin{gathered}
(-1)^{i-1}\left(a_{i i}^{\alpha}\right) \tau^{\alpha} \wedge \omega_{1}^{\alpha} \wedge \ldots \wedge \omega_{n}^{\alpha}= \\
d h_{\alpha \beta} \omega_{1}^{\beta} \wedge \ldots \wedge \omega_{n}^{\beta}+h_{\alpha \beta}(-1)^{i-1} a_{i i}^{\beta} \tau^{\beta} \wedge \omega_{1}^{\beta} \wedge \ldots \wedge \omega_{n}^{\beta} .
\end{gathered}
$$

cancelling the $\omega_{1}^{\beta} \wedge \ldots \wedge \omega_{n}^{\beta}$ and restricting to $K_{\mathcal{F}}$, we find,

$$
\frac{\partial h_{\alpha \beta}}{h_{\alpha \beta}} \otimes \partial^{\vee}=(-1)^{i-1} a_{i i}^{\alpha} \partial_{\alpha}^{\vee}-(-1)^{i-1} a_{i i}^{\beta} \partial_{\beta}^{\vee}
$$


i.e. the chern class restricts trivially in cohomology as claimed. For amusement therefore we can note the kind of formulae which one obtains.

Example I.3.1. Let $f: C \rightarrow X$ be the normalisation of an invariant curve of a foliation by curves $\mathcal{F}$ on $X$ over a field $k$. Suppose $f(C) \not \subset \operatorname{sing}(\mathcal{F})$, and for a point $c \in f^{-1}(\operatorname{sing} \mathcal{F})$ let $x_{1}, \ldots, x_{n}$ be coordinates in the completion of the local ring at $f(c)$, with the foliation being given by a field $\partial=a_{i} \frac{\partial}{\partial x_{i}}, a_{i} \in \mathfrak{m}_{f(c)}$, summation convention. Define, $\operatorname{Tr}_{f(c)}(\partial):=\frac{\partial a_{i}}{\partial x_{i}}$, and let $\mathcal{N}_{\mathcal{F}}$ be the normal bundle to the foliation then,

$$
c_{1}\left(\mathcal{N}_{\mathcal{F}}\right) \cdot C=\sum_{c \in f^{-1}(\operatorname{sing} \mathcal{F})} \operatorname{Res}_{c}\left(\frac{f_{*}\left(\frac{\partial}{\partial \xi}\right)}{f^{*} \partial} f^{*} \operatorname{Tr}_{f(c)}(\partial) d \xi\right)
$$

where $\xi$ is a coordinate in $\widehat{\mathcal{O}}_{C, c}$, and the residue is taken in the sense of Grothendieck (= ordinary sense in characteristic zero) in the field $k$.

However, as we've already said, it's primarily over determination rather than explicit formulae which interests us. Over a surface, everything is quite nice. Indeed canonically $N^{1}=N_{1}$, and we suppose that we have a collection of invariant curves $C_{i}$ such that the intersection matrix $C_{i} \cdot C_{j}$ is invertible in $k$. Each curve $C_{i}$ defines a class in $N^{1}$ and we have for each connected component $z$ contained in $\underset{i}{\cup} C_{i}$ of the singular locus of $\mathcal{F}$ understood with reduced structure, residue maps,

$$
\operatorname{Res}_{z}: N^{1} \longrightarrow K_{\mathcal{F}} \otimes \mathcal{O}_{z} \stackrel{\sim}{\longrightarrow} k[z]
$$

We put $\sum_{z} \operatorname{dim}_{k} k[z]=\operatorname{deg}\left(\operatorname{sing}(\mathcal{F}) \cap \bigcup_{i} C_{i}\right)=d$, say, and observe that if $d<$ number of curves $C_{i}$, then there are elements $\lambda_{i}$ of $k$, not all zero, such that, $\sum_{i} \lambda_{i} C_{i}$ has no residue, and so lies in,

$$
\operatorname{Im}\left\{H^{1}(X, \mathcal{N}) \longrightarrow H^{1,1}\right\}
$$

Consequently we must have $\left(\sum_{i} \lambda_{i} C_{i}\right) \cdot C_{j}=0$ for any $j$. This however contradicts the invertibility of the intersection matrix, and so we have proved,

Fact I.3.2. Notations as above if the intersection matrix $C_{i} \cdot C_{j}$ is invertible in 
$k$ (e.g. $\cup_{i} C_{i}$ contractible, and characteristic zero) then,

The number of curves $C_{i} \leq \operatorname{deg}\left(\operatorname{sing}(\mathcal{F}) \cap \cup_{i} C_{i}\right)$.

\section{Complements to Ekedahl, Miyaoka et al.}

\section{II.1. $p$-closure}

T. Ekedahl, [E], and independently Rudakov \& Shafarevich, [RS], discovered that foliations in positive characteristic admit a remarkably rich structure which under mild hypothesis renders them amenable to a highly algebraic interpretation. The important new feature which renders this possible is the $p^{\text {th }}$-power map, i.e. one easily verifies that if $\partial$ is a derivation in positive characteristic then $\partial^{p}$ is again a derivation. In particular with notations as in I.1, if $(X, \mathcal{F})$ is a foliation then there is a map by $p^{\text {th }}$-powers,

$$
\mathcal{T}_{\mathcal{F}_{0}}^{p} \rightarrow \mathcal{T}_{X_{0}} / \mathcal{T}_{\mathcal{F}_{0}}
$$

and we call the foliation $p$-closed if this map is zero. One then has,

Proposition II.1.1. [E] There is a one to one correspondence between,

(a) $p$-closed foliations with rank of $\mathcal{T}_{\mathcal{F}}=r$.

(b) Factorisations $X \underset{\rho}{\longrightarrow} Y \underset{\sigma}{\longrightarrow} X^{(1)}$ of (geometric) Frobenius, $\operatorname{deg}(\rho)=p^{r}$.

In this correspondence, given a foliation one obtains $Y$ from the ring of functions $\left\{f \in \mathcal{O}_{X} \mid \partial(f)=0, \partial \in \mathcal{T}_{\mathcal{F}}\right\}$, while given a factorisation $\mathcal{T}_{\mathcal{F}}$ is just the kernel of $\rho_{*}$. Should $\mathcal{T}_{\mathcal{F}}$ be $p$-closed, we naturally write $X / \mathcal{F}$ for the quotient $Y$. There is however some need for caution regarding $X / \mathcal{F}$ even if $X$ is nonsingular. Specifically denote by $\operatorname{sing}(\mathcal{F})$ the loci where either $X$ is singular or $\mathcal{F}$ is not locally free, then in general $X / \mathcal{F}$ is highly singular on $\rho(\operatorname{sing}(\mathcal{F}))$. Certainly an easy calculation shows that $X / \mathcal{F}$ is normal but it certainly need not be Gorenstein, although we do have:

Proposition II.1.2. [E] If $X$ is $\mathbb{Q}$-factorial then $X / \mathcal{F}$ is $\mathbb{Q}$-factorial, and:

$$
\rho^{*} K_{X / \mathcal{F}}=(p-1) K_{\mathcal{F}}+K_{X} .
$$


A representative example of the kind of singularity that one encounters on $X / \mathcal{F}$ comes from the foliation given by $\partial=x \frac{\partial}{\partial x}+y \frac{\partial}{\partial y}$ in characteristic $p$, so $\mathcal{O}_{Y}=k\left[x^{p}, x^{p-1} y, \ldots, x y^{p-1}, y^{p}\right]$, and $Y$ is the cone over a rational normal curve, which, incidentally, is an étale quotient singularity even in char $=p$. That up to minor trivialities this is indeed the general case follows from,

Proposition II.1.3. Let $\left(X_{0}, \mathcal{F}_{0}\right)$ be a foliation in characteristic zero with canonical singularities and $(X, \mathcal{F}) \rightarrow$ Spec $\mathcal{O}$ a model over an affine open subset of a finitely generated extension of $\mathbb{Z}$ such that for all maximal ideals $\mathfrak{m}$ of $\mathcal{O}$ outside a Zariski closed subset, $\left(X_{\mathfrak{m}}, F_{\mathfrak{m}}\right)$ is $p=\operatorname{char} k(\mathfrak{m})$ closed, then at any point $z \in \operatorname{sing}\left(\mathcal{F}_{0}\right)$ we can find formal coordinates $x_{1}, \ldots, x_{n}, n \leq \operatorname{dim} X$, and rational integers $\lambda_{i}$ such that the foliation is given by a field of the form,

$$
\partial=\sum_{i=1}^{n} \lambda_{i} x_{i} \frac{\partial}{\partial x_{i}} \in \operatorname{Der}\left(\widehat{\mathcal{O}}_{X_{0}, z}\right) .
$$

Proof. We use our Jordan decomposition. Indeed quite generally let $\partial$ be a derivation of a regular local ring $\widehat{\mathcal{O}}$ over a field $k$ of positive characteristic. We can write down a Jordan decomposition $\partial=\partial_{S}+\partial_{N}$, of $\partial$ into semi-simple and nilpotent parts. An identity of Jacobson, [E] 4.2, gives:

$$
\partial^{p}=\partial_{S}^{p}+\partial_{N}^{p}+\sum S_{k}\left(\partial_{S}, \partial_{N}\right)=\partial_{S}^{p}+\partial_{N}^{p}
$$

where the last identity follows from the fact that the $S_{k}$ are Lie polynomials, and $\left[\partial_{S}, \partial_{N}\right]=0$. However if the foliation is $p$-closed then $\partial^{p} \wedge \partial=0$, so:

$$
0=\partial_{S} \wedge \partial_{S}^{p}+\partial_{S} \wedge \partial_{N}^{p}+\partial_{N} \wedge \partial_{S}^{p}+\partial_{N} \wedge \partial_{N}^{p}
$$

Typically if $\mathfrak{m}$ is the maximal ideal of $\widehat{\mathcal{O}}, \partial_{N}(\mathfrak{m}) \subset \mathfrak{m}^{2}$, so: $\partial_{N}^{p}(\mathfrak{m}) \subset \mathfrak{m}^{p+1}$. However $p$ could be close to the dimension etc., so we impose the hypothesis that our foliation is specialised from characteristic zero. In this case we're studying the completion $\widehat{\mathcal{O}}$ and a derivation $\partial$ of it and we already know the Jordan decomposition of $\partial \bmod \mathfrak{m}^{d}$, say, then for $p$ sufficiently large this is the same as that for the specialisation. Bearing this in mind and that $\partial_{S} \neq 0$ if the singularities are canonical we take suitable coordinates in characteristic zero such that,

$$
\partial_{S}=\lambda_{i} x_{i} \frac{\partial}{\partial x_{i}} \quad \text { and } \quad \partial_{N}(\text { if it exists })=f_{i} \frac{\partial}{\partial x_{i}},\left(f_{1}, \ldots, f_{n}\right) \not \equiv 0\left(\bmod \mathfrak{m}^{d}\right)
$$


where $\mathfrak{m}^{d}$ is some suitable power of the maximal ideal. Certainly for some $e$, independent of $p, \partial_{N}^{e}(\mathfrak{m}) \subset \mathfrak{m}^{2}$, so for $p$ sufficiently large to avoid bad reduction, $\partial_{N}(\mathfrak{m}) \subset \mathfrak{m}^{\left[\frac{p}{e}\right]}$. As ever for $p$ large, we can look at $(*) \bmod \mathfrak{m}^{3}$, and begin by supposing $\partial$ is not semi-simple $\bmod \mathfrak{m}^{2}$. In particular we have a Jordan block of the form

$$
D=\sum_{i=1}^{m} \lambda x_{i} \frac{\partial}{\partial x_{i}}+\sum_{i=k}^{m} x_{i-1} \frac{\partial}{\partial x_{i}}
$$

for some sub-system of coordinates $x_{1}, \ldots, x_{m}, m \geq 2$, and all the $\frac{\partial}{\partial x_{i}} \wedge \frac{\partial}{\partial x_{j}}$ terms $i, j \leq m$ occurring in $* \bmod \mathfrak{m}^{2}$ arise from $D$. Plainly, therefore, $\lambda=0$. However there is a non-zero eigenfunction $x_{m+1}$, say, so the nilpotent part is also zero, as claimed. Continuing to work $\bmod \mathrm{m}^{2}$, given we have no nilpotent part, the eigenvalues considered as a point in projective space must be stable under Frobenius, and so certainly in $\mathbb{F}_{p}$. Given this for almost all $p$, the eigenvalues, modulo multiplication by a suitable unit, are indeed rational integers. Making such a multiplication we have $\partial_{S}^{p}=\partial_{S}$ and $\partial_{N} \wedge \partial_{S}=0\left(\bmod \mathfrak{m}^{d+1}\right)$ for almost all $p$, so in $\mathcal{O} / \mathfrak{m}^{d} \partial, \partial_{N}$ is parallel to $\partial_{S}$. Since $d$ was arbitrary, this proves the proposition after multiplication by a suitable formal unit.

Having singularities which are formally linearisable with rational eigenvalues is already a rather strong property, but we can easily go further, for example:

Divertimento II.1.4. Let $\left(X_{0}, \mathcal{F}_{0}\right)$ be a foliated non-singular surface in characteristic zero such that in the notations of II.1.3, $\left(X_{\mathfrak{m}}, \mathcal{F}_{\mathfrak{m}}\right)$ is $p$-closed for all $\mathfrak{m}$ outside a Zariski closed set, then $\left(X_{0}, \mathcal{F}_{0}\right)$ is given by an infinitesimal formal flat groupoid.

Proof. The issue here is the flatness. Let $\Delta$ be the diagonal in $X \times X$, and $\mathcal{P}$ the completion of the latter in the former. Away from the singularities the groupoid is just the formal scheme $\mathcal{F}$, say, obtained by adding in a germ of the foliation in the direction of the second factor. If we can complete $\mathcal{F}$ over the singularities then flatness follows. Whence without loss of generality $X$ is affine, and we're only worried about an isolated singularity $z$. Now let's say the foliation is given by a vector field $\partial$ on $X$, and $x, y$ are generators of $\mathfrak{m}$ at $z$. We can make affine patches $U_{x}, U_{y}$ of $X^{*}=X \backslash z$ defined as $\partial(x) \neq 0$ and $\partial(y) \neq 0$ respectively. Over $\mathcal{P}_{u_{x}}$ and $\mathcal{P}_{u_{y}}$ (fibres via the $1^{\text {st }}$ projection) the groupoid is cut out by divisors $D_{x}$ and $D_{y}$, which a priori do not patch. The problem is that $\mathcal{P}_{X^{*}}$ has an enormous 
local Picard group. Call $D$ the class so defined in $\operatorname{Pic}\left(\mathcal{P}_{X^{*}}\right)$. Being in dimension 2 , if $\mathcal{P}_{n}$ is the $n^{\text {th }}$-thickening we have an exact sequence,

$$
0 \rightarrow H^{1}\left(X^{*}, S^{n} \Omega_{X}\right) \rightarrow \operatorname{Pic}\left(\mathcal{P}_{n+1} \otimes \mathcal{O}_{X^{*}}\right) \rightarrow \operatorname{Pic}\left(\mathcal{P}_{n} \otimes \mathcal{O}_{X^{*}}\right) \rightarrow 0 .
$$

So we need $D$ to be zero in each $\operatorname{Pic}\left(\mathcal{P}_{n} \otimes \mathcal{O}_{X^{*}}\right)$. However for fixed $n$, and $p$ sufficiently large the obstruction $D$ just specialises to the obstruction to completing the groupoid to order $n$ in characteristic $p$. However, if we have $p$-closure and $n<p$ this is trivial - just take the Zariski closure of the inseparable groupoid over the singularities. Having patched $D_{x}, D_{y}$ at the bundle level we now have $\mathcal{F}$ over $X^{*}$ defined as the zero locus of a formal function $f$ on $\mathcal{P}_{X^{*}}$, and general local cohomology nonsense extends $f$ to all of $\mathcal{P}_{X}$, as required.

A couple of remarks are probably in order,

Remark II.1.5. (a) The proof of II.1.4 works equally well for codimension 1 foliations in general. For non-codimension 1, flatness would require more special singularities, however it ought still to be true that $p$-closure for almost all $p$ forces the completion of the groupoid in characteristic zero. Indeed, morally (modulo the flatness) II.1.3 is equivalent to completing the groupoid, but without some analytic convergence, it seems just a bit weaker.

(b) Even $p$-closure at infinitely many primes is already a strong condition. Indeed II.1.4 goes through verbatim to show that under this hypothesis the field $\partial$ is semisimple. In addition, and rather evidently, $p$-closure can only take place when the eigenvalues considered as a point of projective space take values in $\mathbb{F}_{p}$.

Certainly, almost by definition, $p$-closure is true globally iff it is true formally at one point. Using the theory of Jordan decomposition we can elucidate this somewhat by way of a characteristic $p$ Frobenius theorem,

Divertimento II.1.6. Let $\partial$ be a non-singular derivation of a complete regular local ring $A$ over an algebraically closed field $L$ of characteristic $p>0$ isomorphic to its residue field then there is a choice of coordinates $x, y_{1}, \ldots, y_{n}$ in the maximal ideal such that up to multiplication by a unit,

$$
\partial=\frac{\partial}{\partial x}+\sum_{i=1}^{n} x^{p-1} f_{i}\left(x^{p}, y\right) \frac{\partial}{\partial y_{i}} .
$$

Proof. We can certainly multiply $\partial$ by a unit, in such a way that for some $x \in \mathfrak{m}$, 
$\partial x=1$. Now consider $\widetilde{\partial}=x \partial$, and its Jordan decomposition $\partial_{S} \oplus \partial_{N}$. Trivially $\partial_{S}=x \frac{\partial}{\partial x}$, in some coordinate system $x, y_{1}, \ldots, y_{n}$. Observe that in our formulae for the nilpotent part we must have an exponent of $x$ in the monomial $\left(x^{Q} x_{i}\right)$ at least 1 , since $x \mid \widetilde{\partial}$, whence the claim.

This is true for any foliation (and incidentally implies the Frobenius theorem in characteristic zero) and the subscheme cut out by the vanishing of the $p$-curvature is that defined by the ideal $\left(f_{1}, \ldots, f_{n}\right)$. In particular it is invariant by the foliation (although this hardly requires special coordinates $\partial\left(\partial^{p} y_{i}\right)=$ $\partial^{p}\left(\partial y_{i}\right)=\partial^{p}\left(y_{j}\right) \frac{\partial}{\partial y_{j}}\left(\partial y_{i}\right)$, given $\partial x=1$ will do $)$. One should be careful however since the reduction of this subscheme may not be invariant, e.g. $f_{i}\left(x^{p}, y\right)=x^{p}$. In any case, the important point, is that the invariant subscheme defined by the singular locus even when the corresponding reduced subscheme is invariant can more or less be arbitrarily complicated and certainly does not lift to characteristic zero. Indeed, for,

$$
\partial=\frac{\partial}{\partial x}+x^{p-1}\left(y^{2}+x^{p}\right) \frac{\partial}{\partial y}
$$

$y^{2}+x^{p}=0$ is an invariant curve, which isn't even smooth though the foliation (which is not $p$ closed) is.

\section{II.2. Conditions for $p$-closure}

Truly satisfactory conditions in high dimension require a lot of work in analysing the structure of the subscheme where the $p$-closure vanishes, so we'll restrict ourselves here to dimension 2, with underlying space $X$ non-singular. We'll use the notations of II.1.3, i.e. $\left(X_{0}, \mathcal{F}_{0}\right)$ is a foliation in characteristic zero, and $(X, \mathcal{F}) \rightarrow \operatorname{Spec} \mathcal{O}$ a model over an affine open subset of a finitely generated extension of $\mathbb{Z}$ with $\left(X_{\mathfrak{m}}, \mathcal{F}_{\mathfrak{m}}\right)$ the reduction at a maximal ideal. The most obvious condition is,

Lemma II.2.1., [S-B], Let $D_{0}$ be a nef. divisor on $X_{0}$ with $K_{\mathcal{F}_{0}} \cdot D_{0}<0$ then for $p$ sufficiently large and $\operatorname{char} k(\mathfrak{m})=p,\left(X_{\mathfrak{m}}, \mathcal{F}_{\mathfrak{m}}\right)$ is $p$-closed.

Of which, an essentially optimal singular variant is,

Lemma II.2.2. Notations as above, and suppose $z \in \operatorname{sing}(\mathcal{F})$ is a canonical singularity without nilpotent structure and irrational eigenvalue $\lambda$, with $\varepsilon<$ $\min \left\{\frac{1}{H(\lambda)}, 1\right\}$ where $H(\lambda)$ is the big height of $\lambda$ in the Fubini-Study metric, then 
if $D_{0}$ is effective with its proper transform nef. and $K_{\mathcal{F}_{0}} \cdot D_{0}<\varepsilon \operatorname{ord}_{z}\left(D_{0}\right)$, $\left(X_{\mathfrak{m}}, \mathcal{F}_{\mathfrak{m}}\right)$ is p-closed for all maximal ideals $\mathfrak{m}$ of $\mathcal{O}$ such that $p$ splits completely and is sufficiently large.

Proof. What's at stake is to ask how large is the order of vanishing of the $p$ closure map $T_{\mathcal{F}}^{p} \rightarrow N_{\mathcal{F}} \cdot I_{Z}$ around $p$, where $N_{\mathcal{F}}$ is the normal bundle to the foliation. We make use of I.2.3, and let $\partial$ be a local generator of $T_{\mathcal{F}}$ about $z$ with $\partial_{S} \oplus \partial_{N}$ a Jordan decomposition. Necessarily for $p$ sufficiently large, we have $\partial_{S}=x \frac{\partial}{\partial x}+\lambda y \frac{\partial}{\partial y}$ for some suitable coordinates $x, y$ in $\widehat{\mathcal{O}}_{X, z}$, and as ever,

$$
\partial^{p}=\partial_{S}^{p}+\partial_{N}^{p}=\partial_{S}+\partial_{N}^{p}
$$

so the order of vanishing of the $p$-closure map is the order of vanishing of, $\partial_{S} \wedge$ $\partial_{N}^{p}+\partial_{N} \wedge \partial_{S}+\partial_{N} \wedge \partial_{N}^{p}$. Now $\partial_{N}^{p} \wedge \partial$ vanishes at least to order $p$ unless $\lambda=1$ in $k(\mathfrak{m})$ which given it's irrationality we might as well say doesn't happen by taking $p$ sufficiently large.

Consequently we're reduced to looking at $\partial_{N} \wedge \partial_{S}$. The nilpotent part is given by the conditions of I.2.3 which are of the form $q_{1} \lambda+q_{2}=0$ for $q_{i} \in \mathbb{N} \cup\{0\} \cup\{-1\}$, and the nilpotent part vanishes to order at least $q_{1}+q_{2}+1$. Consequently if in standard coordinates $[S, T]$, say, on $\mathbb{P}^{1}$ we identify $\lambda$ with $[\lambda, 1]$ and $Q$ with the section $\gamma_{Q}=q_{1} S+q_{2} T$ of the tautological bundle then our condition becomes $\gamma_{Q}(\lambda)=0$, and the order of vanishing is at least the big height of $\gamma_{Q}$ in the Fubini-Study metric. Even better the pairing $(Q, \lambda) \mapsto \gamma_{Q}(\lambda)$ just corresponds to intersecting $\lambda \times Q$ with respect to the diagonal on $\mathbb{P}^{1} \times \mathbb{P}^{1}$ in the Arakelov sense, and $(\mathcal{O}: Z)\{h(\lambda)+h(Q)\}$ equals,

$$
\sum_{\mathfrak{m}} \operatorname{ord}_{\mathfrak{m}}\left(\gamma_{Q}(\lambda)\right) \log (\# k(\mathfrak{m}))-\sum_{k(\mathcal{O}) \underset{\sigma}{\hookrightarrow} \mathbb{C}} \frac{1}{2} \log \left(\frac{\left|q_{1} \lambda^{\sigma}+q_{2}\right|^{2}}{\left(\left|q_{1}\right|^{2}+\left|q_{2}\right|^{2}\right)\left(1+\left|\lambda^{\sigma}\right|^{2}\right)}\right)
$$

Since $\lambda$ is irrational, $\Lambda \cdot Q$ is not identically zero, whence if $\lambda q_{1}+q_{2}=0$ and $p$ splits completely, $p \leq H(\lambda) H(Q)$, which tells us that the nilpotent part vanishes to order at least $p / H(\lambda)$, so that in turn the $p$-curvature at $z$ vanishes to order at least $p \min \left\{1, H(\lambda)^{-1}\right\}>p \varepsilon$. Possibly shrinking $\varepsilon$ a little, we may assume $\widetilde{D}_{\mathfrak{m}}$ is ample, and as $\mathbb{Q}$ divisors $p K_{\mathcal{F}}+N_{\mathcal{F}}-p \min \left\{1, H(\lambda)^{-1}\right\} E_{z}, E_{z}$ being the exceptional divisor over $z$, is effective which enjoys the absurdity of intersecting $\widetilde{D}_{\mathfrak{m}}$ negatively for $p$ sufficiently large. $\square$ 
Obviously the condition of $p$ being totally split wasn't really necessary, the weaker condition $k(\mathfrak{m})=\mathbb{F}_{p}$ would have worked equally well provided we took the worse bound of, $\min \left\{\frac{1}{(\mathcal{O}: Z) H(\lambda)}, 1\right\}$. What is essential in the proof is that $\lambda$ is irrational. One might hope that rational is easier to treat, which is certainly true if the singularity were linearisable in characteristic zero, then we could actually get the lemma for $\varepsilon=1-\delta$, any $\delta>0$, but if it isn't the $p$-closure map will only have a finite order at $z$ independent of $p$, and the lemma appears hopeless. Nevertheless the lemma casts a lot of light on some examples discovered by Ekedhal, ShepherdBaron, and Taylor, [EST], of which the most interesting from our point of view is,

Example II.2.3. (Hilbert Modular Surfaces) Let $\mathcal{O}$ be the ring of integers in a real quadratic number field. The group $\Gamma=\mathrm{SL}_{2}(\mathcal{O})$ acts on two copies of the upper half plane $\mathfrak{H} \times \mathfrak{H}$ by way of its two natural embeddings in $\mathrm{SL}_{2}(\mathbb{R})$. Modulo \pm 1 , the action is properly discontinuous and the resulting surface $\mathfrak{H} \times \mathfrak{H} / \Gamma$ is quasi-projective (Hilbert-Modular forms give the quasi-projective embedding) with at worst quotient singularities. Furthermore we can complete things to a projective surface $S_{0}$ by adding in finitely many cusps which are elliptic Gorenstein singularities resolved by elliptic polygons or a rational curve with a node. The surface $S_{0}$ is called the Baily-Borel compactification, and its minimal desingularisation $\rho: S \rightarrow S_{0}$ is what is usually termed a Hilbert-Modular surface. The foliations determined by the splitting descend to foliations $\mathcal{F}_{0}, \mathcal{G}_{0}$ on $S_{0}$. Neither is $\mathbb{Q}$-foliated Gorenstein in the presence of cusps, cf. IV.2.2, but they do have canonical singularities in the sense of I.2.14. There is a splitting $\mathcal{T}_{S_{0}}=\mathcal{T}_{\mathcal{F}_{0}} \oplus \mathcal{T}_{\mathcal{G}_{0}}$, and even better if $D$ is the reduced simple normal crossing divisor contracted by $\rho$ then it is invariant by the induced foliations $\mathcal{F}$ and $\mathcal{G}$, and both of these not only have canonical singularities but, for simplicity, in the absence of quotient singularities,

$$
\Omega_{S}^{1}(\log D)=K_{\mathcal{F}} \oplus K_{\mathcal{G}}
$$

In particular $K_{\mathcal{F}}, K_{\mathcal{G}}$ are pseudo-effective divisors of numerical Kodaira dimension 1 and Kodaira dimension $-\infty$ (essentially because any section would force the foliation to be a fibration, cf. IV.5.1) so that for any $\varepsilon>0$ there is an ample divisor $H_{\varepsilon}$ on the blow up of $X$ in any singularity $z$, say, of $\mathcal{F}$ such that $K_{\mathcal{F}} \cdot H_{\varepsilon}<$ $\varepsilon \operatorname{ord}_{z}\left(H_{\varepsilon}\right)$ (otherwise $K_{\mathcal{F}}$ would have a section cf. IV.5.7). Now consider the 
case where $z$ lies on a polygon. The singularities are exactly the crossings of the elliptic polygon, and within a polygon the eigenvalue at any one singularity can be calculated in terms of any other via the residue-formula I.3.1. Actually we're in the degenerate case of I.3.2 where the number of curves is exactly the number of singularities, and the eigenvalues may be computed as continued fractions in the intersection matrix, cf. IV.2.2. In any case the eigenvalues are thus in one to one correspondence with the non-principal ideal classes of $\mathcal{O}$, and are certainly irrational provided $S_{0}$ has cusps. Whence combining II.2.2 with II.1.3 we see that for $p$ sufficiently large, $(X, \mathcal{F})$ is $p$-closed iff $p$ splits completely in $\mathcal{O}$. In particular, [EST], $p$ closure for infinitely many $p$ does not imply algebraicity of the leaves in characteristic zero.

\section{II.3. Adjunction}

We begin by defining the Segre class along the foliation's singular subscheme,

Definition II.3.1. Let $\Omega_{X} \rightarrow K_{\mathcal{F}} \cdot I_{Z} \rightarrow 0$ be a foliation by curves on a nonsingular variety $X$, and $\widetilde{X}$ in $\pi: \mathbb{P}\left(\Omega_{X}\right) \rightarrow X$ the closure of the corresponding section over the smooth locus, then $\widetilde{X} \stackrel{\sim}{\rightarrow} \mathrm{Bl}_{Z}(X)$ (understood with nilpotent structure) and if $E$ is the exceptional divisor and $L$ the tautological bundle then $L=\pi^{*} K_{\mathcal{F}}(-E)$ and for $C$ in $X$ a curve we define the Segre class $s_{Z}(C)$ of $Z$ along $C$ to be $E \cdot \widetilde{C}$ where $\widetilde{C}$ is the proper transform.

Now adjunction for $(X, \mathcal{F})$ a foliation by curves on a non-singular surface takes two forms either:

(a) $C$ is a curve not invariant by the foliation and we have natural maps,

$$
\mathcal{O}_{C}(-C) \rightarrow \Omega_{X} \otimes \mathcal{O}_{C} \rightarrow K_{\mathcal{F}} \otimes \mathcal{O}_{C}
$$

so $\left(K_{\mathcal{F}}+C\right) \cdot C \geq 0$. Whence, $K_{\mathcal{F}} \cdot C<0 \Rightarrow C^{2}>0$, i.e. $C$ is big and nef.

(b) $C$ is invariant by the foliation, so for $\nu: \widetilde{C} \rightarrow C$ its normalisation,

$$
K_{\mathcal{F}} \cdot C=(2 g(\widetilde{C})-2)+s_{Z}(C)-\operatorname{Ram}_{\widetilde{C} / C} .
$$

On the other hand $s_{Z}(C)-\operatorname{Ram}_{\widetilde{C} / C} \geq 1$ if it's non-zero so if $K_{\mathcal{F}} \cdot C<0$ then $C$ is a rational curve (smooth if the singularities are canonical) with $s_{Z}(C)=0$ or 
1, giving $K_{\mathcal{F}} \cdot C=-2$ or -1 respectively. In the former case by I.3, the normal bundle $N_{\mathcal{F}}$ is flat along $C$, and the foliation is a (possibly singular) fibration in rational curves.

Tying things together, particularly in the context of II.2.1, we have:

Lemma II.3.2. (characteristic zero) Let $(X, \mathcal{F})$ be a foliated surface with $X$ non-singular and $C$ a curve on which $K_{\mathcal{F}} \cdot C<0$ then either,

(a) $C$ is not invariant, and nef.

(b) $C$ is an invariant rational curve with $K_{\mathcal{F}} \cdot C \in\{-1,-2\}$.

\section{II.4. Miyaoka's semi-positivity theorem}

We now arrive at the main application of $p$-closure, i.e. Miyaoka's semipositivity theorem, which by virtue of II.3 we'll render a little tidier, i.e.

Proposition II.4.1. [Mi2] Let $(X, \mathcal{F})$ be a foliation by curves enjoying canonical singularities on a non-singular surface in characteristic zero with $M$ a nef. $\mathbb{R}$ divisor and $C$ a curve with $K_{\mathcal{F}} \cdot C<0$ then for every $x \in C$ there is a rational curve $L_{x} \ni x$ invariant by $\mathcal{F}$ with $K_{\mathcal{F}} \cdot L_{x} \geq-2$ and,

$$
M \cdot L_{x} \leq 4 \frac{M \cdot C}{-K_{\mathcal{F}} \cdot C} .
$$

Proof. Firstly suppose $C$ is invariant, then we take $L_{x}=C$, and we're done by II.3.1(b). So we're reduced by II.3.1(a) to $C$ non-invariant and nef, which itself is just the limiting case of [S-B] 9.0.1, which one proves by combining II.1.2, II.2.1, and the usual bend \& break estimates, cf. op. cit.

Proposition II.4.1 for arbitrary algebraic spaces will involve a more careful adjunction formula which we postpone until Chapter III. Observe, however,

Corollary II.4.2. Let $(X, \mathcal{F})$ be a foliation on a 2-dimensional algebraic space with $K_{\mathcal{F}} \cdot D<0$ for some nef. $\mathbb{R}$-divisor $D$. Then for any nef. $\mathbb{R}$-divisor $M$ and $x \in X$ there is a rational invariant curve $L_{x}$ through $x$, with,

$$
\text { M. } L_{x} \leq 4 \frac{M \cdot D}{-K_{\mathcal{F}} \cdot D}
$$


where the above intersections are understood in the sense of Mumford.

Proof. Let $p:(Y, \mathcal{G}) \rightarrow(X, \mathcal{F})$ be a smooth projective resolution with canonical singularities then $p^{*} D$ and $p^{*} M$ are still nef. $\mathbb{R}$-divisors, and better still, $K_{\mathcal{G}} \cdot p^{*} D=K_{\mathcal{F}} \cdot D<0$ from which the corollary follows.

Corollary II.4.3. (Cone theorem) Let $(X, \mathcal{F})$ be a foliation on a non-singular algebraic surface then there are finitely many invariant rational curves $L_{i}$, with $K_{\mathcal{F}} \cdot L_{i} \in\{-1,-2\}$ (the latter occurring only if the foliation is a pencil of rational curves) such that,

$$
N E_{1}(X)_{\mathbb{R}}=N E_{K_{\mathcal{F}} \geq 0}(X)+\sum_{i} \mathbb{R}_{+}\left[L_{i}\right]
$$

where $N E_{1}(X)_{\mathbb{R}}$ is the cone of effective curves, and $N E_{K_{\mathcal{F}} \geq 0}$ the upper half space on which $K_{\mathcal{F}}$ is positive. Even better every $K_{\mathcal{F}}$ negative extremal ray in $N E$ is of the form $\mathbb{R}_{+}\left[L_{i}\right]$, for some $i$.

Proof. Indeed, [K1] III.1.2, this is a formal corollary of II.4.1.

\section{II.5. An alternative approach}

An equivalent characterisation of $p$-closed foliations is that they're defined by infinitesimal flat groupoids of height $p$, which is ultimately why the quotient exists and leads to the key result of this chapter II.4.1. In characteristic zero, the formal Frobenius theorem allows one to identify a foliation at it's smooth points with an infinitesimal groupoid, and after blowing up in the singularities, $\widetilde{X} \rightarrow X$ one can define a (very far from flat) infinitesimal groupoid, $F \rightrightarrows \widetilde{X}$, with source and sink $s, t$, say. Now, the non-flatness not withstanding, given a map $f: C \rightarrow X$ not factoring through the singularities from a smooth curve, one lifts it to $\tilde{f}$ to $\widetilde{X}$, and forms the fibre product,

$$
F_{C}:=F_{s} \times_{\tilde{f}} C
$$

which for any Gorenstein foliation is a smooth formal surface with trace $C$, which itself is a section of $s: F_{C} \rightarrow C$, and $N_{C / F_{C}}=f^{*} T_{\mathcal{F}}$. Critically,

Fact II.5.1. ([BM]) If $K_{\mathcal{F} \cdot f} C<0$, then the field $\mathbb{C}\left(F_{C}\right)$ of rational functions on $F_{C}$ has degree 2 . 
Proof. (cf. op. cit. II.1.1) The hypothesis imply that $N_{C / F_{C}}$ is ample, and one just counts the number of sections of any line bundle, $L$, by inductive filtering of $F_{C}$. In particular $h^{0}\left(F_{C}, L^{\otimes n}\right)$ grows at most like $n^{2}$, so the transcendence degree is at most 2 , but, obviously at least 2 by virtue of the map to $X$.

The upshot of which is,

Corollary II.5.2. To prove II.4.1 (in any dimension, and supposing only that the foliation is Gorenstein and $C$ isn't in the singular locus, which is automatic in dimension 2) we can actually suppose that our foliation is a map from an algebraic surface $S$ to $C$, and the curve in question is a section, around which the foliation is smooth.

Needless to say, this isn't terribly difficult, and admits a purely characteristic zero proof (i.e. for II.4.1 in the hypothesis of II.5.2 see [S1]), although it's still true, [S2], that characteristic $p$ is the most elegant way to go from II.5.2 to II.4.1. Better still, on replacing $X$ by it's Gorenstein covering stack, this argument works in maximal generality, i.e. $\mathbb{Q}$-Gorenstein foliations, cf. [M5] II.3. As such, the question may arise as to why this chapter has placed such emphasis on positive characteristic and $p$-closure. That historically Miyaoka's approach predated [BM] is one reason, but the deeper one is II.2.2, which is relevant to the deeper aspects of classification, cf V.5.

\section{Minimal models}

III.0. $K_{\mathcal{F}}$ vs. $K_{X}$

As observed post I.2.5, a pencil of lines through a point in $\mathbb{P}^{2}$ is a log-canonical singularity, so we have a foliated surface $(X, \mathcal{F})$ together with a modification $p:(\widetilde{X}, \widetilde{\mathcal{F}}) \rightarrow(X, \mathcal{F})$ obtained by blowing up the singularity with exceptional divisor $E$, say, such that $K_{\widetilde{\mathcal{F}}} . E=1$. Hence this singularity is $K_{X}$-terminal but we have no desire to blow such curves down if we wish to construct a minimal model, whence, unless stated otherwise, we suppose,

Hypothesis III.0.1. All foliated surfaces $(X, \mathcal{F})$ appearing in this section will be assumed to have canonical $\left(K_{\mathcal{F}}\right)$ singularities. Any precisions on the singularities of $X$ will be made according to the situation. For simplicity we will suppose that 
$(X, \mathcal{F})$ is not a fibration by rational curves.

Now we need a few definitions, viz,

Definitions III.0.2. Let $(X, \mathcal{F})$ be a foliated 2-dimensional algebraic space, $C$ a curve in the smooth locus of $X$, and $s_{\operatorname{sing}(\mathcal{F})}(C)$ as per II.3.1 then,

(a) $C$ is said to be a-1F-curve (respectively $-2 F$-curve) if $C$ is a smooth rational integral sub-curve of $\mathcal{F}$ with $s_{\operatorname{sing}(\mathcal{F})}(C)=1$ (respectively 2 ).

(b) A-1-chain is a chain of smooth rational $\mathcal{F}$ invariant curves of the form,

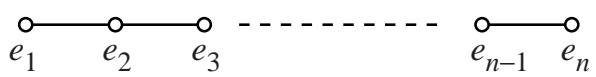

i.e. the nodes of the weighted dual graph, are starting from the handle on the far left $a-1 F$-curve, with the others $-2 F$-curves. The components will be labelled $E_{i}, i \geq 1$, starting from the handle, with $-e_{i}$ their self intersection

(c) If there exists an integral sub-curve of $\mathcal{F}$ not contained in a-1-chain but which nevertheless meets the chain then it is called the tail. By I.2.6 if it exists then such a curve is unique.

(d) A-1-chain is said to be artificial if it may be contracted to a non-singular point of the underlying space $X$. If moreover this point is actually a non-singular point of the foliation then we say that the chain is very artificial.

Notice that by II.3.1, $C$ is a $-1 F$-curve iff $K_{\mathcal{F}} \cdot C=-1$. Unsurprisingly they are the correct analogue of -1 in the usual sense. On the other hand $-2 F$-curves are a little more subtle, certainly they satisfy $K_{\mathcal{F}} \cdot C=0$, however, not only is the converse false but a $-2 F$-curve need not be contractible- consider, for example, a product of curves.

Finally we introduce a class of curves without a strict $K_{X}$ analogue, albeit that they appear in a log-variant, i.e.

Definition III.0.3. Let $(X, \mathcal{F})$ be an algebraic space, a possibly reducible but reduced curve $Z$ contained in the smooth locus of $X$ is said to be an elliptic Gorenstein leaf (e.g.l.) if it consists of a contractible cycle of -2F-curves or alternatively a contractible rational integral sub-curve with a simple node at a point of $\operatorname{sing}(\mathcal{F})$ without nilpotent structure. If it contains no curves of self- 
intersection -1 , then we say additionally that $Z$ is minimal.

The terminology is easily justified since by I.2.6 there are at most two formal invariant branches through a canonical singularity. On the other hand after a series of smooth contractions we can easily assume that our e.g.l. $Z$ is minimal, and of course has euler characteristic zero. Consequently $Z$ is the fundamental cycle of a contraction to an elliptic Gorenstein singularity. Now the unfortunate thing is (cf. IV.2.2) is that $Z$ will not be contractible to a foliated Gorenstein singularity. The following is standard,

Fact III.0.4. Let $\pi:(X, \mathcal{F}) \rightarrow(Y, \mathcal{G})$ denote the contraction in the category of algebraic spaces of a minimal e.g.l. $Z$ to an elliptic Gorenstein singularity $p$ then $Y$ is $\mathbb{Q}$-foliated Gorenstein at $p$ iff $\left.K_{\mathcal{F}}\right|_{Z}$ is torsion.

Proof. We write $Z=\sum_{i} Z_{i}$, and observe $K_{\mathcal{F}} \cdot Z_{i}=0 \forall i$. The only if part is clear, so all we need do is calculate $\pi_{*} K_{\mathcal{F}}^{\otimes n}$ for any $n \in \mathbb{N}$ with $\mathcal{O}_{Z}\left(n K_{\mathcal{F}}\right) \stackrel{\sim}{\longrightarrow} \mathcal{O}_{Z}$. On the other hand the theorem of formal functions yields,

$$
\pi_{*} K_{\mathcal{F}}^{\otimes n}=\underbrace{\lim }_{k} H^{0}\left(\mathcal{O}_{Z^{(k)}}, K_{\mathcal{F}}^{\otimes n}\right)
$$

where $Z^{(k)}$ is the $k$-th thickening of $Z$, and we have an exact sequence,

$$
H^{0}\left(\mathcal{O}_{Z^{(k+1)}}, K_{\mathcal{F}}^{\otimes n}\right) \rightarrow H^{0}\left(\mathcal{O}_{Z^{(k)}}, K_{\mathcal{F}}^{\otimes n}\right) \rightarrow H^{1}\left(\mathcal{O}_{Z}\left(n K_{\mathcal{F}}-k Z\right)\right)
$$

where the final group is isomorphic to $H^{0}\left(\mathcal{O}_{Z}(k Z)\right)=0$, and so we conclude $\pi_{*} K_{\mathcal{F}}^{\otimes n}$ has a nowhere vanishing section in a neighbourhood of $p$ as required.

\section{III.1. Algebraic spaces and local theory}

We work first of all in the category of algebraic spaces in order to compute what we must necessarily contract later in the projective category. We will freely use Mumford's intersection theory, and all identities involving "divisors" should therefore be understood modulo numerical equivalence in this sense. We proceed to the required generalisation of II.4.1, beginning with,

Lemma III.1.1. Let $p:(\widetilde{X}, \widetilde{\mathcal{F}}) \rightarrow(X, \mathcal{F})$ be a map between foliated 2-dimensional normal algebraic spaces contracting an irreducible curve $E$, and let $C$ in $X$ be 
a curve with $\widetilde{C}$ its proper transform then,

$$
\left(K_{\mathcal{F}}+C\right) \cdot C=\left(K_{\widetilde{\mathcal{F}}}+\widetilde{C}\right) \cdot \widetilde{C}+\frac{(\widetilde{C} \cdot E)}{\left(-E^{2}\right)}\left\{\left(\widetilde{C}+K_{\widetilde{\mathcal{F}}}\right) \cdot E\right\} .
$$

Proof. Apply the definition of Mumford's intersection theory. $\square$

Now let's get underway, and suppose $(X, \mathcal{F})$ is as in III.0.1, albeit with $X$ non-singular, and $E$ a curve with $K_{\mathcal{F}} . E<0$. As we have seen in II.4.1 it must be a $-1 F$-curve, and better still by II.4.1 it is contractible. We wish to contract it and see what happens. The result is best summarised by,

Proposition III.1.2. Let $(X, \mathcal{F})$ be as above and $(Y, \mathcal{G})$ a foliation on a normal 2-dimensional algebraic space obtained from $(X, \mathcal{F})$ by contracting a-1-chain with $C$ a curve in $Y$ whose proper transform $\widetilde{C}$ meets the chain and $K_{\mathcal{G}} C<0$ then $\widetilde{C}$ is a-2F-curve which is in fact the tail of the given chain and as such fits into a new-1-chain which is itself contractible.

Proof. We make use of the intermediary space obtained by contracting all but the last curve in the chain. Let's call this $(Z, \mathcal{H})$ and let $C^{\#}$ be the proper transform of $C$ in $Z$, then by III.1.1 we have on denoting by $E$ the push-forward to $Z$ of the last curve in the chain,

$$
\left(K_{\mathcal{G}}+C\right) \cdot C=\left(K_{\mathcal{H}}+C^{\#}\right) \cdot C^{\#}+\frac{\left(C^{\#} E\right)}{\left(-E^{2}\right)}\left\{\left(C^{\#}+K_{\mathcal{H}}\right) \cdot E\right\}
$$

Our aim is to show that for $C$ not invariant by $\mathcal{G},\left(K_{\mathcal{G}}+C\right) . C \geq 0$. This will easily follow by induction if we can show that the hypothesis $C^{\#} E \neq 0$ implies $\left(C^{\#}+K_{\mathcal{H}}\right) . E \geq 0$. This latter statement is itself however immediate by induction on the length of the chain. Consequently if $C$ is not an integral sub-curve of $\mathcal{G}$ then its pull-back to $X$ is a nef. $\mathbb{Q}$-Cartier divisor whose intersection with $K_{\mathcal{F}}$ is $K_{\mathcal{G}} \cdot C$ which is negative, so absurd by II.4.2.

Now given $C$ is an integrable sub-curve of $\mathcal{G}$ meeting the chain, by I.2.6 this can only happen if $C^{\#}$ meets $E$ in a singular point of $\mathcal{H}$ but not of $Z$, and on $E$ there is a distinct, possibly singular, point of $Z$ arising from contractions. Not surprisingly adjunction for $\mathcal{H}$ yields,

$$
K_{\mathcal{H}} \cdot C^{\#}=K_{\mathcal{F}} \cdot \widetilde{C}=2 g(\widetilde{C})-2+s_{\operatorname{sing}(\mathcal{H})}\left(C^{\#}\right)
$$


while on the other hand,

$$
0>K_{\mathcal{G}} \cdot C=K_{\mathcal{H}} \cdot C^{\#}+\frac{\left(K_{\mathcal{H}} \cdot E\right)}{\left(-E^{2}\right)} .
$$

Better still repeated application of the latter formula shows that if $A$, say, is the intersection matrix of the chain used to obtain $Z$, then,

$$
K_{\mathcal{H}} \cdot E=\frac{-1}{\operatorname{det}(-A)}
$$

since $\operatorname{det}(-A)$ is a positive integer we must therefore have, $K_{\mathcal{F}} \cdot \widetilde{C} \leq 0$. Given that $\widetilde{C}$ meets $\operatorname{sing}(\mathcal{F})$ this can only happen if it is a $-1 F$-curve or a $-2 F$-curve. However the former possibility contradicts I.3.2, and II.4.2.

Remarks III.1.3. The proof establishes a number of additional facts, viz: let $(X, \mathcal{F}),(Y, \mathcal{G})$ be as given with $\widetilde{C}$ the tail of the -1 -chain if it exists, $C$ its push-forward to $Y$, and $A$ the intersection matrix of the chain, then,

(a) $K_{\mathcal{G}} \cdot C=K_{\mathcal{F}} \cdot \widetilde{C}-\operatorname{det}(-A)^{-1}$.

(b) If $\widetilde{C}$ is a $-2 F$-curve then $K_{\mathcal{G}} \cdot C<0$ and we have a -1 -chain obtained by adding $\widetilde{C}$ to the original chain which is itself contractible.

(c) Any two -1-chains of maximal length are disjoint. Otherwise we'd have the situation eliminated in the proof, i.e. a tail which is a $-1 F$ curve.

(d) A -1-chain is contractible.

Finally let us note a fact which will ultimately prove rather useful, viz:

Proposition III.1.4. A contraction of a-1-chain containing no exceptional curves of the $1^{\text {st }}$ kind inside a foliated surface $(X, \mathcal{F})$ with underlying space $X$ non-singular cannot be foliated Gorenstein.

Proof. Let $\pi:(X, \mathcal{F}) \rightarrow(Y, \mathcal{G})$ be the contraction of the chain supported on $E_{1}, \ldots, E_{n}$ say, and suppose $K_{\mathcal{G}}$ is foliated Gorenstein. A priori we know there are $a_{i} \in \mathbb{Q}_{>0}$ such that,

$$
K_{\mathcal{F}}=\pi^{*} K_{\mathcal{G}}+\sum_{i} a_{i} E_{i}
$$


as $\mathbb{Q}$-Cartier divisors. Now if $K_{\mathcal{G}}$ is Cartier then, $a_{1}=K_{\mathcal{F}} \cdot \pi^{*} K_{\mathcal{G}}-K_{\mathcal{F}}^{2} \in \mathbb{Z}$ furthermore, $-1=a_{1} E_{1}^{2}+a_{2}$ so in addition $a_{2} \in \mathbb{Z}$, and one sees easily by induction that indeed all the $a_{i} \in \mathbb{Z}$. Conversely, if $d$ is the discriminant of the negative of the chain's intersection matrix then, $a_{n}=1 / d$, which is absurd.

\section{III.2. Contractions}

We begin with $(X, \mathcal{F})$ as in III.0.1 with underlying space $X$ non-singular. If $K_{\mathcal{F}}$ is nef. we do nothing. Otherwise there is a -1 -chain, and by a usual Néron-Severi type finiteness argument, any -1-chain is contained in one of maximal length, and as observed these are all disjoint. Should there be a -1-chain which is artificial then we contract it, and so inductively obtain a foliated surface $\left(X^{\prime}, \mathcal{F}^{\prime}\right)$ obtained by contracting on $(X, \mathcal{F})$ with $X^{\prime}$ non-singular which contains no artificial -1-chains. Thus if $K_{\mathcal{F}^{\prime}}$ is not nef. there is a finite disjoint union of maximal -1-chains none of which is artificial. A priori these can be contracted simultaneously to normal singularities on an algebraic space, however since they are chains of rational curves it is standard, cf. $[\mathrm{A}]$, that the unique space $(Y, \mathcal{G})$ obtained by this contraction is a $\mathbb{Q}$-factorial projective variety with rational singularities, so all previous calculations in Mumford intersection theory remain valid in the Picard group with $\mathbb{Q}$-coefficients. Evidently $(Y, \mathcal{G})$ has canonical singularities, and so we require to prove,

Proposition III.2.1. With $(Y, \mathcal{G})$ as above, $K_{\mathcal{G}}$ is nef.

Proof. Without loss of generality, we may, in the above notations, replace $(X, \mathcal{F})$ by $\left(X^{\prime}, \mathcal{F}^{\prime}\right)$. We let $C$ be a curve in $Y$ with $K_{\mathcal{G}} \cdot C<0$ and $\widetilde{C}$ its proper transform in $X$. A minor variation of the argument in III.1.2 reduces us to the case that $C$ is an integral sub-curve of $\mathcal{G}$. Moreover if $C$ is not the tail of any of the -1-chains that we have contracted then $(X, \mathcal{F})$ is isomorphic to $(Y, \mathcal{G})$ in a neighbourhood of $C$ and so $K_{\mathcal{G}} \cdot C=K_{\mathcal{F}} \cdot \widetilde{C} \geq 0$ which is absurd. Whence let $T_{1}, \ldots, T_{k}$ be the maximal -1-chains which have $\widetilde{C}$ as their tail and $d_{1}, \ldots, d_{k} \in \mathbb{N}$ the determinants of the negative of the intersection matrices of the appropriate chains, then we have:

$$
K_{\mathcal{G}} \cdot C=K_{\mathcal{F}} \cdot \widetilde{C}-\sum_{i=1}^{k} \frac{1}{d_{i}} .
$$

Better still none of the chains are artificial, and so, $K_{\mathcal{G}} \cdot C \geq K_{\mathcal{F}} \cdot \widetilde{C}-k / 2$. On 
the other hand we have by adjunction,

$$
K_{\mathcal{F}} \cdot \widetilde{C}=(2 g(\widetilde{C})-2)+s_{\operatorname{sing}(\mathcal{F})}(\widetilde{C}) \geq 2 g(\widetilde{C})-2+k .
$$

Consequently $C$ is a rational curve, and $k \leq 3$. Should $k=1$, then by III.1.2, $\widetilde{C}$ is a $-2 F$-curve which fits into a new -1 -chain, whence the absurdity that our given chain isn't maximal. Should $k=2$, then $\widetilde{C}$ is still a $-2 F$-curve, and so we are reduced to considering the case $k=3$. In this case $s_{\operatorname{sing}(\mathcal{F})}(\widetilde{C})=3$. Again maximal -1-chains are disjoint, while the singularities $Z_{j}$, of the foliation, give local Segre classes of 1 for both $\widetilde{C}$ and the chain, so they cannot carry any nilpotent structure, whence by I.3.2, $C^{2} \geq 0$ contrary to II.4.2.

Remark III.2.2. If we extend the discussion to an invariant $C$ whose proper transform meets several maximal non-artificial chains and $K_{\mathcal{G}} \cdot C=0$, then, in the above notation, $k \leq 4$. The case $k=1$ is impossible, while $k=3$ or 4 imply by an identical argument to the above that $C^{2} \geq 0$.

As hinted $k=2$ merits further discussion. In the above notations this can only happen if $d_{1}=d_{2}=2$. Consequently by systematically contracting rational curves of self-intersection -1 appearing in either chain, we may replace $(X, \mathcal{F})$ by a smaller model in which the underlying space is still non-singular and both chains in question consist of a single curve with self-intersection -2 . In principle it might occur that the intersection of $\widetilde{C}$ with the singular locus of $\mathcal{F}$, although 3 , due to nilpotent structure on $\operatorname{sing}(\mathcal{F})$ is supported at only 2 points, but this is excluded by I.3.1. The remaining case is more delicate, i.e. the singular locus of $\mathcal{F}$ meets $\widetilde{C}$ in 3 -distinct points, and so there could be an integrable sub-curve meeting $\widetilde{C}$ in the point not determined by the -1 -chains. Note, though that $C^{2}=\widetilde{C}^{2}+1$, is an integer. Whence we make,

Definition III.2.3. (b) A curve $\widetilde{C}$ of the above form is said to be a bad tail.

\section{III.2.bis. The stack alternative}

The whole of the previous discussion is radically clearer if one works systematically in the 2-category of algebraic stacks, and indeed the particular features of small dimension in the previous discussion would allow us to confine our attention to orbifolds. Specifically, as we've said, it follows from a variant of II.5.1/2 that II.4.1/2 is true on the Gorenstein covering stack $(\mathcal{X}, \mathcal{F})$ of any $\mathbb{Q}$-Gorenstein 
foliated normal surface, $(X, \mathcal{F})$. Apart from this, we also need the adjunction formula II.3.1, which as stated is valid on any Gorenstein variety, and is equally valid on any Gorenstein stack in the form,

Precision III.2.bis.1. Let $f: \mathcal{C} \rightarrow \mathcal{X}$ be a map from a smooth 1-dimensional stack to a Gorenstein foliated stack not factoring through the singular locus of the foliation $\mathcal{F}$, and invariant by it, then,

$$
K_{\mathcal{F} \cdot f} \mathcal{C}=-\chi(\mathcal{C})+s_{Z}(\mathcal{C})
$$

where the former is the Euler characteristic of $\mathcal{C}$ (equally the orbifold Euler characteristic if $\mathcal{C}$ is generically scheme like), and the latter is the Segre class in the stack sense, i.e. adjusted for non-scheme like points, cf. [M5] I.8.6.

As such, III.1.3 (a), and to a lesser extent III.1.1, are in fact III.2.bis.1. Consequently, an alternative schema of proof is,

Stack version III.2.bis.2 (cf. [M5] IV) Let $(\mathcal{X}, \mathcal{F})$ be a smooth foliated algebraic stack of dimension 2 with canonical singularities and non-scheme like locus transverse to $\mathcal{F}$, then if the foliation isn't a pencil of rational (i.e. positive euler characteristic) stacks we may construct a minimal model by,

(a)If $K_{\mathcal{F}}$ is not nef. then as per II.4.3 there is an invariant rational stack $\mathcal{L}$ with $K_{\mathcal{F}} \cdot \mathcal{L}<0$. By II.4.2 $\mathcal{L}$ is contractible, and by I.2.6 smooth.

(b)Contracting $\mathcal{L}$ yields a terminal singularity, so by I.2.1 at worst a quotient singularity of the ambient space, i.e., the moduli of the contraction admits a unique smooth stack structure $\mathcal{X}_{1}$ isomorphic to our initial one outside of $\mathcal{L}$, while preserving the initial structural hypothesis for the induced foliation $\mathcal{F}_{1}$.

(c) Repeat stage (a), and observe that at each stage Néron-Severi goes down by one, so as to obtain a minimal model $(\mathcal{Y}, \mathcal{G})$. Further all the 'new' singularities are terminal, so, a postiori, by II.2.2, the minimal smooth resolution of $\mathcal{Y}$ 's moduli differs from that of $\mathcal{X}$ precisely by finitely many disjoint -1 chains.

This in turn leads to,

Reinterpretation III.2.bis.3. The observations III.1.3 admit, in order, the following re-interpretation on the Gorenstein covering stack $(\mathcal{Y}, \mathcal{G}) \rightarrow(Y, \mathcal{G})$,

(a) The pull-back $\widetilde{\mathcal{C}}$ of $\widetilde{C}$ to $\mathcal{Y}$ has one non-scheme like point (where by the way 
the induced foliation is smooth) with stabiliser of order $\operatorname{det}(-A)$. By I.2.2, the stabiliser is in fact cyclic.

(b) An invariant curve $\widetilde{\mathcal{C}}$ in $\mathcal{Y}$ with $K_{\mathcal{G}} \cdot \widetilde{\mathcal{C}}<0$ is contractible.

(c) An invariant rational stack $\widetilde{\mathcal{C}}$ in $\mathcal{Y}$ must meet $\operatorname{sing}(\mathcal{G})$. Indeed, otherwise just like a smooth invariant rational curve inside the smooth locus of a foliation on a smooth variety it (more correctly it's universal covering) would move, or alternatively $\widetilde{\mathcal{C}^{2}}=0$ by I.3.2, and apply II.4.2.

The stack approach is therefore numerically much less fastidious, and,

Remarks III.2.bis.4 Furthermore algebraic stacks allow us,

(a) To treat the case of geometric boundaries (pre I.1.10) exactly as one treats empty boundaries, with the (non algebraic stack) case of weight 1 boundaries, following from the weight $\frac{1}{2}$ case, cf. [M5] IV.9.

(b) They present a better explanation for the termination of contraction than the decrease of the rank of Néron Severi. Specifically, by II.2.1 the number of singular points of the foliation decrease under terminal contractions, and this is why flipping in higher dimension terminates, cf. [M5] IV.7.

(c) Needless to say the schema of proof III.2.bis.1 works for any smooth 2 dimensional algebraic stack, i.e. with no prescriptions on the location of the non-scheme like points. It also works for log-canonical singularities, not just canonical since this hypothesis is only used to guarantee the smoothness of the rational stack that is being contracted. If, however, the rational stack were not smooth then by I.2.4 we could resolve the log-canonical singularity by a single weighted blow up, apply the stack version of I.3.2 on the resolution, and deduce from II.4.2 that our foliation is a pencil of rational curves. Again this is true in all dimensions, cf. [M5] IV.8.

\section{III.3. Proof of the canonical model theorem}

Keeping to the notations of the previous section we start with a foliated surface $(X, \mathcal{F})$ containing no artificial -1-chains, and contract all non-artificial -1-chains to obtain a foliated surface $(Y, \mathcal{G})$ with canonical singularities. By III.1.3 no $-2 F$-curve can meet a non-artificial chain. Better still the set of 
$-2 F$-curves is not only finite, but the union of its members is a disjoint union whether of chains, or cycles and indeed there may be rational integral sub-curves with a node. Regardless concentrating on the first fact we may freely contract $-2 F$-curves with self-intersection -1 . This step may be carried out in either $X$ or $Y$ without changing $K_{\mathcal{F}}$ or $K_{\mathcal{G}}$. The former is perhaps preferable, so let us add to our hypothesis that in $X$ there are no $-2 F$-curves with self-intersection -1 . We extend the weighted dual graph notation of III.0.2 to chains or cycles of $-2 F$-curves in the obvious way. For such graphs, or indeed any connected subgraph, we may form the associated quadratic form, which we call contractible if it is positive definite. Since the possibility of a node of weight one is excluded if $C$ is a curve corresponding to a positive node then it is contained in a unique maximal contractible sub-chain or cycle.

With this in mind we now contract once more all -1-chains in $(X, \mathcal{F})$ to get $(Y, \mathcal{G})$ with $K_{\mathcal{G}}$ nef. As ever the appropriate variation of III.1.1 guarantees what will be the defining property of the canonical model, viz,

Definition III.3.1 A foliated normal surface $(Y, \mathcal{G})$ with canonical singularities is said to be a canonical model if $K_{\mathcal{G}}$ is nef, and, $K_{\mathcal{G}} \cdot C=0 \Rightarrow C^{2} \geq 0$.

while if $C$ is not a tail yet an integral sub-curve the property holds unless $C$ is a $-2 F$-curve or an e.g.l. supported on a rational curve with a node. We wish to contract these but the possibility of bad tails necessitates two cases.

Let us therefore consider bad tails. Even if they exist they're either contractible or they're not, and we conclude to III.3.1 unless either a bad tail intersects a contractible chain of $-2 F$-curves, or indeed that bad tails intersect among themselves. Extending the previous conventions on dual graphs to this situation there are therefore three possibilities in $X$, 
a)

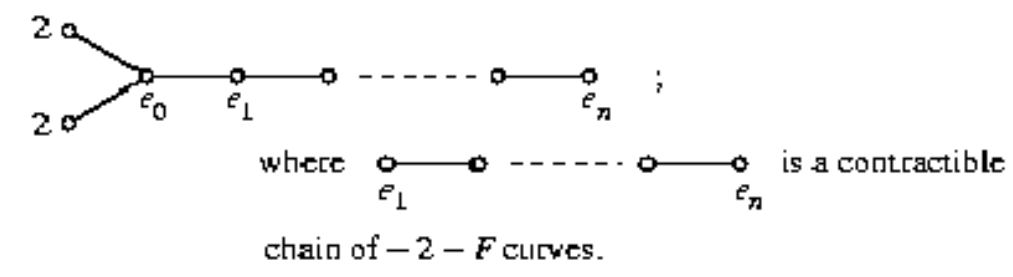

b)

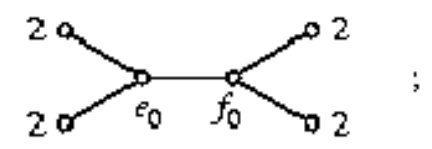

where the nodes corresponding to $e_{0}$ and $f_{0}$ are bad tails.

c)

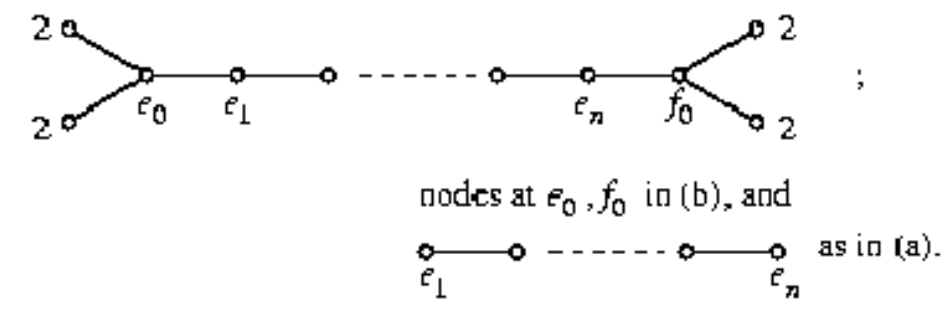

Now case (a) is simply either contractible or it is not, according to whether $e_{0} \geq 2$ or not. In any case if it is contractible then it is contractible to a rational $\mathbb{Q}$ factorial singularity as in $[\mathrm{A}]$. Evidently if it's not completely contractible then contracting the $-1 F$ curves, and the $e_{1}, \ldots, e_{n}$ chain gives the defining property. Turning to cases (b) and (c) we know these cannot be contractible by I.3.2. Now if both $e_{0}, f_{0} \leq 1$ then we just contract the $-1 F$ curves, and the $e_{1}, \ldots, e_{n}$ chain to get the defining property. Otherwise say $e_{0} \geq 2$, and we contract the subgraph of the form (a) and the remaining $-1 F$ curves to obtain the conclusion.

We have thus shown that a foliated surface $(X, \mathcal{F})$ with underlying space non-singular admits a map to a canonical model with the given defining characteristics, and rather more generally a minor variant of the same argument establishes,

Theorem 1. III.3.2 (Canonical model theorem.) Let $(X, \mathcal{F})$ be a 2-dimensional foliated normal algebraic space with canonical foliation singularities then there is a proper birational map $\pi:(X, \mathcal{F}) \rightarrow(Y, \mathcal{G})$ to a canonical model with 
canonical foliation singularities satisfying III.3.1 The underlying space $Y$ has at worst rational quotient singularities or elliptic Gorenstein singularities, as such there is a unique minimal desingularisation $\rho:\left(Y^{\prime}, \mathcal{G}^{\prime}\right) \rightarrow(Y, \mathcal{G})$ whose structure over a singular fibre is one of the following mutually exclusive types,

(i) A chain of rational integrable sub-curves represented by its dual graph,

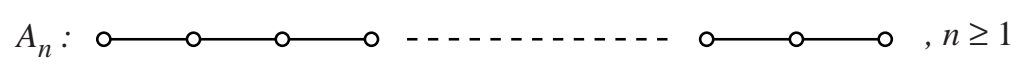

consisting either of a-1-chain (III.0.2), two $-1 F$-curves of square -2 joined by a bad tail (III.2.3) or a chain of -2 F-curves (III.0.2).

(ii) Two - $1 F$-curves of square -2 joined by a bad tail which itself connects to a chain of -2 F-curves (III.2.3) and forms the dual graph,

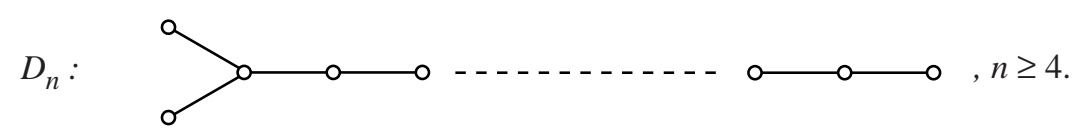

(iii) A basic elliptic Gorenstein leaf which is not irreducible, i.e. a cycle of $-2 F$-curves forming the fundamental cycle of an elliptic Gorenstein singularity (III.0.3), i.e. the dual graph,

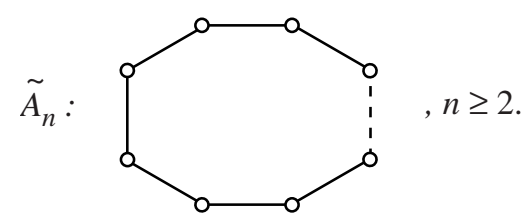

(iv) The irreducible elliptic Gorenstein leaf, i.e. a rational integral sub-curve with a node which scheme theoretically has Segre class (cf. II.3) precisely two at the singular locus of $\mathcal{G}^{\prime}$.

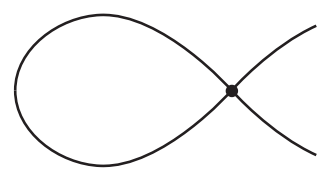

Furthermore the structure of $K_{\mathcal{G}^{\prime}}$ is of the form, $K_{\mathcal{G}^{\prime}}=\pi^{*} K_{\mathcal{G}}+\sum E_{i}$ where each $E_{i}$ is a divisor whose support is a maximal-1-chain as indicated in (i) above. In particular there is a $\mathbb{Q}$-factorial model on which $K_{\mathcal{G}}$ is nef.

We subordinate/postpone a detailed discussion of uniqueness to classification, and for the moment content ourselves with, 
Complement III.3.3. If $(Y, \mathcal{G}),\left(Y^{\prime}, \mathcal{G}^{\prime}\right)$ are canonical models of foliated surfaces and $p:\left(Y^{\prime}, \mathcal{G}^{\prime}\right) \rightarrow(Y, \mathcal{G})$ a map between them which is defined everywhere then $p$ is an isomorphism.

Proof. Since $Y, Y^{\prime}$ are normal, it suffices to check that it is injective. Should it fail to be injective then some point $y \in Y$ has a one-dimensional connected fibre, $F$, say. However $(Y, \mathcal{G})$ is canonical, and $K_{\mathcal{G}^{\prime}}$ is nef. so $K_{\mathcal{G}^{\prime}} \cdot E=0$ for all components $E$ of $F$, which by the defining property contradicts the negative definite nature of the intersection form on $F$.

\section{III.3.bis. The Stack approach}

Again, we can profit from the fact that the Gorenstein cover of a minimal model is a smooth stack, and indeed on any smooth 2 dimensional foliated stack, $(\mathcal{X}, \mathcal{F})$ with $\mathcal{C}$ a non-invariant irreducible curve, one trivially has,

$$
\left(K_{\mathcal{F}}+\mathcal{C}\right) \cdot \mathcal{C} \geq 0
$$

Consequently, any curve that we need to contract to guarantee the canonical model condition III.3.1 is necessarily uniformised by a rational stack by III.2.bis.1. Such a curve can only meet the singular locus of $\mathcal{F}$ in at most two points, so by I.2.6 the image is either smooth or has a node. Such a curve is either contractible, or it's not. If the image is smooth, then after contraction the moduli continues to admit a smooth stack structure with canonical singularities. Consequently, the only novelty is the contraction of rational curves with a node. That the contraction of such curves is a final step, is clear by I.2.6, and the augmentation of the square of any curve under any contraction. This establishes the existence of a canonical model for any algebraic stack, which is everywhere smooth outside the contraction of finitely many egl's.

A postiori, one can then compare II.2.2 and II.2.4 with III.3.2. Evidently the contraction of a $-1-F$ chain is just the terminal singularity II.2.2. Two $-1-F$ curves joined by a bad tail correspond to II.2.4 (e) with an abelian group action, and the contraction of a chain of $-2-F$ curves is any of II.2.4 (a)-(d). As such the case of $D_{n}, n \geq 4$, is the resolution of II.2.4 (e) for a non-abelian group action, while III.3.2 (iii) and (iv) are essentially the same from the stack point of view, they are nevertheless new since as we'll see in IV.2.2, they are never Gorenstein. 
In particular, III.3.2 is also an exhaustive list of the minimal resolutions of canonical foliation singularities on surfaces. Regrettably, a log-canonical analogue looks rather more involved as I.2.5 indicates since one may have to contract rational curves with a very large cusp, at which point one is arbitrarily far from a good technical restriction on the ambient space or stack.

\section{Classification and abundance}

\section{IV.0. Definitions}

The existence of minimal models leads to our basic objects of study,

Definition IV.0.1. Let $(X, \mathcal{F})$ be a model of a foliated surface with canonical singularities then we define the numerical Kodaira dimension $\nu(X, \mathcal{F})$ to be,

(i) $-\infty$ if $K_{\mathcal{F}}$ is not pseudo-effective, i.e. $\mathcal{F}$ is a fibration by rational curves.

(ii) Otherwise let $\pi:(X, \mathcal{F}) \rightarrow(Y, \mathcal{G})$ be a map to a minimal model then modulo numerical equivalence with $\mathbb{Q}$-coefficients we have,

$$
\nu(X, \mathcal{F})=\max \left\{i: K_{\mathcal{G}}^{i} \neq 0\right\}
$$

Despite the possibility of non-uniqueness it is an easy exercise in the use of the index theorem to prove this is well-defined, and whence a birational invariant. Similarly we have,

Definition IV.0.2. Let $(X, \mathcal{F})$ be as above then we define the Kodaira dimension $\kappa(X, \mathcal{F})$ to be the Kodaira dimension of $K_{\mathcal{F}}$. By standard properties of Kodaira dimension this is independent of the choice of model provided that it has canonical singularities, and as such is a birational invariant.

Finally for $(X, \mathcal{F})$ as above we put these both together and say,

Definition IV.0.3. Abundance holds for $(X, \mathcal{F})$ if $\nu(X, \mathcal{F})=\kappa(X, F) \geq 0$.

Before proceeding further, let us for convenience issue,

Warning IV.0.4. From now on we will confine our attention to foliated surfaces defined over an algebraically closed field of characteristic 0 , so, $\mathbb{C}$. 


\section{IV.1. Rationality}

Let us understand surface to be a two dimensional algebraic space, and record,

Theorem IV.1.1. Let $(X, \mathcal{F})$ be a foliated surface with canonical singularities then $(X, \mathcal{F})$ is a fibration by rational curves iff there is a curve $C$ with $K_{\mathcal{F}} \cdot C<$ -1 . The if direction holds for arbitrary foliation ingularities, but $X$ non-singular.

The necessity of the condition is obvious if $X$ is non-singular, and whence for arbitrary canonical singularities by an immediate application of the definition. The sufficiency is an application of the cases considered in III which imply it could not be otherwise. Clearly the numerical Kodaira dimension and Kodaira dimension coincide.

Intuitively one expects that $\mathbb{P}^{1}$-bundles over smooth curves (with their natural fibration) ought to be the canonical models in this situation with uniqueness up to flipping fibres. We observe this is indeed so, viz,

Complement IV.1.2. Let $(X, \mathcal{F})$ be as per IV.1.1, with $\mathcal{F}$ a fibration by rational curves, and $\pi:(Y, \mathcal{G}) \rightarrow(X, \mathcal{F})$ a resolution with canonical singularities, but $Y$ smooth, then there is a proper birational map $p:(Y, \mathcal{G}) \rightarrow\left(X^{\prime}, \mathcal{F}^{\prime}\right)$ where the latter is the natural fibration on a $\mathbb{P}^{1}$-bundle over a smooth curve.

Proof. Let $\Gamma$ be the connected curve over one of the finitely many possible singular points of $X$. After systematically contracting any artificial -1-chains in $\Gamma$, we observe that the arguments of III.1.2 and III.2.1, thanks to the contractibility of $\Gamma$, go through verbatim to show that contracting all the other -1-chains in $\Gamma$ yields a map $q:(X, \mathcal{F}) \rightarrow(Z, \mathcal{H})$ such that $q(\Gamma)$ is contractible and $K_{\mathcal{H}}$ is nonnegative on all integral sub-curves of $q(\Gamma)$ - note $\Gamma$ cannot contain leaves since these are not contractible - so as ever by III.1.1, $\Gamma$ consists of integral sub-curves. The quotient space $B=X / \mathcal{F}$, however, exists and is normal, whence a smooth projective curve. Consequently $\Gamma$ is contained in a fibre of $\rho: X \rightarrow B$. Necessarily this fibre is singular. Let $F_{1}, \ldots, F_{r}$ be the singular fibres with $\sum_{i} F_{i}=\rho^{*} D$, say, then,

$$
K_{\mathcal{F}}=K_{X}-\rho^{*} \omega_{B}(D)+\sum_{i=1}^{r}\left(F_{i}\right)_{\mathrm{red}}
$$

whence if $C$ is a general fibre, then $K_{\mathcal{F}} \cdot C=K_{X} \cdot C=-2$. Thus if $F$ is any 
singular fibre then by adjunction (for $K_{X}$ ) it contains a rational integral subcurve with $C^{2}=-1$, so by a sequence of contractions of $-1 F$ - or $-2 F$-curves we arrive at $\left(X^{\prime}, \mathcal{F}^{\prime}\right)$ provided we always give preference in contracting curves with $C^{2}=-1$ to those that may appear in the push-forward of $\Gamma$.

The attentive reader will have noticed that $K_{X}$ intervenes in this proof in an essential way to guarantee the smoothness of $X^{\prime}$. As it happens this is the only such intervention of $K_{X}$, but it's interesting to note why it happened, i.e. given a foliation which is a rational pencil with canonical singularities one can aim to follow the minimal model algorithm in the sense of contracting $-1 F$ curves, and more generally $-1 F$ chains. Such a procedure will certainly lead to a model $(Y, \mathcal{G})$ of $(X, \mathcal{F})$ whose Gorenstein covering stack, $\mathcal{Y}$, is smooth, but $Y$ itself may not be smooth. Certainly then, there is a map $\mathcal{Y} \rightarrow[\mathcal{Y} / \mathcal{G}]$, which exhibits $\mathcal{Y}$ as a $\mathbb{P}^{1}$-bundle in the étale topus of a 1 -dimensional smooth stack $[\mathcal{Y} / \mathcal{G}]$, but this is obviously not as strong as IV.1.2. Plainly this results from the highly non-unique nature of 'contracting $-1 F$ chains' in the rational case. Curiously, however, there is no such problem if there is a singularity which is log-canonical rather than canonical, i.e.

Curiosity IV.1.3. Let $(X, \mathcal{F})$ be a foliated smooth surface with log-canonical singularities not all of which are canonical and such that the operation of contracting $-1 F$-chains does not lead to a minimal model then the contraction of the said chains gives a proper map $p:(X, \mathcal{F}) \rightarrow(\mathbb{P}(p, q, 1), \mathcal{R})$, where the latter is the radial foliation on a weighted projective space, $(p, q)=1$.

As one might imagine, the log-canonical singularity in question is $p x \frac{\partial}{\partial x}+$ $q y \frac{\partial}{\partial y}$ for weighted coordinates $X, Y, Z$ of weights $p, q, 1$, i.e. a generalisation of lines through a point in the plane. Again, this is much clearer from the point of view of the Gorenstein covering stack $\mathcal{P} \rightarrow \mathbb{P}(p, q, 1)$, which has two nonscheme like points where the hyperplane at infinity meets the $x$, and $y$-axis with stabilisers $\mathbb{Z} / p$ and $\mathbb{Z} / q$ respectively. Consequently the two axis lift to so called 'bad' orbifolds with signatures $p$, and $q$ at infinity respectively, while every other invariant curve has as normalisation an honest $\mathbb{P}^{1}$. The use of the word 'bad' here is extremely misleading, since what it refers to is simply connected rational stacks. Necessarily these have moduli $\mathbb{P}^{1}$ and non-scheme like points with stabilisers $\mathbb{Z} / m$ and $\mathbb{Z} / n, m$ and $n$ relatively prime integers, where we slightly abusively allow one, 
but not both of $m$ or $n$ to be infinite (as naturally occurs when we look at weight 1 boundaries). We denote such stacks by $\mathbb{P}^{1}(m . n)$, and note,

Stacky rationality IV.1.4. (cf. [M5] I.9) Let $(\mathcal{X}, \mathcal{F})$ be a smooth foliated algebraic stack of, say, dimension 2 , to keep us in context, with log-canonical singularities then if the procedure III.2.bis.2 does not yield a model with Gorenstein covering stack $(\mathcal{Y}, \mathcal{G})$ on which $K_{\mathcal{G}}$ is nef., then either,

(a) The singularities are canonical, and $\mathcal{Y} \rightarrow[\mathcal{Y} / \mathcal{G}]$ exhibits $\mathcal{Y}$ as a fibration in algebraic stacks which is a $\mathbb{P}^{1}(m, n)$ bundle in the étale topus of $[\mathcal{Y} / \mathcal{G}]$.

(b) Not all the singularities are canonical, and $(\mathcal{Y}, \mathcal{G})$ is a radial foliation on a generalised weighted projective stack.

Here we have refrained from defining generalised weighted projective stacks, but as the words suggest, this is just some stack structure whose moduli is a weighted projective space, where we continue to allow the possibility of one of the weights being infinite (cf. op. cit. I.9 and IV.8). A picture, however, is better than a thousand words, and in this respect one might consider an example very close to IV.1.3. Indeed if we introduce some geometric weight $1-\frac{1}{a}$ at infinity, and replace our original $p, q$ by $a_{1}=p a, a_{2}=q a$, then the (generalised) contraction of $-1 F$ -chains leads to the weighted projective stack with stabilisers $\mathbb{Z} / a$ at the generic point at infinity, and $\mathbb{Z} / a_{i}$ where the axis meet infinity, so that our foliation is,

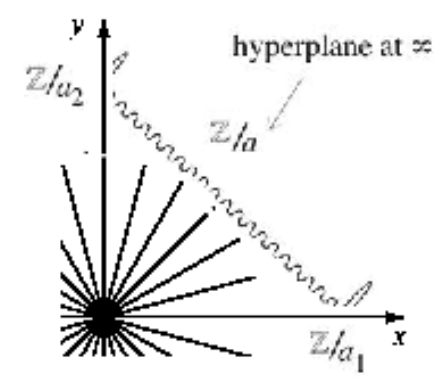

Independently of any stackiness questions we can combine IV.1.2 with a celebrated theorem of Bogomolov, [B1], to obtain an amusing corollary (already noted by M. Brunella, [Br1], in his classification of smooth foliations), 
Corollary IV.1.5. Let $(X, \mathcal{F})$ be a smooth foliated surface (i.e. the underlying space $X$ is non-singular and $\mathcal{F}$ has terminal singularities) then $X$ is minimal unless $\mathcal{F}$ is the foliation obtained from blowing up the point of common intersection of a pencil of lines in $\mathbb{P}^{2}$.

Proof. If the numerical Kodaira dimension of $(X, \mathcal{F})$ is $-\infty$, then this immediately follows by IV.1.2. Otherwise let,

$$
0 \rightarrow N \rightarrow \Omega_{X}^{1} \rightarrow K_{\mathcal{F}} \rightarrow 0
$$

define the foliation, then $(X, \mathcal{F})$ is a minimal model, so in particular $K_{\mathcal{F}}$ is nef. Now suppose $X$ is not a minimal surface and let $E$ be an exceptional curve of the $1^{\text {st }}$ kind then $N$. $E \leq-1$, whence for any positive rational number $\varepsilon$ we have,

$$
(N-\varepsilon E)^{2} \geq 2 \varepsilon-\varepsilon^{2}, K_{\mathcal{F}}(N-\varepsilon E)=c_{2}(X)-\varepsilon K_{\mathcal{F}} \cdot E .
$$

Now suppose further that $X$ is not birational to a $\mathbb{P}^{1}$-bundle over a curve of genus $g$, then since $X$ is not minimal $c_{2}(X)>0$ so that the above plus Riemann-Roch imply that for $\varepsilon$-sufficiently small $N-\varepsilon E$, and whence $N$ is big, contrary to a famous estimate of Bogomolov, cf. [B1]. Whence $X$ is a surface of the said type on which there are exceptional curves of the $1^{\text {st }}$ kind so, $c_{1}(X)^{2}<2 c_{2}(X)$. However we have the basic estimate, $c_{1}\left(X^{2}\right)-2 c_{2}(X)=K_{\mathcal{F}}^{2} \geq 0$ which is absurd.

IV.2. General type $(\nu(X, \mathcal{F})=2)$

Certainly abundance and uniqueness holds under the further hypothesis of general type but the canonical model theorem gives more, viz:

Theorem IV.2.1. Let $(X, \mathcal{F})$ be the unique canonical model of a foliation of general type and let $\pi:(Y, \mathcal{G}) \rightarrow(X, \mathcal{F})$ be the resolution of the cusps then there is an ample $\mathbb{Q}$-divisor $H$ and an effective $\mathbb{Q}$-divisor $Z$ (supported on the minimal) elliptic Gorenstein leaves such that,

$$
K_{\mathcal{G}}=\pi^{*} K_{\mathcal{F}}=H+Z .
$$

Proof. This is just the index theorem, combined with Kleiman's criterion.

Of course the presence of e.g.l.s in the above decomposition appears a priori rather ugly. Certainly one would have hoped for a base point freeness result for 
$K_{\mathcal{F}}$ on the canonical model. However eventual freeness of $K_{\mathcal{F}}$ be it on $X$ or on $Y$, in the above notations, would imply that $K_{\mathcal{F}}$ is $\mathbb{Q}$-foliated Gorenstein on the canonical model, and this in general is impossible. Indeed,

Theorem IV.2.2. Let $\pi:(Y, \mathcal{G}) \rightarrow(X, \mathcal{F})$ be the contraction of a minimal elliptic Gorenstein leaf on a foliated algebraic space with canonical singularities then the contraction is never $\mathbb{Q}$-foliated Gorenstein. Better still the germ of any such contraction is isomorphic to the germ of the natural foliation on the BailyBorel compactification of a Hilbert modular surface.

Proof. The question is completely local, so without loss of generality we may take $Y$ to be a tubular neighbourhood of the e.g.l., $Z$, say. We denote by $Z_{1}, \ldots, Z_{n}$ the components of $Z$ and proceed to calculate what it means for the contraction to be $\mathbb{Q}$-foliated Gorenstein. Fix a component $Z_{i}$, and cover the smooth locus of $\mathcal{F}$ in a neighbourhood of $Z_{i}$ by polydiscs $\Delta_{\alpha}$. Then the bundle $N$, say, in $\Omega_{Y}^{1}$ defining the foliation admits a description of the form, $\left.N\right|_{\Delta_{\alpha}}=\mathcal{O}_{\Delta_{\alpha}} d z_{\alpha}$, for $z_{\alpha} \in \Gamma\left(\Delta_{\alpha}\right)$ which may in turn be taken as defining $\mathcal{O}_{\Delta_{\alpha}}(Z)$ locally. Consider the respective transition functions $g_{\alpha \beta}, h_{\alpha \beta}$ and observe the formulae,

$$
d z_{\alpha}=g_{\alpha \beta} d z_{\beta}, z_{\alpha}=h_{\alpha \beta} z_{\beta} \Rightarrow d z_{\alpha}=h_{\alpha \beta} d z_{\beta} \quad \text { in } \quad \Omega_{\Delta_{\alpha}}^{1} \otimes \mathcal{O}_{Z} .
$$

Consequently in $\mathcal{O}_{Z} \otimes \mathcal{O}_{\Delta_{\alpha}}$ we have $g_{\alpha \beta} h_{\alpha \beta}=1$, and this represents the class in $\operatorname{Pic}(Z)$ of $\mathcal{O}_{Z}(N+Z)$. Now the intersection of $Z$ with the smooth locus of $\mathcal{G}$ is just $Z$ minus its singularities. So let $p$ be such a singularity, with $x_{p}, y_{p}$ local equations for the components (in the strict Henselisation) of $Z$ through $p$, and $\omega_{p}$ a generator of $N$ at $p$, then for a suitable polydisc $\Delta_{p}$ about $p$,

$$
d z_{\alpha}=g_{\alpha p} \omega_{p}, z_{\alpha}=h_{\alpha p}\left(x_{p} y_{p}\right) \Rightarrow d z_{\alpha}=h_{\alpha p} d\left(x_{p} y_{p}\right) \quad \text { in } \quad \Omega_{\Delta_{p}}^{1} \otimes \mathcal{O}_{Z} .
$$

Further, we note that $\omega_{p}$ is of the form, $x_{p} a_{p} d y_{p}+y_{p} b_{p} d x_{p}$, where $a_{p}(p) b_{p}(p)$ $\neq 0$, so without loss of generality $a_{p}, b_{p}$ are invertible over $\Delta_{p}$. Now by virtue of the fact that the contraction is elliptic Gorenstein $K_{Y}+Z$ may be assumed trivial. Whence $(X, \mathcal{F})$ is $\mathbb{Q}$-foliated Gorenstein by III.0.4 if and only if, $N+Z$ is torsion in $\operatorname{Pic}^{0}(Z)$. So let us suppose that indeed the singularity is $\mathbb{Q}$-foliated Gorenstein then for some $m \in \mathbb{N}$ should we put, $u_{\alpha \beta}=\left(g_{\alpha \beta} h_{\beta \alpha}\right)^{m}$ and similarly for $\nu_{\alpha p}$ at the singularities then the corresponding cohomology class $\nu \in H^{1}\left(Z, \mathcal{O}_{Z}^{\times}\right)$must be trivial. For simplicity let us suppose that $Z$ has more than one component 
then the pull-back of $\nu$ to $H^{1}\left(Z_{i}, \mathcal{O}_{Z_{i}}^{\times}\right)$, for any $i$, is also trivial, and we denote the two singularities by $p$ and $q$. Further let's say that $Z_{i}$ in a neighbourhood whether of $p$ or $q$ is given by $x_{p}$ (resp. $x_{q}$ ) $=0$, then $\nu_{\alpha p}$ (resp. $\nu_{\alpha q}$ ) is equal to $b_{p}^{-m}\left(\right.$ resp. $\left.b_{q}^{-m}\right)$. Whence if the cohomology class $\nu$ is trivial over $Z_{i}$ there are functions $f_{\alpha}, f_{p}, f_{q}$ invertible on any $\Delta_{\alpha}, \Delta_{p}, \Delta_{q}$ such that $\nu_{\alpha \beta}=f_{\alpha} / f_{\beta}$ etc., so without loss of generality $f_{\alpha}=1$ for all $\alpha$, and $f_{p}=b_{p}^{m}$ and $f_{q}=b_{q}^{m}$. Now consider the other curve $Z_{j}$ through $p$ then since the various $f$ 's may be assumed to come from $Z$ we obtain in the same way $f_{p}=a_{p}^{m}$, and so the ratio $a_{p} / b_{p}(p)$ must be a root of unity. In the case of a rational curve with a node we deduce the same on considering the two branches through the singularity. At this point we choose a labelling of the singularities, of which there are $n$, and let $\lambda_{1}, \ldots, \lambda_{n}$ be the said ratios, or their inverses as appropriate, and apply the discussion of I.3 to obtain,

(a) $\sum_{i=1}^{n} Z_{i}^{2}=\sum_{i=1}^{n} \operatorname{Re}\left(\lambda_{i}+\frac{1}{\lambda_{i+1}}\right)$, if $n \geq 2$ and $\lambda_{n+1}:=\lambda_{1}$, or

(b) $-C^{2}+2=\operatorname{Re}\left(\lambda_{1}+1 / \lambda_{1}\right)$, if $n=1$.

In either case the right hand side has modulus bounded by $2 n$, which is strictly smaller than the modulus of the left since $Z$ is basic, whence the first part.

As for the second part, observe that $\Omega_{Y}^{1}(\log Z)$ is an extension of $K_{\mathcal{F}}$ by $N(Z)$ and the obstruction to splitting on a formal neighbourhood of $Z$ lies in,

$$
\underbrace{\lim }_{m} H^{1}\left(Z_{m}, N(Z) \otimes K_{\mathcal{F}}^{\vee}\right)=\underbrace{\lim }_{m} H^{1}\left(Z_{m}, K_{\mathcal{F}}^{-2}\right)
$$

where $Z_{m}$ is the $m^{\text {th }}$-thickening of $Z$. Since we know that $(X, \mathcal{F})$ is not foliated Gorenstein then $K_{\mathcal{F}}$ is not torsion so this obstruction certainly vanishes, i.e. on an infinitesimal neighbourhood of $Z$ we have a splitting,

$$
\Omega_{Y}^{1}(\log Z)=N(Z) \oplus K_{\mathcal{F}}
$$

Call the foliation defined by $N(Z), \mathcal{H}$. The transverse structure will permit us to conclude that we have a Hilbert-Modular surface. The question is purely local, so we might as well say that $Y$ is just its own completion in $Z$, or better an analytic neighbourhood since we want to consider its universal cover $\nu: \widetilde{Y} \rightarrow Y$. Necessarily $\pi_{1}(Y)=\pi_{1}(Z)=\mathbb{Z}$, and $\nu^{-1}(Z) \rightarrow Z$ the universal cover of $Z$ is just 
an infinite chain $C$ of rational curves. Now for any compact curve $C_{\alpha}$ in $C$ there is an open neighbourhood $U_{\alpha}$ in $\widetilde{Y}$ such that,

(a) The $U_{\alpha}$ cover $\tilde{Y}$ as $C_{\alpha}$ varies.

(b) The foliations $\mathcal{G}$ and $\mathcal{H}$ on $U_{\alpha}$ are defined by closed forms $\omega_{\alpha}$ and $\tau_{\alpha}$ respectively, with log-poles on $C$.

Indeed the latter question is local, and $C_{\alpha}$ is contracible to a Gorenstein canonical singularity for either of $\mathcal{G}$ or $\mathcal{H}$, so this follows from I.2.4 and the irrationality of the eigenvalues. On the other hand we can index the $C_{\alpha}$ so that both they and the $U_{\alpha}$ form an increasing chain, so that for $U_{\beta} \subset U_{\alpha}, \omega_{\beta}=g_{\alpha \beta} \omega_{\alpha}$ for some holomorphic transition functions $g_{\alpha \beta}$. The 1-forms in question are, however, closed so $g_{\alpha \beta}$ is invariant by $\mathcal{G}$, whence, since the intersection contains singularities of $\mathcal{G}$ is actually a constant. By construction such a topological co-cycle is a coboundary, so $\mathcal{G}$, and by an identical argument $\mathcal{H}$ are defined by closed holomorphic forms $\omega$ and $\tau$ on the whole of $\widetilde{Y}$ with log-poles along $C$, and we conclude by integrating $\omega$ and $\tau$ over $\widetilde{Y} \backslash C$.

From which it follows,

Corollary IV.2.3 Base point freeness for foliations of general type holds if and only if the foliation has no egl's. Moreover, foliations of general type with egl's exist, so base point freeness (equivalently finite generation of the canonical ring) for foliated surfaces of general type fails.

Proof The first assertion is clear by IV.2.2, so it remains to exhibit an example of a foliation of general type with an egl. To this end consider an egl. $\mathrm{Z}$ on a resolution $\pi:(Y, \mathcal{G}) \rightarrow(X, \mathcal{F})$ of the elliptic Gorenstein singularities of one of the natural foliations $\mathcal{F}$ on the Baily-Borel compactification of a Hilbert-Modular surface $X$. Here as in IV.2.2 we don't propose to resolve any quotient singularities on $Y$, so if one would like $Y$ to be globally smooth, its most expedient to introduce its Gorenstein covering stack $\mathcal{Y}$. Nevertheless $Y$ is smooth around $Z$, and since we'll be working exclusively around $Z$ we can reasonably assert that we have a splitting,

$$
\Omega_{Y}^{1}(\log Z)=N(Z) \oplus K_{\mathcal{F}}
$$


as before. Now denote by $\hat{Z}$ completion in $Z$, with, again, $Z_{m}$ the $m$ th infinitesimal thickening for the standard filtration, and $L:=K_{\mathcal{F}} \otimes N(Z)^{\vee}$.

With this in mind, observe that $\Gamma(\hat{Z}, L)$ is rather large. Indeed we have the standard exact sequence,

$$
\left.0 \longrightarrow L(-n Z)\right|_{Z} \longrightarrow L \otimes \mathcal{O}_{Z_{n+1}} \longrightarrow L \otimes \mathcal{O}_{Z_{n}} \longrightarrow 0
$$

for $n \in \mathbb{N}$. As such there is even a filtration, $\Gamma(\hat{Z}, L(-n Z)) \subset \Gamma(\hat{Z}, L)$ with successive quotients,

$$
\Gamma(Z, L(-n Z))
$$

which, in turn, has dimension $-n Z^{2}$. Thus, indeed, $\Gamma(\hat{Z}, L)$ is large, and,

$$
\Gamma(\hat{Z}, L)=\Gamma(\hat{Z}, L(-Z)) .
$$

Now choose any section $s$ of this latter group, and consider the foliation $\mathcal{F}_{s}$ on $\hat{Z}$ defined by,

$$
\operatorname{id} \oplus s: T_{\mathcal{F}} \longrightarrow T_{\hat{Z}}(-\log Z) .
$$

A check by local coordinates reveals that $Z$ is invariant by $\mathcal{F}_{s}$, and the singular locus, even scheme theoretically, of $\mathcal{F}_{s}$ is the same as that of $\mathcal{F}$, i.e. $Z$ is also an egl for $\mathcal{F}_{s}$. On the other hand by the theorem of formal functions, $\Gamma(\hat{Z}, L)$ is the completion at the contraction of $Z$ of $\pi_{*} L$, so we can certainly choose many $s$ which are meromorphic. Consequently with such a choice $\mathcal{F}_{s}$ extends to a foliation $\widetilde{\mathcal{F}}_{s}$, say, on $Y$ admitting $Z$ as an egl. It follows, however, from the classification theorem IV.5.11 that foliations which are not of general type with egl's are one of the natural foliations on a Hilbert-Modular surface. In particular, therefore, they have no moduli, whence for $s$ generic and algebraic, $\widetilde{\mathcal{F}}_{s}$ is of general type.

IV.3. Vectorial foliations $(\nu(X, \mathcal{F})=0)$

In this chapter we investigate foliations of numerical Kodaira dimension 0. Ultimately we'll see that up to an almost étale covering they are defined by global vector fields, but first we'll need to take care of irregular surfaces, i.e.

Lemma IV.3.1. Let $(X, \mathcal{F})$ be a model of a foliated surface with canonical singularities and $\nu(X, \mathcal{F})=0$, then abundance holds. Better still if $X$ is smooth and irregular then in fact $h^{0}\left(K_{\mathcal{F}}\right) \neq 0$ or the foliation is a fibration. 
Proof. The lemma is trivial for surfaces with zero irregularity, so suppose otherwise and without loss of generality let $(X, \mathcal{F})$ be the minimal desingularisation of a canonical model, then we have an exact sequence,

$$
0 \rightarrow N \rightarrow \Omega_{X}^{1} \rightarrow K_{\mathcal{F}} \cdot I_{Z} \rightarrow 0
$$

giving rise to the foliation. Observe that by a classical lemma of Castelnuovo should $h^{0}(X, N) \geq 2$ then the foliation is a fibration, while if $q(X)=1$ then $h^{0}\left(K_{\mathcal{F}}\right) \neq 0$ or the foliation is the Albanese fibration. Whence we may suppose that the quotient $\pi: X \rightarrow X / \mathcal{F}:=B$ exists and factors through the Albanese. Better still the foliation is certainly an elliptic fibration, so by Kodaira's canonical bundle formula we obtain,

$$
K_{\mathcal{F}}=\pi^{*}\left\{\left(R^{1} \pi_{*} \mathcal{O}_{X}\right)^{\vee}\right\}\left(-\sum_{i}\left(D_{i}\right)\right)
$$

the sum being taken over all fibres $F_{i}$ such that the underlying space $\left(F_{i}\right)_{\text {red }}$ is singular and $D_{i}$ is a proper effective Cartier divisor contained in $F_{i}$. To profit from this observation observe that by III.3.2 and the fact that $\pi$ factors through the Albanese there is a $\mathbb{Q}$-Cartier divisor $L$ of degree 0 on $B$, and $\mathbb{Q}$-Cartier divisors $Z_{i j}$ supported on -1-chains inside $F_{i}$ such that,

$$
K_{\mathcal{F}}=\pi^{*} L+\sum_{i j} Z_{i j}
$$

as $\mathbb{Q}$-Cartier divisors. Consequently if $p:(X, \mathcal{F}) \rightarrow\left(X_{0}, \mathcal{F}_{0}\right)$ is a contraction of the -1 -chains then, $p_{*}\left(K_{\mathcal{F}} \otimes \pi^{*} L^{\vee}\right)$ is torsion and $\pi$ descends to $X_{0}$. We may thus take a cover $q:\left(Y_{0}, \mathcal{G}_{0}\right) \rightarrow\left(X_{0}, \mathcal{F}_{0}\right)$ which is étale in codimension one and such that $K_{\mathcal{G}_{0}}$ is the pull-back of a Cartier divisor on $B$ which is numerically equivalent to zero. Now putting $p:(Y, \mathcal{G}) \rightarrow\left(Y_{0}, \mathcal{G}_{0}\right)$ to be a minimal desingularisation, profiting from III.1.4, and essentially the arguments of III.3 (although see IV.3.3 et sequel) we see that $K_{\mathcal{G}}$ is numerically equivalent to zero as a Cartier divisor. Nevertheless the foliation is still an elliptic fibration so applying the previous formula for $K_{\mathcal{F}}$ but now to $K_{\mathcal{G}}$ we see that $D_{i}$ must be a multiple of $F_{i}$ by Zariski's lemma, so it is $F_{i}$ and so continuing to denote the quotient by $\pi$ we have, $\operatorname{deg}\left(R^{1} \pi_{*} \mathcal{O}_{Y}\right)^{\vee}=0$. Consequently the only multiple fibres of $\pi: Y \rightarrow Y / \mathcal{G}$ are non-singular elliptic curves so the foliation is smooth on $Y$ (otherwise there 
would be a curve intersecting $K_{\mathcal{G}}$ positively). Appealing to [BPV] (III.18.3) we deduce that $K_{\mathcal{F}}$ is torsion.

Divertimento IV.3.1 $\mathbf{1}^{\mathbf{1} \mathbf{2}}$. Obviously what we've done in the course of the proof is under the hypothesis of positive irregularity with $K_{\mathcal{F}}$ not inheriting a global section from the space of 1-forms is to construct an almost étale cover $q:(Y, \mathcal{G}) \rightarrow$ $\left(X_{0}, \mathcal{F}_{0}\right)$ with $Y$ non-singular and $\pi: Y \rightarrow Y / \mathcal{G}$ an elliptic fibration with $R^{1} \pi_{*} \mathcal{O}_{Y}$ torsion. Now if $Y / \mathcal{G}$ has positive genus then it's a theorem (cf. [R] E.5.1) that $Y$ is a sesquielliptic surface, i.e. $Y=C \times E / G$ where $G$ is an abelian group acting on an elliptic curve $E$ by translations, on $C$ any old how, and the diagonal action is free. The proof however has no need of this further hypothesis, so putting $Y / \mathcal{G}=B$ let's give the details since they will arise often enough. The important point is that Kodaira's canonical bundle formula gives, $q=p_{g}+1 \geq g(B)+1$. Consequently if $\alpha: Y \rightarrow \operatorname{Alb}(Y)$ is the Albanese, then $\alpha$ cannot factor through $B$, and $\alpha \times \pi: Y \rightarrow \operatorname{Alb}(Y) \times B$ expresses $Y$ as a finite cover of a family of elliptic curves in a fixed abelian variety. Such a family is necessarily constant, so in fact for some elliptic curve $A$ which is a quotient of $\operatorname{Alb}(Y)$ every fibre $Y_{b}$ admits a non-constant map to $A$, which in fact is Galois with abelian group, and the normalised fibre product $Y \times_{B} A$ is a product as required. Consequently we'll use the sesquielliptic terminology even for a base of genus 0 , and when we come across this case again we'll explicitly note it in the classification.

We now change our notation slightly and denote by $\left(X^{\prime}, \mathcal{F}^{\prime}\right)$ the unique minimal desingularisation of the canonical model of a foliated surface $(X, \mathcal{F})$. From IV.3.1 and $\nu(X, \mathcal{F})=0$ we deduce that $(X, \mathcal{F})$ is in fact $\mathbb{Q}$-foliated Gorenstein, whence there are no e.g.l.'s. To advance further, we observe,

Lemma IV.3.2. Let $(Y, \mathcal{G})$ be a foliated surface with $K_{\mathcal{G}}$ isomorphic to the structure sheaf in codimension 2, then in fact $(Y, \mathcal{G})$ is foliated Gorenstein and indeed $K_{\mathcal{G}}$ is not just the structure sheaf, but defines an element of $H^{0}\left(\mathcal{T}_{Y}\right)$.

Proof. Recall that by definition $K_{\mathcal{G}}$ is the dual of a saturated subsheaf of $\mathcal{T}_{Y}$, so that this is just an easy exercise in local cohomology, cf. [M1], 2.3.

To apply this, we extract the $n$th root, $Y$, of a globally trivialising section of $K_{\mathcal{F}}^{\otimes n}$, with $n$ minimal, as guaranteed by IV.3.1, so on appealing to I.1.12,

Fact IV.3.3. Let $(X, \mathcal{F})$ be a canonical model of a foliation with $\nu(X, \mathcal{F})=0$, 
then there is a cyclic Kummer covering, $p:(Y, \mathcal{G}) \rightarrow(X, \mathcal{F})$ étale in codimension one with canonical singularities defined by a global vector field.

Let us therefore consider the problem of a foliated surface $(Y, \mathcal{G})$ with canonical singularities defined by a saturated global vector field, and put $G$ to be the connected component of the identity in $\operatorname{Aut}(Y)$ so that,

Fact IV.3.4. Notations as above, $T_{1} G=H^{0}\left(\mathcal{T}_{Y}\right)$.

Also, for any $y \in Y$, we have a map $f_{y}: G \rightarrow Y: g \mapsto y^{g}$, whose differential $d f_{y}: T_{1} G \rightarrow T_{y} Y$ is the image of this identification restricted to $H^{0}\left(\mathcal{T}_{Y}\right)$ in $T_{y} Y$.

As to the singularities of the surface $Y$, these are necessarily fixed points of $G$, so that if $p:\left(Y^{\prime}, \mathcal{G}^{\prime}\right) \rightarrow(Y, \mathcal{G})$ is a minimal resolution of singularities then $Y^{\prime}$ is $G$-equivariant. Moreover as in IV.3.1 essentially the same arguments as in III.3 shows that $K_{\mathcal{G}^{\prime}}=\rho^{*} K_{\mathcal{G}}+E$, where $E$ is a divisor supported on -1-chains which is not integral. However $(Y, \mathcal{G})$ is foliated Gorenstein so $E$ must be zero by III.1.4. Now consider the problem of curves $E$ in $Y^{\prime}$ with $E^{2}=-1$. By adjunction these are invariant for any vector field, so they aren't just invariant for our foliation $\mathcal{G}$ but the whole group $G$. Since $K_{\mathcal{G}} \stackrel{\sim}{\rightarrow} \mathcal{O}_{Y}$, they can be blown down with discrepancy 0 , and so we obtain a minimal surface $S$ blown down from $Y$ together with a foliation $\mathcal{H}$. The surface $S$ is $G$-equivariant, $(S, \mathcal{H})$ has canonical singularities, and $K_{\mathcal{H}} \stackrel{\sim}{\rightarrow} \mathcal{O}_{S}$.

Firstly consider the case that $G$ is not transitive, then the general integral sub-curve is rational or elliptic. By IV.1.1 the former is impossible. As ever the quotient space $B=S / \mathcal{H}$ exists and is a regular curve, and the quotient map $\pi: S \rightarrow B$ is an elliptic fibration. In addition as in IV.3.1,

$$
\mathcal{O}_{S} \stackrel{\sim}{\longleftarrow} K_{\mathcal{H}} \stackrel{\sim}{\longrightarrow}\left(R^{1} \pi_{*} \mathcal{O}_{S}\right)^{\vee}
$$

and every fibre is thus an elliptic curve, so arguing as in op.cit., we deduce that $\operatorname{sing}(\mathcal{H})=\emptyset$, so $c_{1}^{2}(S)=c_{2}(S)=0$, and $p_{g}(S) \geq g(B)$, the genus of $B$. Necessarily then, $q(S) \geq g(B)+1$, so that an étale cover of $S$ is a product $E \times B$ for some fixed elliptic curve $E$. Indeed more precisely we have,

Case IV.3.5 (a) $(Y, \mathcal{G})$ is the natural elliptic fibration on a sesquielliptic surface (cf. IV.3.1 $1^{1 / 2}$ ), so essentially just $E \times B$ for $E$ an elliptic curve and $B$ arbitrary, and the canonical model is unique. 
Before continuing observe that this certainly follows from the above discussion, since $(S, \mathcal{H})$ regular and $K_{\mathcal{G}^{\prime}} \stackrel{\sim}{\longrightarrow} \mathcal{O}_{Y^{\prime}}$ forces $S=Y^{\prime}$. Further $Y^{\prime}$ contains no $-2 F$-curves so by III.3.3, $Y^{\prime}=Y$.

So suppose that the action of $G$ is transitive. The easiest case is if $G$ is compact, then $S$ is an abelian surface. Thus we either have a fibration by elliptic curves, and in particular IV.3.5 (a) above, or $(S, \mathcal{H})$ is a Kronecker foliation, i.e. defined by a global holomorphic one form $\omega$ which is necessarily some combination $\lambda \omega_{0}+\mu \omega_{1}$ of a basis of $H^{0}\left(S, \Omega_{S}^{1}\right)$ in such a way that no leaf is compact. Similar considerations to the remarks following the above case, ensure that this case reduces to,

Case IV.3.5 (b) $(Y, \mathcal{G})$ is a Kronecker foliation on an abelian surface, and the canonical model is unique.

Otherwise $G$ is an extension of an abelian variety $A$, say, by an affine linear group $H$, and we next consider the possibility that $A \neq 0$ but $G$ is not compact. Choose a general point $s \in S$ and let $N$ be the normaliser of the stabiliser of $s$ in $G$ then as in IV.3.4 we have a map, but now injective,

$$
f_{s}: G / N \hookrightarrow S
$$

Since $S$ is minimal, it is therefore the natural compactification of an extension of an elliptic curve $E$ by $\mathbb{G}_{a}$ or $\mathbb{G}_{m}$, obtained by adding the section at infinity or sections at zero and infinity. Let $\pi: S \rightarrow E$ be the natural map. The foliation cannot be a fibration by rational curves and so the map,

$$
\mathcal{O}_{S} \stackrel{\sim}{\longleftarrow} \pi^{*} \omega_{E} \longrightarrow K_{\mathcal{H}} I_{\operatorname{sing}(\mathcal{H})} \stackrel{\sim}{\longleftarrow} I_{\operatorname{sing}(\mathcal{H})}
$$

is non-zero, whence necessarily the foliation is without singularities, and either $S=\mathbb{P}^{1} \times E$ or the connected component of $\operatorname{Aut}(S)$ is the said extension. In either case we have a splitting,

$$
T_{S}=T_{S / E} \oplus \pi^{*} T_{E}
$$

and the foliation is everywhere transverse to the fibration over $E$. This excludes the possibility of rational integral sub-curves and forces the identification $(S, \mathcal{H})=$ $(Y, \mathcal{G})$. In addition even if $S$ is split we still have natural sections at zero and infinity (respectively $\infty$ ) given by the zero locus of the $\mathbb{P}^{1}$-part of the vector field, 
and the structure group naturally reduces to $E \times \mathbb{G}_{m}$ (respectively $E \times \mathbb{G}_{a}$ ) and so we obtain,

Case IV.3.5 (c) The necessarily unique canonical model $(Y, \mathcal{G})$ is either,

(i) An elliptic vector foliation, i.e. $Y$ is the natural equivariant compactification of an extension of $E$ by $\mathbb{G}_{a}$, and the foliation is defined by a vector field everywhere transverse to the natural fibration by rational curves, with a unique compact leaf, the natural section at infinity.

(ii) A semi-abelian foliation, i.e. $Y$ is the natural equivariant compactification of an extension of $E$ by $\mathbb{G}_{m}$, the foliation is defined by a vector field everywhere transverse to the natural fibration by rational curves, and there are two compact leaves, the natural sections at zero and infinity.

We are thus left with the possibility that the group $G$ is affine and acting transitively on $S$, so $S$ is rational. For the moment we ignore the possibility that $S$ is $\mathbb{P}^{2}$ or $\mathbb{P}^{1} \times \mathbb{P}^{1}$ and consider only the cases that $\pi: S=\mathbb{P}\left(\mathbb{I} \oplus H^{\otimes n}\right) \rightarrow \mathbb{P}^{1}$, is a Hizerbruch surface, $H$ the tautological bundle on $\mathbb{P}^{1}, n \geq 2$. The necessary generality is however maintained by surreptitiously allowing the possibility that $n=0$ or 1 . In any case $c_{2}(S)=4$, so if $Z$ is the singular locus of $\mathcal{H}$ then $\operatorname{deg} Z$, counting nilpotents, is 4 (if $S=\mathbb{P}^{2}$, then $\operatorname{deg} Z=3$, choose a canonical singularity and blow up in its reduced structure to get the case $n=1$, and maintain $K_{\mathcal{H}}$ trivial). For any fibre $F$ of $\pi$, we have $K_{\mathcal{H}} \cdot F=F^{2}=0$. However if $F$ contains a singular point of $\mathcal{H}$ and $F$ is not an integral sub-curve then $\left(K_{\mathcal{H}}+F\right) \cdot F>0$, so a fibre containing a singularity is an integral sub-curve.

Consequently if we're on $\mathbb{P}^{1} \times \mathbb{P}^{1}$ the situation is nice and clear. Either there are no saddle nodes and for each direction we have two invariant fibres, giving an invariant boundary $\partial$ consisting of 4 fibres with the foliation $\mathcal{H}$ defined by an injection of bundles,

$$
\mathcal{O}_{S} \hookrightarrow T_{S}(\log \partial) \stackrel{\sim}{\longrightarrow} \mathcal{O}_{S} \oplus \mathcal{O}_{S}
$$

and since it's not a fibration by rational curves it must be a Kronecker type foliation (i.e. in standard coordinates given by $\frac{d x}{x}+\lambda \frac{d y}{y}, \lambda \notin \mathbb{Q}$ ) and everything is governed by the toric action, i.e. the group in question is $\mathbb{G}_{m} \times \mathbb{G}_{m}$. Otherwise there is a saddle node, and indeed by a trivial explicit calculation two saddle nodes, leading to two invariant fibres in one direction, each of which is the weak 
branch of the node, and one invariant fibre in the other direction which passes through both singular points. In particular we have an invariant boundary $\partial$ consisting of 3 invariant curves, in a suitable standard coordinate system the vector field in question is $x^{2} \frac{\partial}{\partial x}+\lambda y \frac{\partial}{\partial y}, \lambda \in \mathbb{C}$, and the group in question is $\mathbb{G}_{a} \times \mathbb{G}_{m}$.

Evidently we wish to obtain the same conclusion in the general case $n \geq 1$. Here there is a canonical section $C$ of $\pi$ with $C^{2}<0$, which by adjunction is invariant. There is of course an invariant fibre $F$, meeting $C$ in a singular point $z$, and if the singular locus of $\mathcal{H}$ along $F$ has Segre class 1 at $z$, then there must be another singularity $w$ on $F$. We can blow up in $w$, and blow down $F$ without changing any of our hypothesis except that $n$ decreases by 1 , and so if this situation persists reduce to the $\mathbb{P}^{1} \times \mathbb{P}^{1}$, i.e. $n=0$, situation. From which we deduce that we have at least 3 invariant curves, and a $\mathbb{G}_{m} \times \mathbb{G}_{m}$ or $\mathbb{G}_{a} \times \mathbb{G}_{m}$ action according to whether we have a $4^{\text {th }}$ or not. The tricky case is therefore if the Segre class of $\mathcal{H}$ along $F$ is 2, i.e. $F$ is a weak branch of a node. This implies that we have another singularity $w$ on $C$, and we might as well say $\pi(z)=0$, $\pi(w)=\infty$ in standard coordinates on $\mathbb{P}^{1}$, so at $z$ we can write the global vector field defining $\mathcal{H}$ as,

$$
\partial=x \frac{\partial}{\partial x}+g(x)\left(y^{2} \frac{\partial}{\partial y}\right)
$$

for $y$ the natural coordinate for the contractible section of a Hizerbruch surface. If $\xi=1 / x$ then in the transverse coordinate, $\eta=x^{n} y$, at $\infty$,

$$
\partial=-\xi \frac{\partial}{\partial \xi}+n \eta \frac{\partial}{\partial \eta}+\left(g\left(\frac{1}{\xi}\right) \xi^{n}\right) \eta^{2} \frac{\partial}{\partial \eta} .
$$

So apart from the obvious fact that $g$ has degree at most $n$, since $n \neq 0$ there must be a second singularity on the fibre at $\infty$, i.e. it cannot also be the weak branch of a node. Whence if there is only one singularity on $F$, we can find another invariant fibre $F^{\prime}$ on which there are two singularities, blow up in the one off $C$, and blow down to reduce $n$, thus arriving in the $\mathbb{P}^{1} \times \mathbb{P}^{1}$ situation. From which we can note the final two cases of the classification,

Case IV.3.5 (d) (i) $(Y, \mathcal{G})$ is a Kronecker foliation on an absolutely minimal toric variety. In this case the canonical model is not unique, and may be flipped in its birational equivalence class. 
We justify this deduction as follows. Necessarily $\left(Y^{\prime}, \mathcal{G}^{\prime}\right)$ is obtained by sequentially blowing up $(S, \mathcal{H})$ in zero dimensional strata of the $\mathbb{G}_{m} \times \mathbb{G}_{m}$ action, and $(Y, \mathcal{G})$ is obtained by sequentially blowing down 1-dimensional strata. Consequently $(Y, \mathcal{G})$ is smooth outside of zero dimensional strata. Now it suffices for the argumentation that has lead us to deduce the nature of $(S, \mathcal{H})$ that $(X, \mathcal{F})$ contained no -1-chains, so suppose it is such a contraction of $\left(X^{\prime}, \mathcal{F}^{\prime}\right)$ rather than the actual canonical model, which in turn we denote by $\left(X_{0}, \mathcal{F}_{0}\right)$, and $\left(Y_{0}, \mathcal{G}_{0}\right)$ the corresponding cover, then any exceptional divisor whose centre lies over a singularity of $(X, \mathcal{F})$ has negative discrepancy. However any exceptional divisor over the pre-image of a singularity in $(Y, \mathcal{G})$ is such a divisor so that by IV.3.3 (or more precisely its proof) it must have negative discrepancy. Equally by virtue of our explicit description of $(Y, \mathcal{G})$ the discrepancy for the exceptional divisor over a zero dimensional stratum is zero, so the covering map is actually étale in a neighbourhood of the same. Moreover if an irreducible curve of the 1-dimensional stratum contained a point over a singular point of $(X, \mathcal{F})$ then the image of such a curve in $(X, \mathcal{F})$ meets the singular locus of the foliation in 2-points on the nonsingular locus of $X$ and also passes through one singular point of the underlying space, and so by the considerations of III.3 $K_{\mathcal{F}}$ has positive intersection with such a curve, which is absurd. Consequently if $T=\sum C_{i}$ is a chain of rational integrable sub-curves on $X$ contracted by the canonical model procedure, then the cover is actually étale in a neighbourhood of $T$. Whence if $d$ is the degree of the cover then the pre-image of any $C_{i}$ is $d$ disjoint rational curves, $C_{i j}$ say, with $C_{i j}^{2}=C_{i}$. Further for reasons of counting singularities the pre-image of $T$ is thus a disjoint union of $d$-chains of contractible rational integrable sub-curves, and the cover $\left(Y_{0}, \mathcal{G}_{0}\right)$ of $\left(X_{0}, \mathcal{F}_{0}\right)$ is itself a canonical model as required.

Arguing similarly we obtain,

Case IV.3.5 (d) (ii) $(Y, \mathcal{G})$ is a toric vector foliation, i.e. a foliation of the above form on an absolutely minimal compactification of $\mathbb{G}_{a} \times \mathbb{G}_{m}$ (i.e. no component of the boundary may be contracted).

Apart from these final cases, one doesn't get an enormous simplification of this discussion by appealing to stacks, but there is some elegant clarification of the geometry. In particular, if we introduce the Gorenstein covering stack $(\mathcal{X}, \mathcal{F}) \rightarrow(X, \mathcal{F})$ of the canonical model, then, by definition $p: Y \rightarrow X$ of 
IV.3.3 factors through $\mathcal{X}$, and $Y \rightarrow \mathcal{X}$ is everywhere étale. In particular, $(Y, \mathcal{G})$ must necessarily satisfy III.3.1, so that by I.1.12, it's a canonical model defined by a global vector field, and $\mathcal{X}$ is a classifying stack of the form $[Y / C]$, for $C$ a cyclic group preserving the vector field. Furthermore, the minimal resolution $Y^{\prime} \rightarrow Y$ is unique, so $C$ equivariant, and, as before, contains no curves of square -1 , while $Y$ contains no contractible curves whatsoever. Whence if contracted Hizerbruch denotes a Hizerbruch surface on which the unique contractible curve is contracted, we may summarise by way of,

Theorem 2 IV.3.6. The Gorenstein covering stack of a canonical model of a foliation by curves of numerical Kodaira dimension 0 is the fine moduli of the quotient by a finite cyclic group $C$ acting without pseudo reflections (so, in particular, the model itself is the course moduli) which preserves the foliation on any of the following,

\begin{tabular}{|c|c|c|}
\hline Underlying space $Y$ & $\begin{array}{c}\text { Group of } \\
\text { automorphisms }\end{array}$ & $\begin{array}{c}\text { Element of } \\
\text { the Lie algebra }\end{array}$ \\
\hline Sesquielliptic surface & An elliptic curve & Any generator \\
\hline Abelian surface & Abelian surface & $\begin{array}{l}\text { Any irrational field, } \\
\text { i.e. not coming from } \\
\text { an elliptic fibration }\end{array}$ \\
\hline $\begin{array}{l}\text { Compactification of a } \\
\text { semi-abelian }\end{array}$ & Semi-abelian surface & $\begin{array}{l}\text { Any generator whose } \\
\text { projections to either }\end{array}$ \\
\hline $\begin{array}{l}\text { surface as a } \mathbb{P}^{1} \text {-bundle } \\
\text { over an elliptic curve by }\end{array}$ & & factor is non-trivial \\
\hline adding sections at 0 and $\infty$ & & \\
\hline $\begin{array}{l}\text { Idem, but now a compact- } \\
\text { ification of an extension of } \\
E \text { by } \mathbb{G}_{a} \text { by section at } \infty\end{array}$ & $\begin{array}{l}\text { Extension of } \\
\text { an elliptic curve by } \mathbb{G}_{a}\end{array}$ & Idem \\
\hline $\begin{array}{l}\text { Contracted Hizerbruch, } \\
\qquad \mathbb{P}^{2}, \mathbb{P}^{1} \times \mathbb{P}^{1}\end{array}$ & $\mathbb{G}_{m} \times \mathbb{G}_{a}$ & Idem \\
\hline $\begin{array}{l}\text { Contracted Hizerbruch, } \\
\qquad \mathbb{P}^{2}, \mathbb{P}^{1} \times \mathbb{P}^{1}\end{array}$ & $\mathbb{G}_{m} \times \mathbb{G}_{m}$ & $\begin{array}{l}\text { Any irrational field, } \\
x \frac{\partial}{\partial x}+\lambda y \frac{\partial}{\partial y}, \lambda \notin \mathbb{Q}\end{array}$ \\
\hline
\end{tabular}

Plainly, the models is unique within its birational equivalence class except 
more or less by definition in the cases governed by $\mathbb{G}_{m} \times \mathbb{G}_{a}$ or $\mathbb{G}_{m} \times \mathbb{G}_{m}$ which as indicated may be arbitrary contracted Hizerbruch surfaces. Needless to say, one can identify the group of automorphisms $C$, and this has been done, $[\mathrm{P}]$. Indeed, the essential is to note that on the universal cover everything is linear, so that $C$ must be a cyclic sub-group of $\mathrm{GL}\left(\mathbb{Z}^{\oplus n}\right)$, for $1 \leq n \leq 4$. This implies, cf. op. cit., that in the abelian case the cardinality of $C$ can be anything up to 12 except 7, or 9 , while in the penultimate case it is 1 or 2 , and in all other cases it's anything up to 6 except 5 . All of these possibilities, in all of the individual cases can occur. As such, IV.3.6 is rather optimal, and doesn't leave much room for,

Generalisations IV.3.7. In the 2 category of algebraic stacks, there are no essentially new features, and this continues to be true (excepting issues of rationality) if we even allow log-canonical singularities. Indeed the only new feature arises when we permit the possibility of boundaries of weight 1 , which gives rise to a 'sesquitoric' case, i.e. in the sesquielliptic case we replace elliptic curves by $\mathbb{G}_{m}$ 's.

\section{IV.4. Kodaira dimension 1}

In this section we study foliations whose Kodaira dimension $\kappa(X, \mathcal{F})$, as opposed to its numerical Kodaira dimension, is equal to 1 . We begin with,

Lemma IV.4.1. Let $(X, \mathcal{F})$ be a foliated smooth surface with canonical singularities, and $\kappa(X, \mathcal{F}) 0$ or 1 , then it has no egl's.

Proof. By hypothesis some power of $K_{\mathcal{F}}$, say $K_{\mathcal{F}}^{\otimes m}$, has a global section $\omega$, and there exists some egl with fundamental cycle $Z$. Consider the cases:

(a) $\omega$ does not vanish on any component of $Z$.

In consequence if $\widehat{X}$ is the completion of $X$ along $Z$ then $K_{\mathcal{F}}^{\otimes m} \mid \widehat{X}$ has a nowhere vanishing section, and we're done by IV.2.2.

(b) $\omega$ vanishes on some components of $Z$.

In this case we write $\operatorname{div}(\omega)=D+W$, where $W$ is supported on $Z$ and the curves in the support of $D$ are distinct from $Z$. Moreover we can make this decomposition not just on $(X, \mathcal{F})$ but on $(Y, \mathcal{G})$, say, where $(Y, \mathcal{G})$ is obtained by contracting the rational singularities of $A_{n}$ or $D_{n}$ type so that $K_{\mathcal{G}}$ is nef. The 
Kodaira dimension is however less than 2 so we obtain, $K_{\mathcal{G}} . D=0$. Whence if $C$ is a curve in the support of $D$ then $C^{2} \geq 0$. Bearing in mind once again that $K_{\mathcal{G}}$ is not big we obtain in addition that $C^{2}=D^{2}=D$. $C=0$, and whence $W$. $D=0$. Necessarily then $K_{\mathcal{G}}^{2}=W^{2}<0$, which is absurd.

An immediate application of the lemma and III.3.2 gives,

Corollary IV.4.2. A canonical model with $\kappa(X, \mathcal{F})=1$ is $\mathbb{Q}$-factorial.

Now let us concentrate on canonical foliated surfaces $(X, \mathcal{F})$ of Kodaira dimension 1 , and let $\rho:(\widetilde{X}, \widetilde{\mathcal{F}}) \rightarrow(X, \mathcal{F})$ be the unique minimal resolution of the underlying space. In addition let two sections of $K_{\mathcal{F}}^{\otimes m}$ be given with underlying divisors $D=\sum_{i} a_{i} C_{i}$ and $D^{\prime}=\sum_{i} a_{i}^{\prime} C_{i}^{\prime}$ then, $C_{i} \cdot C_{j}=C_{i} \cdot C_{j}^{\prime}=C_{i}^{\prime}$. $C_{j}^{\prime}=0$, for all possible combinations of $i$ and $j$. On the other hand, we can certainly find an effective divisor $E$ and sub-divisors $D_{0}, D_{0}^{\prime}$ of $D$ and $D^{\prime}$ respectively such that,

$$
m K_{\mathcal{F}}-E=D_{0}=D_{0}^{\prime}
$$

and since $D_{0} \cdot D_{0}^{\prime}=0$ this implies that $\left|m K_{\mathcal{F}}-E\right|$ defines a base point free pencil. Naturally we make,

Definition/Observation IV.4.3 $\mathrm{L} \pi:(Y, \mathcal{G}) \rightarrow(X, \mathcal{F})$ a resolution with underlying space $Y$ smoothet $\pi: X \rightarrow B$ be its Stein factorisation, then the above considerations imply that every fibre is an irreducible curve, and for any $b \in B$, $X_{b} . E=0$, so $E$ itself is contained in a fibre, and whence there is an effective $\mathbb{Q}$-Cartier divisor $K$ on $B$ such that $K_{\mathcal{F}}=\pi^{*} K$.

Let us proceed immediately to the case where the foliation is a pencil. Manifestly the generic fibre $X_{b}$ is an elliptic curve. By Kodaira's canonical bundle formula any elliptic fibration has foliated Kodaira dimension at most 1, and by IV.3 at least one, unless after an almost étale cover the fibration is a product so we obtain,

Fact IV.4.4(i) If the foliation is a pencil, then it is an elliptic fibration which is not an elliptic fibration of product type after an almost étale cover.

At this point, it's convenient to consider Kodaira's tabulation of singular elliptic fibres from the foliated point of view. Specifically,

Proposition IV.4.5. With hypothesis as given the unique minimal desingulari- 
sation $\rho:(\widetilde{X}, \widetilde{\mathcal{F}}) \rightarrow(X, \mathcal{F})$ of a canonical foliated surface of Kodaira dimension 1 is an isomorphism in a neighbourhood of an invariant fibre unless the fibration $\pi: X \rightarrow B$ is an elliptic fibration, or the fibre is the special invariant rational fibre described at the end of the proof.

Proof. In light of the classification of the singularities of $X$ there are only 3 possibilities for a singular fibre which meets an integral sub-curve, viz: -1-chains, a chain of $-2 F$-curves, or $D_{n}{ }^{2}$. Let us for the moment call this information of types I, II and III respectively and let $\widetilde{C}$ in $\widetilde{X}$ denote the proper transform of a fibre $C$ through which there is a singularity. Consequently if $T_{1}, \ldots, T_{k}$ are the maximal -1-chains in $\widetilde{X}$ meeting $\widetilde{C}$ with discriminants $d_{1}, \ldots, d_{k}$ of the corresponding intersection matrices normalised in a positive sense then as ever $s_{\mathrm{I}} \geq k$ and $d_{i} \geq 2, \forall 1 \leq i \leq k$. Furthermore we have the formula,

$$
K_{\mathcal{F}} \cdot C=(2 g-2)+s_{0}+s_{\mathrm{I}}+s_{\mathrm{II}}+s_{\mathrm{III}}-\sum_{i=1}^{k} \frac{1}{d_{i}}
$$

where $s_{\mathrm{I}}, s_{\mathrm{II}}, s_{\mathrm{III}}$ are the multiplicities of the Segre class of $C$ along the singular locus of $\widetilde{\mathcal{F}}$ supported at the type I, II and III information respectively, $s_{0}$ the Segre class at any singularities of $\mathcal{F}$ outside of these, and of course $g$ is the genus of $C$. Consequently if $k>0$ then certainly $g=0$, while if $k=0$ then $g \leq 1$ and indeed $g=1$ would imply that $s_{\mathrm{II}}+s_{\mathrm{III}}=0$. This being absurd, we conclude that $C$ is always of genus 0 , and the above formula becomes,

$$
2+\sum_{i=1}^{k} \frac{1}{d_{i}}=s_{0}+s_{\mathrm{I}}+s_{\mathrm{II}}+s_{\mathrm{III}}
$$

and we simply tabulate and consider all possibilities. Necessarily $k \leq 4, s_{\mathrm{I}}+s_{\mathrm{II}}+$

\footnotetext{
${ }^{2}$ We abuse standard convention, and for obvious reasons consider a " $D_{3}$ " where the central node has valency 3 , with an edge towards a possibly inexistent node, corresponding to a foliation singularity.
} 
$s_{\text {III }} \geq 1$ and we have:

$\begin{array}{ccccc}k & s_{0} & s_{\mathrm{I}} & s_{\mathrm{II}} & s_{\mathrm{III}} \\ 0 & 1 & 0 & 1 & 0 \\ 0 & 1 & 0 & 0 & 1 \\ 0 & 0 & 0 & 1 & 1 \\ 0 & 0 & 0 & 2 & 0 \\ 0 & 0 & 0 & 0 & 2 \\ 2 & 0 & 3 & 0 & 0 \\ 2 & 1 & 2 & 0 & 0 \\ 2 & 0 & 2 & 1 & 0 \\ 2 & 0 & 2 & 0 & 1 \\ 3 & 0 & 3 & 0 & 0 \\ 4 & 0 & 4 & 0 & 0\end{array}$

Naturally we begin with $k=0$, so that $\widetilde{C}$ must be a $-2 F$-curve, whence by the construction of the canonical model $\widetilde{C}^{2} \leq-2$. On the other hand the intersection matrix of the curves in $p^{-1}(C)$ must be indefinite so we may apply the classification of integral quadratic forms to conclude that the said intersection matrix is one of the forms,

$$
\bar{A}_{n}, n+1 \text { vertices, } n \geq 1
$$
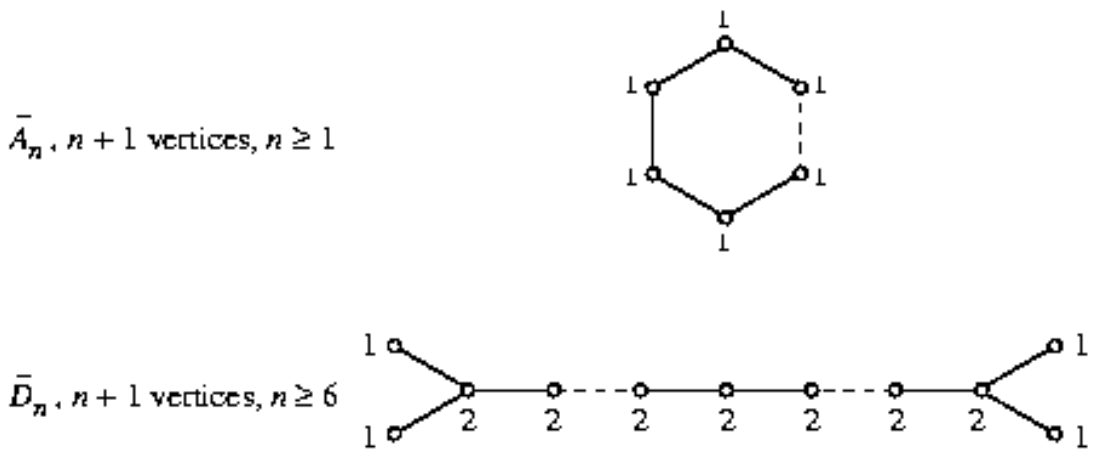

arising from the cases $s_{\mathrm{II}}=2, s_{\mathrm{III}}=0$ and $s_{\mathrm{II}}=0, s_{\mathrm{III}}=2$ respectively. In addition the fact that $C^{2}=0$ obliges all the nodes to correspond to curves with self-intersection -2 (i.e. this really is the usual Dynkin diagram rather than 
the dual graph of the fibre) and the corresponding fibre over $b=\pi(c)$ to be a multiple of the corresponding root system as indicated. Necessarily then the euler characteristic of the fibre $\widetilde{X}_{b}$ is zero as required.

Now let us turn to the case $k=2$. The sub-cases $s_{\mathrm{I}}=3$ and $s_{0}=1$ are a little awkward, and we will return to them at the end. Regardless we must have $d_{1}=d_{2}=2$, and so for $s_{\mathrm{I}}=2$ the hypothesis that $C^{2}=0$ forces $\widetilde{C}^{2} \geq-2$, and permits us to argue as above to conclude that the only possibility for the fibre $\widetilde{X}_{b}$ is $\widetilde{D}_{n}, n \geq 5$, corresponding to the case $s_{\text {III }}=1$, and that once again $\pi$ is an elliptic fibration.

Next we take up the case $k=3$, where we have the possible arrangements for the discriminants $\left(d_{1}, d_{2}, d_{3}\right)$ being $(2,3,6),(2,4,4)$ and $(3,3,3)$ respectively. Now if we consider the case $\widetilde{C}^{2} \leqq-2$ then the various integrability conditions impose that the fibre must be a multiple of the root system in the respective Dynkin diagrams,
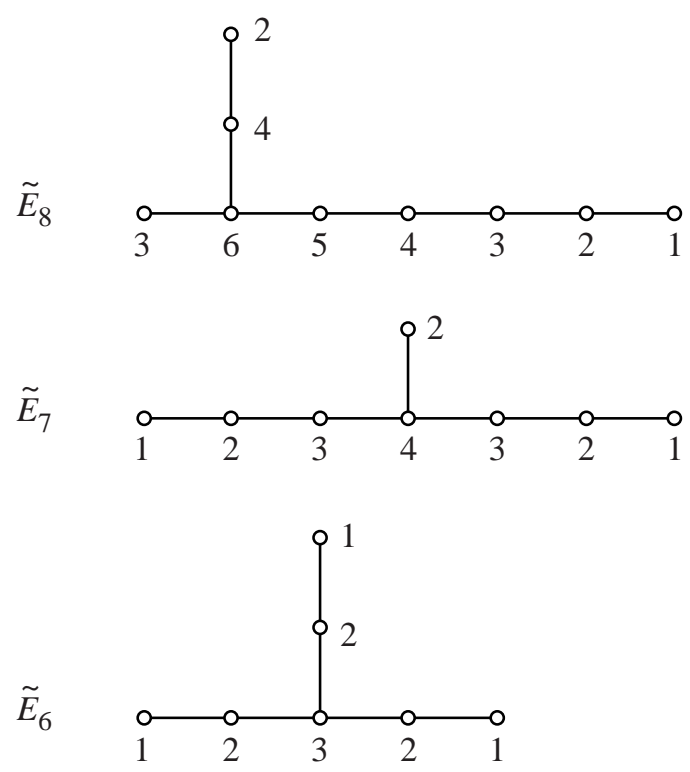

where in all cases the curve $C$ corresponds to the unique node of valence 3 and once again the fibration is elliptic. Otherwise $\widetilde{C}^{2}=-1$. We cannot however contract it to a canonical singularity of $(X, \mathcal{F})$ although we do observe that the fibre must arise as the intersection matrix of one of the following graphs, viz: 

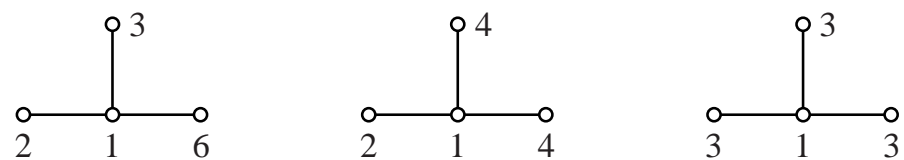

where now the weights denote the negative square of the self-intersection of the curve represented by the given node. In the first case contracting systematically and in order the curves with weights 1,2 and 3 yields a rational curve with a cusp, while in the second case contracting the curves of weight 1 and 2 yields $\widetilde{A}_{1}$, i.e. two rational curves tangent in one point, while in the final case contracting the curve of weight 1 yields 3 lines meeting in a point. Regardless in all cases we conclude that $\pi$ is an elliptic fibration.

So let us now take up the case of $k=4$ then we must have that $d_{1}=\cdots=$ $d_{4}=2$, whence $\widetilde{C}^{2}=-2$ and the fibre is necessarily the Dynkin diagram $\widetilde{D}_{4}$, and in particular forces $\pi$ to be an elliptic fibration.

Finally let us return to the cases $k=2, s_{\mathrm{I}}=3$ or $s_{0}=1$ then $\widetilde{C}^{2}=-1$ and the two -1-chains meeting $\widetilde{C}$ are in fact irreducible rational curves $E_{1}, E_{2}$ say of self-intersection -2 . Now if $s_{\mathrm{I}}=3$, then at say the intersection of $E_{1}$ and $C, E_{1}$ is the strong branch of a node which by I.3.1 would force $E_{1}^{2}=0$. Since this is nonsense we have $s_{0}=1$, and $s_{\mathrm{I}}=2$. Call the singularity not contained in $E_{1}$ or $E_{2}, z$. Again we can appeal to I.3.1 to see that the local contribution at $z$ to $C^{2}$ in the residue formula is zero, and this can only happen if $C$ is the strong branch of a node, i.e. in the Dynkin diagram notation we have,

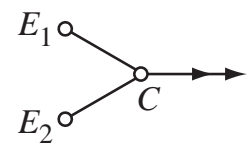

where the free edge corresponds to the weak branch of the node. $\square$

All of which is a very a good advertisement for stacks. Indeed by III.3.2 and IV.4.1, there is a smallest smooth stack, $p: \mathcal{X} \rightarrow X$ almost étale over the canonical model, and we let $f: \mathcal{C} \rightarrow \mathcal{X}$ be the normalisation of $p^{-1}(C)$. Appealing to the stack version of adjunction III.2.bis.1, we see that if $f$ does not meet the 
singular locus of the induced foliation then $\chi_{\mathcal{C}}=0$, and, needless to say $f$ is an isomorphism. On the other hand the moduli of $\mathcal{C}$ is rational, so the only possibilities are that it has 3 or 4 non-scheme like points. Should it be 4 then all the points have order 2 , i.e. $\widetilde{D}_{4}$, equivalently an elliptic curve modulo $\mathbb{Z} / 2$. Otherwise, the 3 non-scheme like points have orders one of $(2,3,6),(2,4,4)$, or $(3,3,3)$. The ambient dimension, however, is 2 , so there is only one possibility for a smooth stack with an isolated non-scheme like point of order 2 , and two possibilities for any of $3,4,6$. In particular the diagrams, $\widetilde{E}_{6}, \widetilde{E}_{7}, \widetilde{E}_{8}$, correspond to local group actions of the form,

$$
\mathbb{Z} / n \times \mathbb{A}^{2}: \zeta \times(x, y) \mapsto\left(\zeta x, \zeta^{-1} y\right)
$$

where $\zeta$ is a $n$th root of unity, for $n$ any of $2,3,4,6$, with the foliation given by $\frac{\partial}{\partial x}$, while the diagonal action corresponds to cases where the central node has weight 1 and square -1 in the minimal smooth resolution of $X$. Leaving aside the rational cases for the moment, we may also have the possibility that the image of $f$ has euler-characteristic zero, but fails to be smooth. Consequently there must be a node, which is either Gorenstein on $X$, i.e. $\widetilde{A}_{n}$, or non-Gorenstein (with perhaps a second node), i.e. $\widetilde{D}_{n}, n \geq 5$, cf. I.2.4 (e).

Manifestly, there is a corresponding discussion in terms of stacks for which $f(\mathcal{C})$ has negative Euler characteristic. A little thought, i.e. not just III.2.bis.1 but also I.2.4 (e), shows that this can only occur if $f$ is an isomorphism, and $\mathcal{C}$ meets the singular locus of the foliation in one or two points, with all such singularities being Gorenstein. In the latter case, these points could for an arbitrary stack be non-scheme like (albeit they must have the same stabiliser since the generic fibre is $\mathbb{P}^{1}$ ) but on the canonical model they are in fact scheme like, essentially because of how curves of square -1 were treated in III.3, cf. IV.1.2. In the former case, there must also be two non-scheme like points of order 2 . This is almost the case of the special rational curve in IV.4.5, and indeed is this case as soon as one knows that the singularity is scheme like. That it must be scheme like is because otherwise, the normal direction would force the stabiliser to be cyclic, while the non-Gorenstein terminal points imply that the group is $\mathbb{Z} / 2 \times \mathbb{Z} / n$, for $n$ either 2 or 4 , which is nonsense. Conversely,

Precision IV.4.6. The special rational curve at the end of IV.4.5 really does occur, and for $P=\mathbb{P}^{1} \times \hat{\Delta}, \hat{\Delta}$ a formal disc, it is the quotient of a foliation defined 
in suitable coordinates by,

$$
\partial=y \frac{\partial}{\partial y}+x^{2 p} \frac{\partial}{\partial x}
$$

under the action $x \mapsto-x, y \mapsto y^{-1}$, where $p \in \mathbb{N}$.

Proof Indeed a formal neighbourhood of the special curve of IV.4.5 certainly has a $\mathbb{Z} / 2$ covering by $P$, and the foliation is given by a global vector field on $P$, which goes to it's negative under the action. Some linear algebra, then gives the required coordinates, with $x$ the base and $y$ the fibre coordinates.

More generally, if the generic fibre of the Kodaira fibration $\pi: X \rightarrow B$ is $\mathbb{P}^{1}$, then there exists a generically scheme like proper one dimensional stack (which slightly abusively we'll call a curve) $\mathcal{B}$, such that the moduli of the fibration $\sigma: \mathcal{X} \rightarrow \mathcal{B}$ is just $\pi$, but now $\sigma$ is a conic bundle since every fibre $p^{-1}\left(X_{b}\right)$ has an étale covering by $\mathbb{P}^{1}$. Plainly no smooth fibre can be invariant by IV.1, while every fibre which contains a singularity must be invariant since in neighbourhoods of fibres the foliation is defined by a vector field. Consequently, the natural map, $\omega_{\mathcal{B}} \rightarrow K_{\mathcal{F}}$ vanishes exactly on the singular (equivalently invariant) fibres at $b_{1}, \cdots, b_{n}$, say, to some orders $p_{1}, \cdots, p_{n}$, so that,

$$
K_{\mathcal{F}}=\omega_{\mathcal{B}}\left(p_{1} b_{1}+\cdots p_{n} b_{n}\right)
$$

Outside of these fibres, and in the analytic topology, projecting along a leaf onto a fibre realises the foliation as a local system of $\mathbb{P}^{1}(\mathbb{C})$ 's, so that the foliation restricted to the complement of the singular fibres is analytically equivalent to a representation,

$$
r: \Gamma \rightarrow \mathrm{PGL}_{2}(\mathbb{C})
$$

where $\Gamma$ is the fundamental group of $\mathcal{B} \backslash\left\{b_{1}, \cdots, b_{n}\right\}$. The rather appealing nature of this description notwithstanding, one should be aware of,

Caveats IV.4.7. Apart from obvious stupidities that we should really talk about $\mathcal{B} \backslash\left\{b_{1}, \cdots, b_{n}\right\}$ rather than $\Gamma$, it is not true that $r$ determines the foliation. Indeed,

(a) The representation $r$ could very well be trivial. In fact even $\Gamma$ could be trivial, since there are no a priori conditions except,

$$
-\chi_{\mathcal{B}}+\left(p_{1} \operatorname{deg}\left(b_{1}\right)+\cdots+p_{n} \operatorname{deg}\left(b_{n}\right)\right)>0
$$


and canonical singularities.

(b) As such, there is an issue as to what additional data is required to obtain $(\mathcal{X}, \mathcal{F})$ given $r$. Plainly this takes the form of a compactification data about the singular fibres.

(c) Certainly this compactification data contains the $p_{i}$ 's, which together with $r$ determine (up to flipping the fibre) the formal isomorphism class of the completion in the singular fibre. This, however, is radically insufficient to determine the compactification data.

(d) Nor is the compactification data the analytic isomorphism class of an analytic neighbourhood of the singular fibre ( which incidentally has dimension $2 p_{i}-3$, for $p_{i} \geq 2$, cf. [MR]) but rather the analytic isomorphism class of the pair: an analytic neighbourhood of the singular fibre \& an analytic embedding into the smooth locus.

Let us, therefore, describe this compactification, around an analytic étale neighbourhood, $P=\mathbb{P}^{1} \times \Delta$, of a singular fibre at $p_{i}$, with $b_{i}$ identified to the centre of the unit disc $\Delta$. Plainly, giving $r$ specifies the analytic equivalence class of $\left(\left.P\right|_{\Delta^{\times}}, \mathcal{F}\right)$, and even that of $(P, \mathcal{F})$ if $p_{i}=1$, up to the unique possibility for non-uniqueness in the canonical model, i.e. flipping fibres. Indeed, up to the action of $\mathrm{PGL}_{2}$, we have two cases according to whether $\left.r\right|_{\pi_{1}\left(\Delta^{\times}\right)}$is semi-simple or not. In the later case the foliation is of the form,

Data IV.4.8.i $\partial=y\left(x^{n} y-n\right) \frac{\partial}{\partial y}+x \frac{\partial}{\partial x}$ for any non negative integer $n$, and, as will be the case throughout this discussion $(y, x)$ a standard coordinate system on $P$. In particular the singular fibre is wholly scheme like.

Similarly, in the semi-simple case, the foliation takes the form,

Data IV.4.8.ii $\partial=\lambda y \frac{\partial}{\partial y}+x \frac{\partial}{\partial x}$, for $\exp (2 \pi i \lambda)$ an eigenvalue of $r$ restricted to a loop around $b_{i}$, with $\lambda \notin \mathbb{Q}$, and again the fibre is wholly scheme like.

The indeterminacy in $\lambda$ with respect to $r$ corresponds exactly to the possibility of flipping fibres, while the canonical model procedure never allows the possibility that $p_{i}=1$, and $\lambda \in \mathbb{Q}$. As such, it's worth making,

Remark IV.4.9. There may well be loops in $\mathcal{B}$ around points other than the 
singularities where $r$ is non-trivial. With the exception of compactification data related to IV.4.6, these occur iff the fibre is non-scheme like. In particular since $\mathcal{X}$ is scheme like in co-dimension $1, r$ is necessarily faithful on such loops (whence $r$ is not quite arbitrary) so that if we have a non-scheme like fibre with local monodromy $n$ it is simply the foliation, $\partial=\frac{\partial}{\partial x}$ under the action, $x \mapsto \zeta x$, $y \mapsto \zeta^{a} y$, for $\zeta$ a $n$th root of unity, and $a$ relatively prime to $n$. Plainly such fibres cannot be flipped.

Certainly, therefore, any $r$ can be compactified over the singularities by an appropriate combination of IV.4.8.i/ii. This is not, however, the unique way to proceed, and there are a myriad of additional possibilities as soon as $p_{i}>1$. Now, we are interested in describing these possibilities on a fixed bi-rational model $X$, i.e. we exclude the possibility that two compactification data are isomorphic under a bi-rational automorphism of $X$. As such the compactification data for $p_{i}$ fixed is the analytic isomorphism class of foliations $\mathcal{F}$ on $P$, everywhere transverse to $P \rightarrow \Delta$ except over the origin, where the fibre is singular and invariant, under the action of the kernel of,

$$
\mathrm{PGL}_{2}(\Delta) \rightarrow \mathrm{PGL}_{2}(\mathbb{C}) \rightarrow 1
$$

In particular, we may, and do assume that the gluing of $\Delta$ to $\mathcal{B} \backslash\left\{b_{1}, \cdots, b_{n}\right\}$ implicit in IV.4.7 is by way of the standard embedding of $\Delta^{\times}$in $\Delta$. Consequently.

Data IV.4.8.iii. If the singular fibre is scheme like, then for $r$ fixed, the isomorphism classes of such data are isomorphic to a Zariski open of $\mathbb{A}^{3 p_{i}-3}$, and correspond to foliations defined by fields,

$$
\partial=x^{p_{i}} \frac{\partial}{\partial x}+\left(u(x) y^{2}+v(x) y+w(x)\right) \frac{\partial}{\partial y}
$$

where $u, v, w$ have degree at most $p_{i}-1$, and are determined in degree $p_{i}-1$ by $r$. While the Zariski open is $v^{2}(0) \neq 4 u(0) w(0)$.

Proof Given that $\Delta$ is fixed, not just up to isomorphism, we can certainly find a unique field, $\partial$, generating the foliation such that $\partial(x)=x^{p_{i}}$, and $\partial(y)$ as above, except that $u, v, w$ are analytic functions of $x$. At the same time, whatever the reduction of the data, i.e. $u, v, w$, under the group action may be, this data must patch uniquely to a foliation on $\mathbb{P}^{1} \times \mathbb{P}^{1}$ with a singular fibre of order $p_{i}$ over the origin, and a fibre over $\infty$ of order 1 (or exceptionally smooth if $r$ is trivial). 
Since bi-rational automorphism of $\mathbb{P}^{1} \times \mathbb{P}^{1}$ is excluded, we conclude by a simple explicit calculation.

We can combine this with IV.4.6 to complete our description of the compactification data, viz:

Data IV.4.8.iv. If the singular fibre is non-scheme like, then the compactification is the classifying stack of the $P$ with the foliation given by,

$$
\partial=x^{p_{i}} \frac{\partial}{\partial x}+\left(u(x) y^{2}+v\left(x^{2}\right) y+u(-x)\right) \frac{\partial}{\partial y}
$$

under the action $x \mapsto-x, y \mapsto y^{-1}$. Furthermore, $p_{i}=2 q_{i}$ is even, and $u$, $v$ have degrees at most $p_{i}-1$, and $q_{i}-1$ respectively. As such the moduli of the compactification data is isomorphic to the Zariski open, $v(0)^{2} \neq 4 u(0)^{2}$ in $\mathbb{A}^{\left(3 q_{i}-1\right)}$.

Before progressing, let us clarify the relation between the compactification data and IV.4.7 by way of,

Remark IV.4.9.bis. In IV.4.7. (c) we have stated that the isomorphism class of the completion in a singular fibre is uniquely determined by $r$, and $p_{i}$, as is indeed easily verified by linear algebra. An aspect of this is allowing formal isomorphisms of the disc. Nevertheless even if we restrict ourselves to the action of $\mathrm{PGL}_{2}$, the space of formal compactifications in IV.4.8.iii, given $r$, is only a $\mathbb{G}_{m} \times \mathbb{A}^{p_{i}-2}$, so there is a difference of $2 p_{i}-2$ dimensions, while in IV.4.8.iv, the difference is, $2 q_{i}-1$ dimensions. Now in IV.4.7. (d), we've said that the dimension up to local analytic isomorphism in IV.4.8.iii is $2 p_{i}-3$, but this includes the action of $\mathrm{PGL}_{2}(\mathbb{C})$, so the difference is $2 p_{i}-2$, on allowing germs of analytic isomorphism of the disc. These analytic isomorphism classes are precisely a result of the obstruction to the analytic convergence of the weak branch of the node, i.e. of an invariant $x$-axis, through either of the singularities on the invariant fibre, with the dimension of the obstruction at either singularity being $p_{i}-1$. Consequently, the compactification data in IV.4.8.iii is generically (i.e. there are some subtilties if $r$ is unipotent) the combination of the formal class with fixed isomorphism on $\Delta$ and the said obstructions. Similarly, due to the symmetries in IV.4.8.iv, the dimension of the obstruction to the convergence of one, whence either, weak branch is $2 q_{i}-1$, and the same conclusion holds. An explicit description of the 
local analytic structure, as opposed to the subterfuge of meromorphic extension in IV.4.8.iii, can be found in [MR].

The discussion on compactification completed, we may summarise all of this by way of noting it within the classification of foliations of Kodaira dimension 1, i.e.

Case IV.4.4(ii) (Riccatti foliations) If the generic fibre of the Kodaira fibration $\pi: X \rightarrow \widetilde{B}$ is a rational curve, then the foliation $(X, \mathcal{F})$ is the moduli of its Gorenstein covering stack $(\mathcal{X}, \mathcal{F})$, which in turn is defined by the following data,

(a) A finite map $p: \mathcal{B} \rightarrow B$ of generically scheme like smooth stacks.

(b) A possibly empty collection of closed points $b_{1}, \cdots, b_{n}$ of $\mathcal{B}$ which are either scheme like or of order 2 , with associated weights $p_{1}, \cdots, p_{n} \in \mathbb{N}$ which are arbitrary at scheme like points, even otherwise, and satisfy,

$$
p_{1} \operatorname{deg}\left(b_{1}\right)+\cdots+p_{n} \operatorname{deg}\left(b_{n}\right)>\chi_{\mathcal{B}}
$$

(c) A representation $r: \Gamma:=\pi_{1}\left(\mathcal{B} \backslash\left\{b_{1}, \cdots, b_{n}\right\}\right) \rightarrow \mathrm{PSL}_{2}(\mathbb{C})$ faithful on any elements of $\Gamma$ of finite order.

(d) A compactification data of the form IV.4.8.i-iv of the foliated classifying stack $\left[\mathbb{P}^{1} \times U \rightarrow \mathbb{P}^{1} / \Gamma\right]$, where $U$ is the universal (and necessarily scheme like by (c)) cover of $\mathcal{B} \backslash\left\{b_{1}, \cdots, b_{n}\right\}$, with $\Gamma$ acting diagonally by way of $r$. In particular the compactification $\mathcal{X} \rightarrow[\mathcal{X} / \mathcal{F}]$ exhibits $\mathcal{X}$ as a $\mathbb{P}^{1}$ bundle, $\pi: \mathcal{X} \rightarrow \mathcal{B}$ in the étale topus of $\mathcal{B}$ with moduli the Kodaira fibration.

If this description seems rather lengthy, it is because it is illustrative of the stacky nature of the theory, and the importance of the Gorenstein covering stack in particular. In fact, any invariant curve inside the canonical model of a foliation of general type will have for the specialisation of the foliation to it's normal cone, a Riccatti foliation with invariant hyperplane at infinity, so that the complexity of the description is a reflection of some of the complexity of foliations of general type. Furthermore, there is plainly very little difference between Riccatti foliations in the category of projective varieties, and the 2-category of algebraic stacks. Indeed the only differences that may occur are rather trivial, i.e. in (a) 
$\mathcal{B}$ need not be generically scheme like, in (b) the $b_{i}$ need not be scheme like, but the compactification data would remain the same up to a more general notion of flipping fibres and/or rendering the fibre less scheme like, which could also occur by dropping the unique condition in (c). As such, we learn that several things that one might hope to be true are actually false, e.g.

Corollaries IV.4.10. A rather different pattern of behaviour to standard Mori theory and/or foliated Kodaira dimension 0 emerges, i.e.

(a) The Gorenstein covering stack need not admit an étale covering by a projective surface.

(b) There is no integer $n$ such that $K_{\mathcal{F}}^{\otimes n}$ is a line bundle on $X$ for all Riccatti foliations $(X, \mathcal{F})$.

(c) On the plus side, however, there is a covering $h: S \rightarrow \mathcal{X}$ of the Gorenstein covering stack by a smooth algebraic surface with at worst ramification along an invariant fibre. As such the induced canonical bundle is $h^{*} K_{\mathcal{F}}$, i.e. $h$ is étale in the foliation direction.

Proof Suppose (a) were false, and $S \rightarrow \mathcal{X}$ was an étale cover, then the Kodaira fibration $S \rightarrow C$ would give an étale cover of $\mathcal{B}$ by an honest curve, which is nonsense since $\mathcal{B}$ is arbitrary. In fact if we take for $\mathcal{B}$ the simply connected stack whose moduli is $\mathbb{P}^{1}$, with a non-scheme like point of order $m$ over the origin and glue it to a singular fibre at infinity with $p=2$, then, as a bundle on $\mathcal{B}, K_{\mathcal{F}}$ has degree, $1-\frac{1}{m}$, which also proves (b). Finally (c) holds because $\mathcal{B} \backslash\left\{b_{1}, \cdots, b_{n}\right\}$ has finite étale covers by honest curves.

Should, however, the generic fibre of the Kodaira fibration be elliptic, then the corresponding discussion is decidedly more straightforward. Again, outside of any singular and or invariant fibres, the foliation is given by a representation in the automorphism group of an elliptic curve. As such the conformal structure on the fibres cannot degenerate, so that no fibre is a canonical model of an $\widetilde{A}_{n}, n \geq 2$, a $\widetilde{D}_{n}, n \geq 5$, or indeed a rational curve with a cusp. As such the tabulation of IV.4.5 not only reveals that the Gorenstein covering stack $p: \mathcal{X} \rightarrow X$ is smooth, but also that the induced foliation $(\mathcal{X}, \mathcal{F})$ is a smooth foliation. Better still to the Kodaira fibration $\pi: X \rightarrow B$, there corresponds a smooth map of smooth 
stacks, $\pi: \mathcal{X} \rightarrow \mathcal{B}$ with the same moduli. Since, however, fibres of the Kodaira fibration may now be multiple even if $X$ is smooth, it follows that $\mathcal{X} \rightarrow \mathcal{B}$ differs from $X \rightarrow B$ both at fibres through singularities of $X$, and multiple fibres. As such, $\pi: \mathcal{X} \rightarrow \mathcal{B}$ is an elliptic fibres bundle with typical fibre $E$, and we have the exact sequence,

$$
1 \longrightarrow E \longrightarrow \operatorname{Aut}(E) \longrightarrow \mu_{n} \longrightarrow 1
$$

where $n$ is 2,4, or 6 (in fact 2 unless $E$ is the curve with automorphic complex multiplication by $\mu_{4}$, respectively $\mu_{6}$ ). In particular, any finite cyclic sub-group is either contained in $E$, or disjoint from $E$, so there is a finite étale cover $q: \mathcal{C} \rightarrow \mathcal{B}$ whose fibres over non-scheme like points are either: wholly non-scheme like if the non-scheme like point on $\mathcal{B}$ arises from a multiple fibre, wholly scheme like if it arose from a singularity of $X$. Consequently, the étale cover,

$$
S:=\mathcal{X} \times{ }_{\mathcal{B}} \mathcal{C}
$$

is a scheme, or, if one prefers: the canonical model $(X, \mathcal{F})$ admits an almost étale cover, $(S, \mathcal{F}) \rightarrow(X, \mathcal{F})$, by a smoothly foliated smooth surface, and the induced Kodaira fibration, $\pi: S \rightarrow C$ has no fibres whose reduction is singular. Better still the fact that the foliation $\mathcal{F}$ on $S$ is smooth, forces $K_{\mathcal{F}}$ to be a bundle on $C$, so all multiple fibres are invariant.

This suggests that we move the phenomenon of multiple fibres to the compactification data, since they are contained in the set $\left\{c_{1}, \cdots, c_{n}\right\}$ of invariant fibres, and since $K_{\mathcal{F}}$ is a bundle on $C$, the map $\omega_{C} \rightarrow K_{\mathcal{F}}$ vanishes along $S_{c_{i}}$ to some integral multiple $p_{i}$ of $S_{c_{i}}$, so that the condition for Kodaira dimension 1 reads,

$$
p_{1}+\cdots p_{n}>\chi_{C}
$$

While, as before we have a representation,

$$
r: \Gamma:=\pi_{1}\left(C \backslash\left\{c_{1}, \cdots, c_{n}\right\}\right) \longrightarrow E
$$

To determine the compactification data, at least for non-multiple fibres, we can have recourse to IV.4.8 on viewing $E$ as a quotient of $\mathbb{G}_{m}$, so none of the subtleties of IV.4.9 occur, i.e. formal and analytic data coincide, whence for fixed $r$, the data is a $\mathbb{G}_{m} \times \mathbb{A}^{p_{i}-2}$ (trivial if $p_{i}=1$ ). In the multiple case, we can view it as a quotient of this case by $\mathbb{Z} / n$ for a suitable $n$, and on computing the 
consequences of the various constraints find that the space of compactification data is unchanged, which we may as well note by way of,

Data IV.4.11. The isomorphism class of the foliation around a punctured neighbourhood of an invariant fibre of $S$ has for each $n \in \mathbb{N}$ a compactification by a fibre of order $n$, and the space of such compactifications is isomorphic to a $\mathbb{G}_{m} \times \mathbb{A}^{p_{i}-2}$ (trivial if $p_{i}=1$ ).

All of which may be summarised via its place in the classification, i.e.

Case IV.4.4(iii) (Turbulent foliations) If the generic fibre of the Kodaira fibration $\pi: X \rightarrow B$ is an elliptic curve then $(X, \mathcal{F})$ has an almost étale Kummer covering, $(S, \mathcal{F}) \rightarrow(X, \mathcal{F})$ by a smoothly foliated smooth surface arising from a Kummer covering $C \rightarrow B$ such that the induced foliation is described by,

(a) A possibly empty collection of points $c_{1}, \cdots, c_{n}$ of $C$, with associated weights $p_{1}, \cdots, p_{n}$ satisfying,

$$
p_{1}+\cdots p_{n}>\chi_{C}
$$

(b) A representation $r: \Gamma=\pi_{1}\left(C \backslash\left\{c_{1}, \cdots, c_{n}\right\}\right) \rightarrow E$, where $E$ is an elliptic curve.

(c) A compactification data of the form IV.4.11 of the foliated classifying stack $[E \times U \rightarrow E / \Gamma]$, where $U$ is the universal cover of $C \backslash\left\{c_{1}, \cdots, c_{n}\right\}$.

Better still the Kummer covering is the quotient of this data by a group of automorphisms isomorphic to $\mathbb{Z} / n$, for $n$ one of $1,2,3,4,6$, all of which may occur, albeit 4 requires $j$ invariant 1728 , and 3 or $6, j$ invariant 0 .

In particular, none of the complication of IV.4.10 occurs, and as one might expect things just keep getting easier as the genus of the generic fibre of the Kodaira fibration increases. Indeed by IV.4.5, should the genus be at least 2 then the canonical model $X$ is smooth, as is the foliation, nor does the Kodaira fibration have multiple fibres since a hyperbolic curve cannot be invariant and have degree 0 along $K_{\mathcal{F}}$. Consequently we may note,

Case IV.4.4(iv) (Final Case) If the generic fibre of the Kodaira fibration $\pi$ : $X \rightarrow B$ is hyperbolic, then $(X, \mathcal{F})$ is a smooth foliation on a smooth surface everywhere transverse to $\pi$. As such $K_{\mathcal{F}}=\pi^{*} \omega_{B}$, so $B$ is hyperbolic, and after 
a finite étale cover $\widetilde{B} \rightarrow B$, the induced foliation is a product $C \times \widetilde{B} \rightarrow C$, with fibres $\widetilde{B}$, for some higher genus curve $C$. Other foliations which are isotrivial families of genus at least 2 over non-hyperbolic basis have already been implicitly noted previously, and occur for empty compactification data of finite representations.

It should, therefore, be clear, that the natural language for cases IV.4.4.ii-iv, and to some extent even IV.4.4.i is algebraic stacks. Essentially the full gambit of additional complication that might occur on studying canonical models of arbitrary stacks is not substantially greater than IV.4.4.ii. Plainly, the basic strategy of proceeding via the structure of the generic fibre changes little, with the only real issue being that there are countably many simply connected 1dimensional parabolic stacks, albeit that these can clearly be treated as Riccatti foliations with extra structure. Indeed this extra structure is often simplifying, as in the quasi-projective case where there is nothing to add to or list except for the generic fibre being $\mathbb{A}^{1}$ or $\mathbb{G}_{m}$, which are just Riccatti foliations with half, respectively all, of the complication of IV.4.9 removed.

\section{IV.5. Sporadic foliations}

We begin with a lemma which shows that we have a stark choice i.e. if $\nu(X, \mathcal{F})=1$, where as ever $(X, \mathcal{F})$ is the canonical model of a foliated surface then either $\kappa(X, \mathcal{F})=1$ or $\kappa(X, \mathcal{F})=-\infty$. Specifically we prove,

Proposition IV.5.1. Hypothesis as above if $\kappa(X, \mathcal{F}) \geq 0$ then in fact $\kappa(X, \mathcal{F})=$ 1 , so that in particular the classification is as per $I V .4 .4$

Proof. Suppose indeed $\kappa(X, \mathcal{F}) \geqq 0$, then there is an effective $\mathbb{Q}$-Cartier divisor, $\sum a_{i} C_{i}$, say, where $C_{i}$ are irreducible curves which represents $K_{\mathcal{F}}$, and by IV.4.1 $(X, \mathcal{F})$ is $\mathbb{Q}$-foliated Gorenstein, and $C_{i}^{2}=C_{i} \cdot C_{j}=0 \forall i, j$. Now we consider various cases.

Firstly suppose some curve $C$ from amongst the $C_{i}$ is an integral sub-curve of $\mathcal{F}$. Then either $C$ meets the singular locus of $X$ or it does not. Should $C$ not meet the locus where the underlying space $X$ is singular then either $C$ is an elliptic curve or a rational curve with a node. In either case, $K_{X} \cdot C=C^{2}=0$ and $C$ is a divisor of elliptic fibre type. Alternatively $C$ meets $\operatorname{sing}(X)$, and we 
may consider the unique minimal smooth resolution $\rho: \widetilde{X} \rightarrow X$. The argument of IV.4.5 goes through verbatim to establish that $\rho^{*} C$ is again of elliptic fibre type, or the special rational fibre at the end of the proof. However this latter certainly moves in a 1-dimensional family. However if $q(X)>0$ then the rational fibration determined by $C$ is the Albanese, so certainly in this case $k(\mathcal{F})=1$. Now it is well known that a divisor of elliptic fibre type on a surface of positive, $K_{X}$, Kodaira dimension must arise from an elliptic fibration, and so in this case we may conclude. However, cf. $[R]$, this is probably the least attractive part of ordinary classification, and in addition is not true for rational surfaces. Whence we will prove:

Divertimento IV.5.1 (bis) Let $D$ on $\widetilde{X}$ be a divisor of elliptic fibre type such that $D_{\text {red }}$ is invariant by $\widetilde{\mathcal{F}}$ then $K_{\mathcal{F}}$ has Kodaira dimension 1 , and the $K_{\mathcal{F}}$ Kodaira fibration is an elliptic fibration.

Proof. By IV.4.5 it suffices to show that the foliation has Kodaira dimension 1. We consider cases according to the irregularity. Observe that if the foliation is a fibration, then by Zariski's lemma it is an elliptic fibration and so we need only consider, $q(\widetilde{X})=2$ and $h^{0}\left(K_{\widetilde{\mathcal{F}}}\right)=1, q(\widetilde{X})=1$ and $h^{0}\left(K_{\tilde{\mathcal{F}}}\right)=1$, or $q(\widetilde{X})=0$. In either of the cases $q(\widetilde{X})>0$ the divisor $D$ must be a genuine elliptic curve, and the Albanese map must be to either an abelian surface or an elliptic curve. Consider the case $q(\widetilde{X})=1$, then we can suppose without loss of generality that $D$ is in the support of the map from $\omega_{\operatorname{Alb}(\widetilde{X})} \rightarrow K_{\mathcal{F}}$. Indeed, we have a commutative diagram of natural maps,

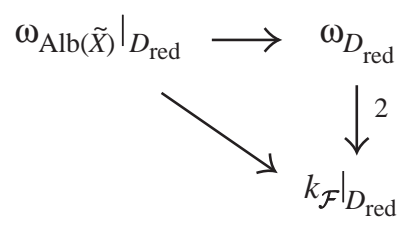

so the composite diagonal map is zero, and the Albanese fibration is the desired fibration. Should $q(\widetilde{X})=2$, we may reduce to the same situation after taking étale covers, on replacing the Albanese map by the map from $\operatorname{Alb}(\widetilde{X})$ to the image of $D$ in the same obtained via Poincaré decomposition.

Thus by Riemann-Roch, we are reduced to studying the case where $q(\widetilde{X})=$ 
$p_{g}(\tilde{X})=0$. Call the image of $D_{\text {red }}$ in $(X, \mathcal{F}), C$, then for some $m, n \in \mathbb{N}$ we must have, $m K_{\mathcal{F}}=n C$. Suppose that $m, n$ have a common factor $l$, say, then $m=l m_{0}, n=l n_{0}$ with $m_{0}, n_{0} \in \mathbb{N}$ and, $l\left(m_{0} K_{\mathcal{F}}-n_{0} C\right)=0$. So we may pass to a cover which is étale in codimension 1, and apply IV.3.3 to conclude without loss of generality $(m, n)=1$. Now consider the cover in $\mathbb{V}\left(K_{\mathcal{F}}^{-m}\right)$ defined in codimension 2, corresponding to a $m^{\text {th }}$-root of $n C$, and define a scheme $Y$ by taking $\mathcal{O}_{Y}$ to be the normalisation of $\mathcal{O}_{X}$ in the function field of the same. This gives us a finite cover $\nu: Y \rightarrow X$ ramified over $C$, which locally in codimension 2 just corresponds to taking a $m^{\text {th }}$-root of a local equation for $C$. Whence there is a Weil divisor $C^{\prime}$ on $Y$ such that, $\nu^{*} C=m C^{\prime}$ as Weil divisors. Better still since $C$ is an integrable sub-curve on $X, K_{\mathcal{G}}=\nu^{*} K_{\mathcal{F}}$, again a priori as Weil divisors, but a postiori as $\mathbb{Q}$-Cartier divisors since $K_{\mathcal{F}}$ is $\mathbb{Q}$-Cartier on $X$. The proof of IV.3.3 only required that this identity be satisfied, so $(Y, \mathcal{G})$ has canonical singularities. Whence modulo passing to yet another cover, this time étale in codimension 1 to kill any torsion, and appealing to the canonical model theorem in its full generality for singular spaces we may assume without loss of generality that, $K_{\mathcal{F}}=n C$.

We now distinguish various cases according to the type of $C$,

(a) $C$ is an elliptic curve. By adjunction, as applied in IV.4.5, $C$ does not meet $\operatorname{sing}(X)$ and so $(X, \mathcal{F})$ is foliated Gorenstein. Whence by III.1.4, $K_{\widetilde{\mathcal{F}}}=\rho^{*} K_{\mathcal{F}}$, and Riemann-Roch implies $H^{1}\left(\widetilde{X}, K_{\widetilde{\mathcal{F}}}\right)=0$. However $C$ is contained in the smooth locus of the foliation so we conclude to the absurdity that,

$$
c_{1}(C) \in \operatorname{Im}\left\{H^{1}\left(\widetilde{X}, K_{\tilde{\mathcal{F}}}\right)^{\vee} \rightarrow H^{1,1}(\widetilde{X})\right\}
$$

(b) $\rho^{*} C$ is a rational curve with a node or an elliptic polygon. This is similar to (a). Again $(X, \mathcal{F})$ is foliated Gorenstein, and $K_{\widetilde{\mathcal{F}}}=\rho^{*} K_{\mathcal{F}}=n D$, where $D$ is the said elliptic divisor, so that once more Riemann-Roch gives $H^{1}\left(\widetilde{X}, K_{\widetilde{\mathcal{F}}}\right)=0$. Now in general an elliptic polygon is not in,

$$
\operatorname{Im}\left\{H^{1}\left(\widetilde{X}, K_{\tilde{\mathcal{F}}}\right)^{\vee} \rightarrow H^{1,1}\right\}
$$

However in this particular case $D$ is properly the Dynkin diagram $\widetilde{A}_{n}$, i.e. all components have self intersection -2 , and an explicit calculation using I.3.1 shows that this can only happen if the eigenvalue at each singularity is -1 . Consequently 
the obstruction posed by the residue map is zero, so we once more have the absurdity that $D$ is zero in $H^{1,1}$.

(c) The elliptic divisor $D$ corresponding to the appropriate multiple of $\rho^{*} C$ is one of the Dynkin diagrams $\widetilde{D}_{n}, n \geq 4, \widetilde{E}_{6}, \widetilde{E}_{7}, \widetilde{E}_{8}$.

Since every component of $D$ has self intersection -2 and $(X, \mathcal{F})$ is Gorenstein off $D$ we have $K_{\widetilde{\mathcal{F}}}-D_{\text {red }}=\rho^{*}\left(K_{\mathcal{F}}-C\right)$, and $D_{\text {red }}^{2}=-2$. Consequently RiemannRoch gives $\chi\left(\widetilde{X}, K_{\widetilde{\mathcal{F}}}\right)=0$, and whence, $h^{1}\left(\widetilde{X}, K_{\widetilde{\mathcal{F}}}\right)=1$. As ever consider the dévissage defining the foliation, i.e.

$$
0 \rightarrow N \rightarrow \Omega_{\widetilde{X}}^{1} \rightarrow K_{\widetilde{\mathcal{F}}} \cdot I_{Z} \rightarrow 0
$$

for $Z$ the singular subscheme of $\widetilde{\mathcal{F}}$, then we get an exact sequence,

$$
0 \rightarrow H^{0}\left(\widetilde{X}, K_{\widetilde{\mathcal{F}}} \cdot I_{Z}\right) \rightarrow H^{1}(\widetilde{X}, N) \rightarrow H^{1,1}
$$

Now $D$ is certainly in the image of the last map whether by explicit calculation, or the fact that the number of curves exceeds the number of singularities. However $h^{1}(\widetilde{X}, N)=1$, so there must be foliation singularities, say $Z^{\prime}$, other than those supported in $D$. The next thing we observe is that for $m \gg 0, H^{0}\left(\widetilde{X}, K_{\widetilde{\mathcal{F}}}+\right.$ $\left.D_{\text {red }}(-m D)\right)=0$, so that Riemann-Roch gives $H^{0}\left(\widetilde{X}, N\left(-D_{\text {red }}+m D\right)\right) \neq 0$. In particular for some effective divisor $E$, possibly zero, supported off $D, m_{i} \in \mathbb{Z}$, and $C_{i}$ curves supported in $D, N=E+\sum m_{i} C_{i}$. However $\rho^{*} K_{\mathcal{F}} \cdot N=0=\rho^{*} K_{\mathcal{F}} \cdot C_{i}$, so $\rho^{*} K_{\mathcal{F}} \cdot E=0$, and $E$ must be supported on $-2 F$ curves. Denoting by $X^{\prime}$ the contraction of the $-2 F$ curves off $D$, we therefore have that $N\left(-\sum m_{i} C_{i}\right)$ is a torsion $\mathbb{Q}$-Cartier divisor on $X^{\prime}$, so modulo an almost étale cover we can suppose that $N\left(-\sum m_{i} C_{i}\right)$ is Cartier, which obliges any $-2 F$ curve to have self intersection -2 , and $E$ to be zero. Consequently without loss of generality we have a global meromorphic 1 form $\omega$ in $N$ with poles at most on $D$, so naturally $d \omega$ is a 2-form with poles of the said form and the issue is whether it's zero or not. Now there are definitely foliation singularities off $D$ on $\widetilde{X}$, and by Zariski's lemma $N$ is a negative multiple of $D$, and $D$ comes from $H^{1}(X, N)$, so the residue of $N$ at every singularity off $D$ is zero. However, quite generally, the image of $c_{1}(N)$ under the residue map is to $1^{\text {st }}$ order the trace of the linearisation of the vector field at the singularity, so all the eigenvalues off $D$ must be -1 , whence if $d \omega \neq 0$ there is a non-zero effective Cartier divisor $E$ supported off $D$, and 
$m_{i} \in \mathbb{Z}$ with as before $C_{i}$ curves supported in $D$ such that, $K_{X}=E+\sum m_{i} C_{i}$ and again $K_{\mathcal{F}} \cdot K_{X}=K_{\mathcal{F}} \cdot C_{i}$ forces $E$ to be supported on $-2 F$ curves, so once more modulo an almost étale cover $E=0$, which is absurd unless the cover destroys the foliation singularities off $D$, which we might as well assume doesn't happen, so without loss of generality $\omega$ is closed. Given that $\omega$ has meromorphic poles, it's class in $H_{D R}^{1}(\widetilde{X} \backslash D)$ is non-zero, unless we have an elliptic fibration in which case we'd be done, so without loss of generality $(\widetilde{X}, D)$ has non-trivial logarithmic Albanese. However here we have a dévissage of the form,

$$
0 \rightarrow N\left(D_{\text {red }}\right) \rightarrow \Omega^{1}\left(\log D_{\text {red }}\right) \rightarrow K_{\mathcal{F}} \cdot I^{\prime} \rightarrow 0
$$

where $I^{\prime}$ is a non trivial ideal with $\mathcal{O}_{\widetilde{X}} / I^{\prime}$ supported off $D$, so in fact $h^{0}\left(\widetilde{X}, K_{\mathcal{F}}\right.$ $\left.I^{\prime}\right)=0$, and by the closure of global forms with logarithmic poles, the foliation must again be an elliptic fibration.

(d) Otherwise. There are 3 remaining possibilities for the elliptic divisor $D$ by IV.4.5. In all of these $\widetilde{C}$ is an exceptional curve of the $1^{\text {st }}$ kind, $K_{\widetilde{\mathcal{F}}}=\rho^{*}\left(K_{\mathcal{F}}+\right.$ $C)-\widetilde{C}$ so again $\chi\left(\widetilde{X}, K_{\widetilde{\mathcal{F}}}\right)=0$, whence (c) goes through verbatim.

Consequently no curve $C$ from amongst the $C_{i}$ is an integral sub-curve, and indeed for any $C$ in the support of $K_{\mathcal{F}}$ with $\rho: \widetilde{X} \rightarrow X$ as above, and $\widetilde{C}$ its proper transform we may appeal to the proof of II.4.1 and III.1.1 to conclude that in fact, $\left(K_{\widetilde{\mathcal{F}}}+\widetilde{C}\right) \cdot \widetilde{C}=\left(K_{\mathcal{F}}+C\right) \cdot C=0$. Better still,

(a) $\widetilde{\mathcal{F}}$ is smooth in a neighbourhood of $\widetilde{C}$.

(b) $\mathcal{O}_{\widetilde{C}}(-\widetilde{C})$ embeds as a line bundle in $\Omega_{\widetilde{X}}^{1} \otimes \mathcal{O}_{\widetilde{C}}$, so in fact $\widetilde{C}$ is smooth.

(c) $K_{\mathcal{F}}(C)$ is Cartier, equivalent to a $\mathbb{Q}_{+}$multiple of the $\mathbb{Q}$-Cartier divisor $C$.

In particular $(X, \mathcal{F})$ is foliated Gorenstein iff $C$ is Cartier, which is in fact iff $\widetilde{X}$ is isomorphic to $X$ around $C$, but all we'll really use is that the minimum integer $n$ such that $\mathcal{O}_{X}\left(n K_{\mathcal{F}}\right)$ or $\mathcal{O}_{X}(n C)$ is Cartier is the same for $K_{\mathcal{F}}$ and $C$. In any case $T_{\mathcal{F}}(-C)$ is also Cartier, so for a suitable open cover indexed by $\alpha \in A$, let $\partial_{\alpha}$ be a local generator for $T_{\mathcal{F}}(-C)$ and $x_{\alpha}$ the same for $\mathcal{O}_{X}(-n C)$. Call the respective transition functions $g_{\alpha \beta}$ and $f_{\alpha \beta}$ then,

$$
\frac{\partial_{\alpha} x_{\alpha}}{x_{\alpha}}=\frac{\partial_{\alpha} f_{\alpha \beta}}{f_{\alpha \beta}}+\frac{\partial_{\alpha} x_{\beta}}{x_{\beta}}=g_{\alpha \beta} \frac{\partial_{\beta} x_{\beta}}{x_{\beta}} \quad\left(\bmod \mathcal{I}_{C}\right)
$$

and so $\partial_{\alpha} \log x_{\alpha}$ gives a global section of $\mathcal{O}_{C}\left(K_{\mathcal{F}}+C\right)$, which outside of the 
singularities is just $n$-times the usual section given by adjunction. Consequently it's non-zero, and gives an isomorphism $\mathcal{O}_{C} \stackrel{\sim}{\longrightarrow} \mathcal{O}_{C}\left(K_{\mathcal{F}}+C\right)$ since $\left(K_{\mathcal{F}}+C\right) \cdot C=$ 0 forces it to be nowhere vanishing. Now for $k \in \mathbb{N}$, let $X_{k}$ be the thickening of $C$ in $X$ by the $k^{\text {th }}$ symbolic power $\mathcal{I}_{C}^{(k)}=\mathcal{O}_{C}(-k C)$, and let's set about proving by induction that $\partial_{\alpha} \log x_{\alpha}$ also gives an isomorphism $\mathcal{O}_{X_{k}} \stackrel{\sim}{\longrightarrow} \mathcal{O}_{X_{k}}\left(K_{\mathcal{F}}+C\right)$. Quite generally we have exponential type sequences,

$$
\begin{gathered}
0 \longrightarrow \mathcal{I}_{C}^{(k)} / \mathcal{I}_{C}^{(k+1)} \stackrel{\exp =1+x}{\longrightarrow} \mathcal{O}_{X_{k+1}}^{*} \longrightarrow \mathcal{O}_{X_{k}}^{*} \longrightarrow 0 \\
H^{0}\left(\mathcal{O}_{X_{k}}^{*}\right) \stackrel{\delta}{\longrightarrow} H^{1}\left(C, \mathcal{I}_{C}^{(k)} / \mathcal{I}_{C}^{(k+1)}\right) \longrightarrow \operatorname{Pic}\left(X_{k+1}\right) \longrightarrow \operatorname{Pic}\left(X_{k}\right) \longrightarrow 0 .
\end{gathered}
$$

where the latter is deduced from the former. The basic fact to note is,

Fact. $\operatorname{Im}(\delta)$ is a sub complex vector space of $H^{1}\left(C, \mathcal{I}_{C}^{(k)} / \mathcal{I}_{C}^{(k+1)}\right)$.

Indeed for any $\rho \in \mathbb{C}$, and $h \in \mathcal{O}_{X_{k+1}}$ congruent to 1 modulo $\mathcal{I}_{C}$, we can define $h^{\rho}$ by the usual Taylor expansion, so that indeed if $g$ is another such function, $(h g)^{\rho}=h^{\rho} g^{\rho}$. On the other hand if $h \in H^{0}\left(\mathcal{O}_{X_{k}}^{*}\right)$ then its restriction to $C$ is a global unit, so a constant, say $\lambda \in \mathbb{C}^{\times}$and $\delta(h)=\delta(h / \lambda)+\delta(\lambda)=\delta(h / \lambda)$, so to calculate $\delta(h)$ we can suppose without loss of generality that it's congruent to 1 modulo $C$. Lifting $h$ with respect to our cover to $\mathcal{O}_{X_{k+1}}^{*}$ gives $p_{\alpha} \in \mathcal{I}_{C}$ with $h=\left(1+p_{\alpha}\right)$, and $\left(1+p_{\alpha}\right)\left(1+p_{\beta}\right)^{-1}=1+p_{\alpha \beta}$, for $p_{\alpha \beta} \in \mathcal{I}_{C}^{(k)} / \mathcal{I}_{C}^{(k+1)}$ a 1 cocycle representing $\delta(h)$. However $\left(1+p_{\alpha}\right)^{\rho}\left(1+p_{\beta}\right)^{-\rho}=\left(1+p_{\alpha \beta}\right)^{\rho}=1+\rho p_{\alpha \beta}$, so $\delta\left(h^{\rho}\right)=\rho \delta(h)$, and we're done.

Now let's offer the precision that our cover is in the étale topology. For some positive integers $p$ and $q$ we have that $p\left(K_{\mathcal{F}}+C\right)=q(n C)$ on $X$, so our induction hypothesis gives that for a $q$-torsion valued cocycle $\zeta_{\alpha \beta}$ and a 1-cocycle $h_{\alpha \beta} \in \mathcal{I}_{C}^{(k)} / \mathcal{I}_{C}^{(k+1)}$ we have, $f_{\alpha \beta}=\zeta_{\alpha \beta}\left(1+h_{\alpha \beta}\right)$. Equally for some function $u_{\alpha \beta}$, $h_{\alpha \beta}^{n}=u_{\alpha \beta} x_{\beta}^{k}$ in $\mathcal{O}_{X}$, so that $\frac{\partial_{\alpha} x_{\alpha}}{x_{\alpha}}=\exp \left(\frac{k}{n} h_{\alpha \beta}\right) g_{\alpha \beta} \frac{\partial_{\beta} x_{\beta}}{x_{\beta}}$. Consequently if $L$ is the bundle with transition functions $\exp \left(-\frac{h_{\alpha \beta}}{n}\right)$ then, $\partial_{\alpha} \log x_{\alpha}$, gives an isomorphism $\mathcal{O}_{X_{k+1}} \stackrel{\sim}{\longrightarrow} \mathcal{O}_{X_{k+1}}\left(K_{\mathcal{F}}+C\right) \otimes L^{k}$. From which we obtain, $L^{k p} \stackrel{\sim}{\longrightarrow}$ $\mathcal{O}(-q n C) \stackrel{\sim}{\longrightarrow} L^{-n q}$, so: $(n q+k p) L=0$, and by our vector space fact we've finished our induction. Better still since $C$ is $\mathbb{Q}$-Cartier the symbolic powers are certainly co-final in the $\mathcal{I}_{C}$-adelic topology, so if $\widehat{X}$ is the completion of $X$ in $C$ then $\operatorname{Pic}(\widehat{X})=\lim \operatorname{Pic}\left(X_{k}\right)$ and so in fact we've established that the Hilbert scheme of $X$ has positive dimension in a neighbourhood of $q n C$ (just consider 
the family $\mathbb{1}_{n C}^{q}=$ const). Whence there is a curve $A$, a family of curves $\nu: Y \rightarrow A$ with $n C$ as a fibre, embedding in $X \times A$, such that the projection $\mu: Y \rightarrow X$ is dominant, and $\mu^{*} K_{\mathcal{F}}$ is an effective $\mathbb{Q}$-Cartier divisor whose intersection with the generic fibre of $A$ is zero. Whence if $Y^{\#}$ and $A^{\#}$ are the respective normalisations of $Y$ and $A$ then $\left.K_{\mathcal{F}}\right|_{Y \#}$ is the pull-back of a $\mathbb{Q}$-Cartier divisor on $A^{\#}$ so that $K_{\mathcal{F}}$ has Kodaira dimension 1 as required.

Next keeping to the hypothesis $\nu(X, \mathcal{F})=1$, we do the irregular case, i.e.

Lemma IV.5.2. If $(X, \mathcal{F})$ is the canonical model of a foliated surface which is not a fibration by rational curves and $\rho:(\widetilde{X}, \widetilde{\mathcal{F}}) \rightarrow(X, \mathcal{F})$ the unique smooth resolution, such that the underlying space $\widetilde{X}$ has $q(\widetilde{X}) \geq 1$, then $\kappa(X, \mathcal{F}) \geq 0$. Nor can the foliation be a fibration if $\kappa(X, \mathcal{F})=-\infty$.

Proof. We recall as in IV.3.1 that by restricting global holomorphic 1 -forms to $K_{\tilde{\mathcal{F}}}$ we see that the lemma is trivial, unless $h^{0}(\widetilde{X}, N) \geq 2$ if $q(\widetilde{X}) \geq 2$ or $h^{0}(X, N)=1$ if $q(\tilde{X})=1$, where as ever $0 \rightarrow N \rightarrow \Omega_{\tilde{X}}^{1}$ is the saturated map defining the foliation. In the first case a well-known lemma of Castelnuovo, cf. [B1], implies the foliation is a fibration while in the second case it must be the Albanese fibration. In either case the quotient space $\widetilde{X} / \widetilde{\mathcal{F}}$ exists by virtue of the singularities being canonical, and better still the quotient map $\pi: \widetilde{X} \rightarrow B:=\widetilde{X} / \widetilde{\mathcal{F}}$ admits a factorisation through $\pi: X \rightarrow B$ where $B$ is equally $X / \mathcal{F}$ and of course $B$ is a smooth curve.

Now if $\pi$ is an elliptic fibration then we may conclude by IV.3.1 and IV.4, otherwise the generic fibre has genus $g \geq 2$, and we're basically in the situation of a theorem of Arakelov, cf. [S2], which even works, and in fact with a simpler proof, in positive characteristic, albeit that in op.cit. the fibration $X \rightarrow B$ is assumed to be semi-stable. This is, however, a limited problem. Indeed consider a semi-stable reduction of the fibration $\pi$, viz: 


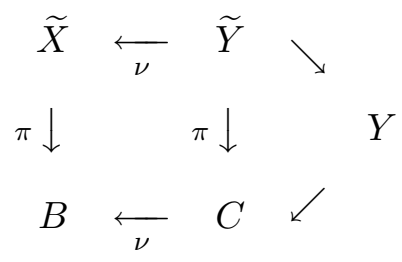

with induced foliation/fibration $\widetilde{\mathcal{G}}, \mathcal{G}$ on $\tilde{Y}$ and $Y$ respectively. The map $\nu$ is ramified only in fibres and is chosen in such a way that blowing down exceptional curves of the $1^{\text {st }}$ kind in the fibres leads to a non-singular $Y$ fibring over $C$ with semi-stable fibres. From the foliation point of view $Y$ is the minimal smooth resolution of the canonical model, and Arakelov's theorem asserts that $K_{\mathcal{G}}^{2}>0$ unless the fibration is isotrivial. The map $\nu$ being ramified only in fibres implies $K_{\widetilde{\mathcal{G}}}=\nu^{*} K_{\mathcal{F}}$, and $(Y, \mathcal{G})$ has canonical singularities so we know that $K_{\widetilde{\mathcal{G}}}$ is big, if the fibration is not isotrivial. Better still bigness is equivalent to strict positivity on the closed cone of nef. $\mathbb{R}$-divisors, and $\nu^{*}: \operatorname{NS}^{1}(\widetilde{X})_{\mathbb{R}} \rightarrow \operatorname{NS}^{1}(\widetilde{Y})_{\mathbb{R}}$ gives an injection of the said cone on $\widetilde{X}$ into that of $\widetilde{Y}$, so if $K_{\widetilde{\mathcal{G}}}$ is big then $K_{\widetilde{\mathcal{F}}}$ is big. Consequently the fibration is isotrivial. Since $K_{\mathcal{G}}$ is Cartier we may appeal to IV.4.4, to find curves $D$ on $\widetilde{Y}$ with $K_{\widetilde{\mathcal{G}}} \cdot D=0=K_{\mathcal{F}} \cdot \nu_{*} D$, so from what we've classified already $X \rightarrow B$ isn't just isotrivial but is a product after an almost étale cover.

We are therefore left to investigate the possibility of $\nu(X, \mathcal{F})=1$, and $\kappa(X, \mathcal{F})=-\infty$ on regular surfaces. This can indeed happen, i.e.

Example IV.5.3. Let $(X, \mathcal{F})$ be a smooth foliated surface with $\nu(X, \mathcal{F})=1$ and $\kappa(X, \mathcal{F})=-\infty$ then $X$ is uniformised by the bi-disc, and indeed the natural foliation on any smooth surface $X$ which is uniformised by the bi-disc is of this type unless it is isogenous to a product of curves.

Rather than offer a proof, let's prepare the way for a singular variant with,

Lemma IV.5.4. Let $(X, \mathcal{F})$ be a foliated smooth surface, and $Z$ a cyclic configuration of smooth rational integral sub-curves or a single rational integral sub-curve with a node such that $K_{\mathcal{F}} \cdot Z_{i}=0$ for any component $Z_{i}$ of $Z$ then $(X, \mathcal{F})$ has canonical singularities in a neighbourhood of $Z$ or $(X, \mathcal{F})$ is a pencil of rational 
curves. In particular in the former case $Z$ is an egl.

Proof. Suppose for simplicity that $Z$ has more than one component. The condition $K_{\mathcal{F}} \cdot Z_{i}=0$ forces the support of $\operatorname{sing}(\mathcal{F})=\operatorname{sing}(Z)$ near $Z$. Now a canonical resolution is obtained by blowing up in points of $\operatorname{sing}(\mathcal{F})(\mathbb{C})$. If we blow up in such a point then either the discrepancy is zero, and there is a new cycle consisting of the proper transform of $Z$ and the exceptional curve which satisfies the same hypothesis, or the discrepancy is negative. If $(X, \mathcal{F})$ is not a rational pencil this discrepancy must be -1 . We therefore obtain a chain of invariant rational curves all of which intersected with the canonical class give 0 , and curves at either end intersecting in -1 . Manifestly, we may assume that the singularities at either end are canonical. Blowing up in any singular point not at the ends doesn't change this configuration if it has zero discrepancy. Otherwise as before the discrepancy must be -1 , and we can take a sub-chain of the same type. However in doing this we eventually arrive at the situation of canonical singularities and so we are done by III.1.2.

Now let us turn to our singular variant,

Example IV.5.5. Let $(X, \mathcal{F})$ be a foliated surface with canonical singularities and underlying space non-singular on which every singularity is contained in an elliptic Gorenstein leaf then $\nu(X, \mathcal{F})=1$ and $\kappa(X, \mathcal{F})=-\infty$ iff $(X, \mathcal{F})$ is an almost étale cover of one of the natural foliations on a Hilbert modular surface (which is not a fibration by algebraic curves).

Proof. Consider a Hilbert-Modular as a quotient of $\mathfrak{H}^{2}$ and consider the foliations arising from the splitting. Now consider the same on the Baily-Borel compactification, and call it $\left(Y_{0}, \mathcal{G}_{0}\right)$ with $\nu:(Y, \mathcal{G}) \rightarrow\left(Y_{0}, \mathcal{G}_{0}\right)$ an almost étale cover which kills the quotient singularities, and $\pi:\left(Y^{\prime}, \mathcal{G}^{\prime}\right) \rightarrow(Y, \mathcal{G})$ a resolution of the cusps, with $Z$ the reduced divisor contracted by $\pi$, then $\Omega_{Y^{\prime}}^{1}(\log Z)=K_{\mathcal{G}} \oplus M$ for some line bundle $M$, and if $(Y, \mathcal{G})$ is not a fibration then the lemma obliges the singularities of either foliation to be canonical, and so both $K_{\mathcal{G}}$ and $M$ are nef. However global 1-forms with log-growth are still closed so the BogomolovCastelnuovo lemma can be generalised to oblige neither $K_{\mathcal{G}}$ nor $M$ to be big. In particular therefore $K_{\mathcal{G}}^{2}=M^{2}=0$. So the previous considerations of our classification imply that $\kappa\left(Y_{0}, \mathcal{G}_{0}\right)=-\infty$. 
For the converse we put $Z$ equal to the union of the e.g.l.'s then the hypothesis force the foliation to be $\log$ smooth, i.e. given by an exact sequence,

$$
0 \rightarrow M \rightarrow \Omega_{X}^{1}(\log Z) \rightarrow K_{\mathcal{F}} \rightarrow 0
$$

Further by a variation of I.3.1 we have $M^{2}=0$, and $K_{\mathcal{F}}$ is certainly nef. We wish to prove, à la IV.1.5, that $M$ is nef. Observe that if for some curve $C$, $M \cdot C<0$ then for a suitable $\varepsilon>0$ we have, $(M-\varepsilon C)^{2}>0$, and $K_{\mathcal{F}} \cdot(M-\varepsilon C)=$ $c_{2}\left(\Omega_{X}^{1}(\log Z)\right)-\varepsilon K_{\mathcal{F}} C$. This will certainly contradict the aforesaid BogomolovCastelnuovo estimates, if we can guarantee $c_{2}\left(\Omega_{X}^{1}(\log Z)\right)=K_{\mathcal{F}} \cdot\left(K_{X}+Z\right)=$ $K_{\mathcal{F}} \cdot K_{X}$ is positive. Suppose otherwise, and observe that by IV.5.2 $X$ is a regular surface, so we have an exact sequence:

$$
0 \rightarrow H^{0}\left(X, K_{X}\right) \rightarrow H^{0}\left(X, \mathcal{O}_{X}\left(K_{X}+Z\right)\right) \rightarrow H^{0}\left(Z, \omega_{Z}\right) \rightarrow 0 .
$$

By hypothesis $H^{0}\left(Z, \omega_{Z}\right) \stackrel{\sim}{\longrightarrow} H^{0}\left(Z, \mathcal{O}_{Z}\right)$ whence $K_{X}+Z$ is effective, and since $K_{\mathcal{F}} \neq 0$ but nef., we must have $\mathcal{O}_{X}\left(K_{X}+Z\right) \stackrel{\sim}{\longrightarrow} \mathcal{O}_{X}$, so by Riemann-Roch, $\chi\left(X, K_{\mathcal{F}}\right)=\chi(X)>0$, since $X$ is regular, which is absurd. Thus $c_{2}\left(\Omega_{X}^{1}(\log Z)\right)>$ 0 , and $M$ is nef. In particular therefore $K_{X}+Z$ is nef. and for any curve $C$, $K_{X}+Z$. $C \geq K_{\mathcal{F}} \cdot C$. However if $C$ is not in the support of $Z$ then $K_{\mathcal{F}} \cdot C=0$ implies $C^{2} \geq 0$, as $(X, \mathcal{F})$ is the minimal resolution of a canonical model, which would force $K_{\mathcal{F}}$ to be effective by the index theorem. Consequently we have,

(a) $\left(K_{X}+Z\right) \cdot C>0$ for all curves $C$ outside the support of $Z$.

Furthermore, $\left(K_{X}+Z\right)^{2}=2 c_{2}\left(\Omega_{X}^{1}(\log Z)\right)>0$ and $K_{\mathcal{F}} \cdot\left(K_{X}+Z\right)>0$, so,

(b) $K_{X}+Z$ is big.

Whence by $[\mathrm{TY}], \Omega_{X}^{1}(\log Z)$ admits a Kahler-Einstein metric, which forces the decomposition $\Omega_{X}^{1}(\log Z)=M \oplus K_{\mathcal{F}}$ and the claimed isomorphism.

We now proceed to show that the above examples are all essentially that may occur, including working in the full generality of Hilbert-Modular surfaces, where the canonical model has quotient singularities arising from contracting $-1 F$ chains. As ever $\rho:(\widetilde{X}, \widetilde{\mathcal{F}}) \rightarrow(X, \mathcal{F})$ is the unique desingularisation of a canonical model, with of course $\nu(X, \mathcal{F})=1$ and $\kappa(X, \mathcal{F})=-\infty$. In particular $\rho^{*} K_{\mathcal{F}}$ is a pseudo-effective $\mathbb{Q}$-Cartier divisor, and so by [D], it admits a 
singular metric, such that if $\rho^{*} \bar{K}_{\mathcal{F}}$ denotes the said divisor with metric then, $c_{1}\left(\rho^{*} \bar{K}_{\mathcal{F}}\right) \geq 0$ where the inequality is understood in the sense of currents. Now $\nu(\mathcal{F})=1$, so $K_{\mathcal{F}}^{2}=0$, and $c_{1}\left(\rho^{*} \bar{K}_{\mathcal{F}}\right) \neq 0$, so if it were defined $c_{1}\left(\rho^{*} \bar{K}_{\mathcal{F}}\right)^{2}=0$. This suggests that we can define a foliation or lamination in some real sense as the kernel of $c_{1}\left(\rho^{*} \bar{K}_{\mathcal{F}}\right)$, which if it were holomorphic would give a transverse holomorphic structure to $\mathcal{F}$. Indeed this is exactly what the Kodaira fibration does except in the case of elliptic fibrations where they coincide - and it's exactly the interplay between the fibration and the foliation that leads to the classification. A priori, the suggestion that the kernel of a smooth positive, yet degenerate, $(1,1)$ form could lead to a holomorphic structure is somewhat outrageous. Such things go under the name of Monge-Ampère foliations, and there is a litany of counterexamples, cf. [Mo]. However if $\overline{\bar{K}}_{\mathcal{F}}$ were another metricisation, and everything made sense, then $c_{1}\left(\rho^{*} \bar{K}_{\mathcal{F}}\right) \cap c_{1}\left(\rho^{*} \overline{\bar{K}}_{\mathcal{F}}\right)=0$, so such a kernel ought to be unique, whence morally at least continuous. Indeed we can even make a good moral argument for holomorphicity. So let's do this, starting with,

Definition IV.5.6. Let $(X, \mathcal{F})$ be a foliation by curves on a non-singular variety $X$ and $\bar{L}=(L,\|\|)$ a line bundle with metric then we define the Chern class of $L$ along $\mathcal{F}$ to be,

$$
c_{\mathcal{F}}(\bar{L})=\frac{-1}{2 \pi \sqrt{-1}} \bar{\partial}\left(\partial_{\mathcal{F}} \log \|s\|^{2}\right) \otimes \partial_{\mathcal{F}}^{\vee}
$$

where $s$ is a local section of $L$, and $\partial_{\mathcal{F}}$ a local generator of $T_{\mathcal{F}}$. If the metric is smooth $c_{\mathcal{F}}$ takes values in $A_{X}^{0,1} \otimes K_{\mathcal{F}}$, or more generally $D_{X}^{0,1} \otimes K_{\mathcal{F}}$, i.e. distributions, or whatever according to the regularity class. Indeed at the level of Dolbeault cohomology $c_{\mathcal{F}}(\bar{L})$ is the restriction to $H_{\bar{\partial}}^{0,1}\left(K_{\mathcal{F}}\right)$ of $c_{1}(\bar{L})$ in $H_{\bar{\partial}}^{1,1}$.

Now consider a smooth point of the foliation on $(X, \mathcal{F})$ (let's say we're back to surfaces again for simplicity) then we can find coordinates $x, y$ with $\partial_{\mathcal{F}}=\partial / \partial x$ a local generator for the foliation, and whence a non-canonical identification of $d x d \bar{x} d y d \bar{y}$ with $d \bar{x} d y d \bar{y} \otimes \partial_{\mathcal{F}}^{\vee}$ with respect to which,

$$
c_{1}(\bar{L})^{2}=1 / 2 c_{1}(\bar{L}) \wedge c_{\mathcal{F}}(\bar{L})
$$

modulo the equation making sense, e.g. $c_{1}(\bar{L})$ having $L_{2}$-coefficients. One can make a more complicated formula at the singular points, but the important thing is that a singularity makes the right hand side smaller. 
To fix ideas let's suppose momentarily that $(\tilde{X}, \widetilde{\mathcal{F}})=(X, \mathcal{F})$, and that $K_{\mathcal{F}}$ admits a smooth positive semi-definite metric then certainly, $c_{1}\left(\bar{K}_{\mathcal{F}}\right) \wedge c_{\mathcal{F}}\left(\bar{K}_{\mathcal{F}}\right)=$ 0 everywhere, and the 1 -form defining the hoped for transverse structure is the conjugate of $c_{\mathcal{F}}\left(\bar{K}_{\mathcal{F}}\right)$. We can use the Bochner technique to show holomorphicity. Siu's $\partial \bar{\partial}$ formulation, [Si2], is convenient. Specifically if $e^{-2 \varphi}$ is a local weight for the metric then since $c_{\mathcal{F}}\left(\bar{K}_{\mathcal{F}}\right)$ is $\bar{\partial}$-closed,

$$
\begin{aligned}
\frac{\sqrt{-1}}{2 \pi} \partial \bar{\partial}\left(e^{-2 \varphi} c_{\mathcal{F}}\left(\bar{K}_{\mathcal{F}}\right) \wedge \overline{c_{\mathcal{F}}\left(\bar{K}_{\mathcal{F}}\right)}\right)= & \frac{-\sqrt{-1}}{2 \pi} \partial\left\{e^{-2 \varphi} c_{\mathcal{F}}\left(\bar{K}_{\mathcal{F}}\right) \overline{\nabla c_{\mathcal{F}}\left(\bar{K}_{\mathcal{F}}\right)}\right\} \\
= & \frac{-\sqrt{-1}}{2 \pi} e^{-2 \varphi} \nabla c_{\mathcal{F}}\left(\overline{\left.K_{\mathcal{F}}\right) \wedge} \overline{\nabla c_{\mathcal{F}}\left(\bar{K}_{\mathcal{F}}\right)}\right. \\
& +e^{-2 \varphi} c_{\mathcal{F}}\left(\bar{K}_{\mathcal{F}}\right) \wedge \overline{c_{\mathcal{F}}\left(\bar{K}_{\mathcal{F}}\right)} c_{1}\left(\bar{K}_{\mathcal{F}}\right) .
\end{aligned}
$$

So if $\nu(\mathcal{F})=1$, the curvature term should vanish, the left hand side integrates out to zero and we obtain,

$$
\int_{X} e^{-2 \varphi} \nabla c_{\mathcal{F}}\left(\bar{K}_{\mathcal{F}}\right) \wedge \overline{\nabla c_{\mathcal{F}}\left(\bar{K}_{\mathcal{F}}\right)}=0 .
$$

On the other hand, $c_{\mathcal{F}}\left(\bar{K}_{\mathcal{F}}\right) \wedge c_{1}\left(\bar{K}_{\mathcal{F}}\right)=0$, from which $\nabla c_{\mathcal{F}}\left(\bar{K}_{\mathcal{F}}\right) \wedge c_{1}\left(\bar{K}_{\mathcal{F}}\right)=0$. Now suppose we're somewhere $p$, say, on $X$ where $c_{1}\left(\bar{K}_{\mathcal{F}}\right)$ doesn't vanish and write $c_{1}\left(\bar{K}_{\mathcal{F}}\right)$ at $p$ as $\frac{d x d \bar{x}}{-2 \pi \sqrt{-1}}$, with $\frac{d x d \bar{x}+d y d \bar{y}}{-2 \pi \sqrt{-1}}$ the Kahler form then,

(a) $\nabla c_{\mathcal{F}}\left(\bar{K}_{\mathcal{F}}\right) \wedge c_{1}\left(\bar{K}_{\mathcal{F}}\right)=0$ implies that there is no $d y d \bar{y}$ term in $\nabla c_{\mathcal{F}}\left(\bar{K}_{\mathcal{F}}\right)$.

(b) Which in turn says, $e^{-2 \varphi} \nabla c_{\mathcal{F}}\left(\bar{K}_{\mathcal{F}}\right) \wedge \overline{\nabla c_{\mathcal{F}}\left(\bar{K}_{\mathcal{F}}\right)}>0$ at $p$, unless $\nabla c_{\mathcal{F}}\left(\bar{K}_{\mathcal{F}}\right)$ only has a $d x d \bar{x}$ term.

Putting everything together implies $c_{\mathcal{F}}\left(\bar{K}_{\mathcal{F}}\right) \wedge \nabla c_{\mathcal{F}}\left(\bar{K}_{\mathcal{F}}\right)=0$, and this is the holomorphicity equation. So what exactly are the problems. Unfortunately they are legion, namely:

(i) The Bochner formula appears to be unjustifiable without some sort of regularity for the local weight $\varphi$. At the very least it looks like we need that it's in the Sobolev space $W_{2,2}$ (here $W_{p, q}$ means $p^{\text {th }}$-derivatives being $L_{q}$ ) and maybe even $W_{4,2}$, whereas general nonsense only gives $W_{1,2-\varepsilon}$.

(ii) We need to understand where $c_{\mathcal{F}}\left(\bar{K}_{\mathcal{F}}\right)$ (equivalently $c_{1}\left(\bar{K}_{\mathcal{F}}\right)$ ) vanishes. Indeed even in a smooth situation if $c_{\mathcal{F}}\left(\bar{K}_{\mathcal{F}}\right)=\bar{f} d \bar{x}+\bar{g} d \bar{y}$, say, in local coordinates $x, y$ 
and $g \neq 0$ the holomorphicity equation gives $\bar{\partial}(f / g)=0$. However if both $f$ and $g$ are zero, there's some sort of pole that needs to be controlled.

(iii) The holomorphicity equation, $c_{\mathcal{F}}\left(\bar{K}_{\mathcal{F}}\right) \wedge \nabla c_{\mathcal{F}}\left(\bar{K}_{\mathcal{F}}\right)=0$ is non-linear, and as Gromov pointed out to me it's invariant under bumping, so it's trivial to make sequences of $(1,0)$ forms $\omega_{n}$ converging to some distribution $\omega$ with $\omega_{n} \wedge \bar{\partial} \omega_{n} \rightarrow 0$ such that $\omega=0$ does not define a field of holomorphic lines.

(iv) Even if all went well, and the kernel was a well defined holomorphic foliation, we have to exclude the possibility that it coincides with $\mathcal{F}$.

Leaving (iv) apart, all of which certainly implies that we're not going to get anywhere without some a priori regularity. Returning then to the general situation where $\rho:(\widetilde{X}, \widetilde{\mathcal{F}}) \rightarrow(X, \mathcal{F})$ is the minimal smooth resolution of the canonical model and $\nu(\mathcal{F})=1$, but $\kappa(\mathcal{F})=-\infty$, the following observation is fundamental,

Lemma IV.5.7. Notations and hypothesis as above then $\forall x \in \widetilde{X}$, the Lelong number $\nu\left(x, c_{1}\left(\rho^{*} \bar{K}_{\mathcal{F}}\right)\right)=0$.

Proof. Suppose otherwise. Then for some $\varepsilon>0$ the set,

$$
E_{\varepsilon}=\left\{x \in \widetilde{X} \mid \nu\left(x, c_{1}\left(\rho^{*} \bar{K}_{\mathcal{F}}\right)\right) \geq \varepsilon\right\} \neq \varphi
$$

and by the celebrated theorem of Siu, cf. [Si], it is in fact a closed analytic subset of $\widetilde{X}$. Whence there are two possibilities $\operatorname{dim} E_{\varepsilon}=1$ or $\operatorname{dim} E_{\varepsilon}=0$. Suppose the former then by Siu's decomposition theorem, cf. op.cit., there are real numbers $\nu_{1}, \ldots, \nu_{k} \geq \varepsilon$, curves $C_{1}, \ldots, C_{k}$ and a positive $(1,1)$ current $T_{\varepsilon}$ such that,

$$
c_{1}\left(\rho^{*} \bar{K}_{\mathcal{F}}\right)=\sum_{i=1}^{k} \nu_{i} C_{i}+T_{\varepsilon}
$$

where $C_{i}$ denotes in this formula the current of integration over the curve. Now use duality to project everything into $\mathrm{NS}_{1}(\widetilde{X})_{\mathbb{R}}$, lowering the $\nu_{i}$ a little to $\nu_{i}^{\prime}$ to ensure that they are a rational we obtain a pseudo-effective $\mathbb{Q}$-divisor $T$, such that,

$$
\rho^{*} K_{\mathcal{F}}=\sum_{i=1}^{k} \nu_{i}^{\prime} C_{i}+T
$$


at which point we may apply Zariski decomposition to $T$, and use the index theorem to deduce that $\rho^{*} K_{\mathcal{F}}$ is represented by a positive cycle. Since without loss of generality $\widetilde{X}$ is regular, this is absurd.

Otherwise $E_{\varepsilon}$ must have dimension 0 , and indeed this must hold $\forall \varepsilon>0$, so the subset, $\left\{x \in \widetilde{X} \mid \nu\left(x, c_{1}\left(\rho^{*} \bar{K}_{\mathcal{F}}\right)\right)>0\right\}$ is countable. However by [D], we must have in this situation,

$$
c_{1}\left(\rho^{*} \bar{K}_{\mathcal{F}}\right)^{2} \geq \sum_{x \in \widetilde{X}} \nu\left(x, c_{1}\left(\rho^{*} \bar{K}_{\mathcal{F}}\right)\right)^{2}
$$

which establishes the lemma.

Although the vanishing of Lelong numbers represents some sort of regularity it's unfortunately very far from forcing the local weight $\varphi$ of the metric to enjoy anything near the kind of a priori regularity that would be required to force the Bochner type argument through. Consequently, following Brunella, [Br4], \& [Br5], we look towards the obvious choice for metricising $K_{\mathcal{F}}$, namely the leaf wise Poincaré metric. This is most naturally defined on the Gorenstein covering tack $\mathcal{X} \rightarrow X$, where over the smooth locus $\mathcal{U} \subset \mathcal{X}$ of $\mathcal{F}$ with $\partial$ a local trivialisation of $T_{\mathcal{F}}$ this is defined at $x \in \mathcal{U}$ by,

$$
e^{\varphi(x)}=\|\partial\|(x)=\inf \left\{\frac{1}{|R|}: f:(\Delta, 0) \rightarrow(\mathcal{U}, x), f_{*}\left(\frac{\partial}{\partial z}\right)=R \partial\right\}
$$

For general nonsense reasons (i.e. everything which is Stein has a Stein neighbourhood) the function $\varphi$ is upper semi-continuous. Indeed, since we would like $\varphi$ to be pluri-subharmonic, the need for upper semi-continuity precisely demands, [Br5], that the non-scheme like terminal points of $\mathcal{X}$ should be treated as being smooth points of $\mathcal{F}$, which, of course, they are in the stack sense. If one doesn't have recourse to $\mathcal{X}$, then certainly $\varphi$ can be defined on $X$ by way of prescribing the ramification of discs mapping to terminal points of $\mathcal{F}$ where $X$ is singular, with the ramification given by the determinants of the contracted $-1 F$ chains, but this is somewhat artificial and fails to convey what is going on.

A priori, the Poincaré metric is only defined on $\mathcal{U}$, but the first of the following two facts permits its extension over the singularities of $\mathcal{F}$,

Facts IV.5.8. The Poincaré metric has the following critical properties, 
(a) $([\mathrm{Br} 5])$ The local weight $\varphi$ is pluri-subharmonic.

(b) ([M4] V.5.1) If $K_{\tilde{X}} \cdot \rho^{*} K_{\mathcal{F}}>0$ then $\varphi$ is locally bounded outside of finitely many invariant algebraic curves.

Plainly, in our current context this is just what we need, since we know that $q(\widetilde{X})=0$, and so $K_{\tilde{X}} \cdot \rho^{*} K_{\mathcal{F}}>0$ by Riemann-Roch. As it happens, one can strengthen (b) to continuous, cf. op. cit., which for convenience we'll suppose. Further in this metricisation the kernel of $c_{1}\left(\rho^{*} \bar{K}_{\mathcal{F}}\right)$ has sense, viz:

Fact IV.5.8.bis. ([Br5]) Suppose that globally, $K_{\mathcal{F}}^{2}=0$ and $\varphi$ is locally bounded around $x \in \mathcal{U}$, then, around $x, c_{1}\left(\bar{K}_{\mathcal{F}}\right)$ is absolutely continuous.

Now we have a well defined kernel, and observe quite generally, in the notations of the proof that for any metricised line bundle $\bar{L}$, with $c_{1}(\bar{L})$ absolutely continuous and local weight $\psi$ with $\psi_{x \bar{x}} \neq 0$,

$$
c_{1}(\bar{L})=\frac{\pi \sqrt{-1}}{\psi_{x \bar{x}}} \overline{c_{\mathcal{F}}(\bar{L})} \wedge c_{\mathcal{F}}(\bar{L})+\frac{\sqrt{-1}}{\pi} \frac{\Delta(\psi)}{\psi_{x \bar{x}}} d y d \bar{y}
$$

where $\Delta(\psi)=\psi_{x \bar{x}} \psi_{y \bar{y}}-\left|\psi_{x \bar{y}}\right|^{2}$, and the formula is understood as a pointwise identity of $L_{1}$-functions. Applying this to $\bar{K}_{\mathcal{F}}$ with the Poincaré metric gives, off the excluded algebraic set,

$$
c_{1}\left(\bar{K}_{\mathcal{F}}\right)=\pi \sqrt{-1} e^{-2 \varphi} \overline{c_{\mathcal{F}}\left(\bar{K}_{\mathcal{F}}\right)} \wedge c_{\mathcal{F}}\left(\bar{K}_{\mathcal{F}}\right) .
$$

Consequently if it were defined the Bochner formula would give a pointwise identity; there being no need to integrate out the left hand side,

$$
e^{-2 \varphi} \nabla c_{\mathcal{F}}\left(\bar{K}_{\mathcal{F}}\right) \wedge \overline{\nabla c_{\mathcal{F}}\left(\bar{K}_{\mathcal{F}}\right)}=0
$$

In terms of local coordinates this amounts to, $\frac{\partial}{\partial \bar{x}}\left(\frac{\varphi_{y \bar{x}}}{\varphi_{x \bar{x}}}\right)=0$, which in the presence of sufficient regularity is the standard holomorphicity equation for MongeAmpère foliations. The transverse holomorphicity usually being trivial thanks to the identity $\frac{\partial}{\partial \bar{y}}\left(\frac{\varphi_{y \bar{x}}}{\varphi_{x \bar{x}}}\right)=\frac{\varphi_{x \bar{y}}}{\varphi_{x \bar{x}}} \frac{\partial}{\partial \bar{x}}\left(\frac{\varphi_{y \bar{x}}}{\varphi_{x \bar{x}}}\right)$ which follows from $\Delta(\varphi)=0$. Our strategy then will simply be to push this analysis through by way of appropriate regularisation. Before getting under way, however, let's observe that if we succeed then we'll get our desired transverse foliation $\mathcal{G}$ on all of $X$. Indeed away from 
the singularities and the algebraic invariant curves through them $\varphi_{x \bar{x}}^{-1}=e^{-2 \varphi}$ is still continuous, and $\mathcal{G}$ is defined by the 1 -form,

$$
\frac{\varphi_{y \bar{x}}}{\varphi_{x \bar{x}}} d y+d x=0
$$

so it's certainly transverse to $\mathcal{F}$ and not equal to it. Now consider a suitably small polydisc $\Delta$ meeting an algebraic invariant curve but not containing the singularities, then we certainly still have foliation coordinates $x, y$, with $y=$ const., the foliation, and on $\Delta^{*}=\Delta \backslash\{$ said curve $\}, \mathcal{G}$ is still given by the same equation. Better still on $\Delta^{*}, e^{-2 \varphi}\left|\varphi_{y \bar{x}}\right|^{2}=\varphi_{y \bar{y}}$ and $\varphi$ is $L_{p}$ for all $p<\infty$ by the vanishing of the Lelong numbers so if $d \mu$ is Lebesgue measure,

$$
\begin{aligned}
\left(\int_{\Delta^{*}}\left|\frac{\varphi_{y \bar{x}}}{\varphi_{x \bar{x}}}\right| d \mu\right)^{2} \leq & \left(\int_{\Delta^{*}}\left|\varphi_{y \bar{x}}\right| e^{-2 \varphi} d \mu\right)\left(\int_{\Delta^{*}} e^{-2 \varphi} d \mu\right) \\
& \leq \varphi_{y \bar{y}}\left(\mathbb{I}_{\Delta}\right) \int_{\Delta^{*}} e^{-2 \varphi} d \mu<\infty
\end{aligned}
$$

Whence if $\frac{\varphi_{y \bar{x}}}{\varphi_{x \bar{x}}}$ is holomorphic on $\Delta^{*}$, it's at worst meromorphic on $\Delta$ with a simple pole along the curve in question. Consequently $\mathcal{G}$ would be a well defined foliation on $X$ in codimension 1 , and since $X$ is normal, well defined everywhere.

So let's justify the Bochner technique. Although everything is local it's convenient to keep the curvature notation $c_{1}, c_{\mathcal{F}}, \nabla$ etc., but this time we'll write $c_{1}(\psi)$, etc. for $\psi$ a local weight, corresponding to a metric $e^{-2 \psi}$ on $K_{\mathcal{F}}$. Furthermore $f$ will be a positive compactly supported function on a polydisc $\Delta$ not containing the singularities nor meeting an algebraic invariant curve through them. Observe, moreover, that the proof of IV.5.8. bis gives quite generally that if $\varphi_{k} \rightarrow \varphi$ monotone, then, $c_{1}\left(\varphi_{k}\right) \wedge c_{1}(\varphi) \underset{w}{\longrightarrow} 0$. On the other hand we have,

$$
c_{1}\left(\varphi_{k}\right)=\pi \sqrt{-1} \frac{\overline{c_{\mathcal{F}}\left(\varphi_{k}\right)} \wedge c_{\mathcal{F}}\left(\varphi_{k}\right)}{\varphi_{k, x \bar{x}}}+\frac{\sqrt{-1}}{\pi} \frac{\Delta\left(\varphi_{k}\right)}{\varphi_{k, x \bar{x}}} d y d \bar{y} .
$$

Naturally we introduce $\lambda(\psi)=\frac{\psi_{x \bar{y}}}{\psi_{x \bar{x}}}$, for any $\psi$, so that our previous observation is equivalent to,

$$
\varphi_{x \bar{x}} \varphi_{k, x \bar{x}}\left|\lambda(\varphi)-\lambda\left(\varphi_{k}\right)\right|^{2} \underset{w}{\longrightarrow} 0 \text { and } \frac{\varphi_{x \bar{x}}}{\varphi_{k, x \bar{x}}} \Delta\left(\varphi_{k}\right) \underset{w}{\longrightarrow} 0 .
$$

In particular for $\varphi_{k} \rightarrow \varphi$ monotone obtained via regularising kernels in the usual way, $\varphi_{k, x \bar{x}} \geq e^{2 \varphi_{k}} \geq e^{2 \varphi}$ so $\lambda\left(\varphi_{k}\right) \underset{w}{\longrightarrow} \lambda(\varphi)$ in $L_{2}$. Now consider the 
connection $\nabla_{k}$ on $K_{\mathcal{F}}$ corresponding to the metric $\varphi_{x \bar{x}}^{k}=\left\|\frac{\partial}{\partial x}\right\|^{2}$. Then,

$$
\begin{aligned}
\frac{\sqrt{-1}}{2 \pi} \int_{\Delta} f \partial \bar{\partial}\left\{\frac{c_{\mathcal{F}}\left(\varphi_{k}\right) \overline{c_{\mathcal{F}}\left(\varphi_{k}\right)}}{\varphi_{k, x \bar{x}}}\right\}= & \frac{-\sqrt{-1}}{2 \pi} \int_{\Delta} \frac{f}{\varphi_{k, x \bar{x}}} \nabla_{k} c_{\mathcal{F}}\left(\varphi_{k}\right) \overline{\nabla_{k} c_{\mathcal{F}}\left(\varphi_{k}\right)} \\
& +\int_{\Delta} f \cdot c_{1}\left(\log \varphi_{k, x \bar{x}}\right) \frac{c_{\mathcal{F}}\left(\varphi_{k}\right) \overline{c_{\mathcal{F}}\left(\varphi_{k}\right)}}{\varphi_{k, x \bar{x}}}
\end{aligned}
$$

Now $\varphi_{k, x \bar{x}}^{-1} c_{\mathcal{F}}\left(\varphi_{k}\right) \overline{c_{\mathcal{F}}\left(\varphi_{k}\right)}=\frac{1}{\pi^{2}} \varphi_{k, x \bar{x}}\left(d x d \bar{x}+\overline{\lambda\left(\varphi_{k}\right)} d y d \bar{x}+\lambda\left(\varphi_{k}\right) d x d \bar{y}\right.$ $\left.+\left|\lambda\left(\varphi_{k}\right)\right|^{2} d y d \bar{y}\right)$, and $\varphi_{k, x \bar{x}} \rightarrow \varphi_{x \bar{x}}$ in $L_{\infty}$ while $\lambda\left(\varphi_{k}\right) \rightarrow \lambda$ in $L_{2}$, so the whole thing goes weakly to, $\varphi_{x \bar{x}}^{-1} c_{\mathcal{F}}(\varphi) \overline{c_{\mathcal{F}}(\varphi)}$ which is closed. So in fact,

$$
\frac{1}{2 \pi} \int_{\Delta} \frac{f}{\varphi_{k, x \bar{x}}}\left|\frac{\partial \lambda\left(\varphi_{k}\right)}{\partial x}\right|^{2} d \mu=\lim _{k} \sqrt{-1} \int_{\Delta} f c_{1}\left(\log \varphi_{k, x \bar{x}}\right) \frac{\overline{c_{\mathcal{F}}\left(\varphi_{k}\right)} c_{\mathcal{F}}\left(\varphi_{k}\right)}{\varphi_{k, x \bar{x}}} .
$$

To estimate the integral on the right, observe that $\log \varphi_{k, x \bar{x}}$ is monotone decreasing to $\varphi$, so $c_{1}\left(\log \varphi_{k, x \bar{x}}\right) \wedge c_{1}(\varphi) \underset{w}{\longrightarrow} 0$. Defining a curvature function, $G(\psi)=e^{-2 \psi} \frac{\partial^{2} \psi}{\partial x \partial \bar{x}}$ for any suitable $\psi$ we can write this as,

$$
e^{2 \varphi} G\left(\log \varphi_{k, x \bar{x}}\right) \varphi_{k, x \bar{x}}\left|\lambda(\varphi)-\lambda\left(\log \varphi_{k, x \bar{x}}\right)\right|^{2} \underset{w}{\longrightarrow} 0
$$

and

$$
\frac{e^{2 \varphi}}{\varphi_{k, x \bar{x}} G\left(\log \varphi_{k, x \bar{x}}\right)} \Delta\left(\log \varphi_{k, x \bar{x}}\right) \underset{w}{\longrightarrow} 0
$$

while the integral we're seeking to estimate is,

$$
\int_{\Delta} f G\left(\log \varphi_{k, x \bar{x}}\right)\left|\varphi_{k, x \bar{x}}\right|^{2}\left|\lambda\left(\varphi_{k}\right)-\lambda\left(\log \varphi_{k, x \bar{x}}\right)\right|^{2} \frac{d \mu}{\pi}+\int_{\Delta} f \frac{\Delta\left(\log \varphi_{k, x \bar{x}}\right)}{G\left(\log \varphi_{k, x \bar{x}}\right)} \frac{d \mu}{\pi}
$$

the latter integral easily tends to zero by what we already have, and the former will also easily be so on obtaining an upper bound for the curvature. Denoting by $\Phi_{k}(x)=\sup _{x \in \Delta} \sup \left\{\varphi(y) \mid \rho_{k}(|x-y|) \neq 0\right\}$ where $\rho_{k}$ is the symmetric bump function giving the convolution, we have,

$$
\left|G\left(\log \varphi_{k, x \bar{x}}\right)\right| \leq \frac{2 e^{2 \Phi_{k}}}{e^{4 \varphi}}\left\|\varphi_{x}\right\|_{L_{2}}+\frac{e^{4 \Phi_{k}}}{e^{4 \varphi}}
$$

since $\left|\varphi_{x \bar{y}}\right|^{2}=\varphi_{x \bar{x}} \varphi_{y \bar{y}}$, the Garding inequality implies that $\varphi_{x}$ is $L_{2}$ so indeed the curvature is bounded above, and whence,

$$
\frac{\partial}{\partial x}(\lambda(\varphi))(f)=\lim _{k} \int \lambda\left(\varphi_{k}\right) f_{x} d \mu=\lim _{k} \int \frac{\partial}{\partial x}\left(\lambda\left(\varphi_{k}\right)\right) f d \mu=0 .
$$


For the transverse direction the basic fact is that $c_{1}(\varphi)=\pi \sqrt{-1} \overline{c_{\mathcal{F}}(\varphi)} \wedge c_{\mathcal{F}}(\varphi) e^{-2 \varphi}$ is closed, which in terms of our test function $f$ gives two sets of equations,

$$
\int_{\Delta} f_{y} \lambda(\varphi) e^{2 \varphi}-f_{x}|\lambda|^{2} e^{2 \varphi} d \mu=0 \quad \text { and } \quad \int_{\Delta} f_{y} e^{2 \varphi}-e^{2 \varphi} \overline{\lambda(\varphi)} f_{x}=0 .
$$

Now for notational convenience put $\lambda=\lambda(\varphi)$ and take regularising convolutions $\varphi_{k} \rightarrow \varphi, \lambda_{m} \rightarrow \lambda, \bar{\lambda}_{n} \rightarrow \bar{\lambda}$ (n.b. $\lambda_{m} \neq \lambda\left(\varphi_{m}\right)$, it's just a convolution of $\lambda$ ) then possibly after subsequencing the $1^{\text {st }}$ of these converges weakly in $L_{\infty}$, the other two weakly in $L_{2}$. Certainly $\left(\lambda_{m}\right)_{x}=0$, and the following limits irrespective of the ordering in $k, m$, and $n$ go to zero, viz,

$$
\int\left(\lambda_{m} e^{2 \varphi_{k}}\right)_{y} f-\left(\lambda_{m} \bar{\lambda}_{n} e^{2 \varphi_{k}}\right)_{x} f d \mu \text { and } \int\left(e^{2 \varphi_{k}}\right)_{y} f-\left(e^{2 \varphi_{k}} \bar{\lambda}_{n}\right)_{x} f .
$$

However, $\left(\lambda_{m} e^{2 \varphi_{k}}\right)_{y} f-\left(\lambda_{m} \bar{\lambda}_{n} e^{2 \varphi_{k}}\right)_{x} f=\left(\lambda_{m}\right)_{y} e^{2 \varphi_{k}} f+\lambda_{m} f\left(e^{2 \varphi_{k}}\right)_{y}-\lambda_{m} f\left(\bar{\lambda}_{n} e^{2 \varphi_{k}}\right)_{x}$, so fixing $m$ and replacing $f$ by $\lambda_{m} f$ in the second integral gives,

$$
\lim _{m \rightarrow \infty} \int_{\Delta} e^{2 \varphi} f\left(\lambda_{m}\right)_{y} d \mu=0 .
$$

Now since $\varphi$ is continuous and psh, we may subsequence so that $e^{2 \varphi_{k}}$ has a weak limit in $W_{1,2}$ say $e^{2 \varphi}$ by a possible minor abuse of notation, and $e^{2 \varphi_{k}} f \underset{w}{\longrightarrow} e^{2 \varphi} f$ again in $W_{1,2}$ so,

$$
\int_{\Delta} \lambda\left(e^{2 \varphi} f\right)_{y} d \mu=0
$$

Given that $f$ is arbitrary, the space of functions $e^{2 \varphi} f$ is dense in $W_{1,2}$ so indeed $\lambda_{y}=0$, and $\varphi_{x \bar{x}}^{-1} \varphi_{y \bar{x}}$ is holomorphic on $\Delta$.

At this point we have found our foliation $\mathcal{G}$, and we'll work on the minimal smooth resolution $\rho:(\widetilde{X}, \widetilde{\mathcal{F}}) \rightarrow(X, \mathcal{F})$. Denote by $D$ the tangency divisor between $\widetilde{\mathcal{F}}$ and $\widetilde{\mathcal{G}}$ which we've shown to be $\widetilde{\mathcal{F}}$ invariant with simple poles, so it is the same for $\widetilde{\mathcal{G}}$ and $\Omega_{\widetilde{X}}^{1}(\log D)$ splits as $K_{\widetilde{\mathcal{F}}} \oplus K_{\widetilde{\mathcal{G}}}$. Now denote by $N_{\widetilde{\mathcal{F}}}$ and $N_{\widetilde{\mathcal{G}}}$ the respective logarithmic conormal bundles with $s_{\widetilde{\mathcal{F}}}(C)$, resp. $s_{\widetilde{\mathcal{G}}}(C)$ the Segre class along $\operatorname{sing}(\mathcal{F})$, resp. $\operatorname{sing}(\mathcal{G})$, of any curve while for $C \in D$, put $s(C, D)=D \cdot C-C^{2}$, i.e. the number of curves in $D$ meeting $C$, then, for $C \subset|D|$, we have,

$$
K_{\widetilde{\mathcal{F}}} \cdot C=-s_{\widetilde{\mathcal{G}}}(C)+s(C, D) \leq 0
$$

and similarly for $K_{\widetilde{\mathcal{G}}} \cdot C$, irrespective of whether the latter has canonical singularities or not. To conclude that we really do have a Hilbert-Modular surface 
with the natural foliations there on, the first step is to show that $\widetilde{\mathcal{G}}$ has canonical singularities. Observe any singularity of $\widetilde{\mathcal{G}}$ is contained in a curve $C \subset|D|$, so the question is local about $D$. Furthermore $K_{\widetilde{\mathcal{F}}} \cdot C \leq 0$ for all $C \subset|D|$, so not only is $D$ contracted by $\rho$, but it cannot be a $D_{n}$, so a connected component $\Gamma$ can only be,

\section{Cases IV.5.9.}

(a) A (not necessarily maximal) $-1 F$ chain for $\widetilde{\mathcal{F}}$.

(b) A (not necessarily maximal) chain of $-2 F$ curves for $\widetilde{\mathcal{F}}$.

(c) A cycle of $-2 F$ curves (or a rational curve with a node) for $\widetilde{\mathcal{F}}$.

Now as in the proof of the canonical model theorem the basic fact is that for any foliation with canonical singularities a chain of curves starting and ending in a $-1 F$ curve which are joined by $-2 F$ curves cannot be contractible, or even easier an invariant rational curve intersecting the canonical in -2 isn't contractible either. So consider firstly case (c), for any $C \subset \Gamma, s(C, D)=s_{\widetilde{\mathcal{G}}}(C)=2$, so $K_{\widetilde{\mathcal{G}}} \cdot C=0$, and the singularities of $\widetilde{\mathcal{G}}$ coincide with those of $\Gamma$. Suppose, therefore, that one of these is non-canonical. Blow up in it and call the exceptional divisor $E$, then either it's discrepant or not. If it's not $K_{\widetilde{\mathcal{G}}} \cdot E=0$ and $E^{2}=-1$, so $E$ is invariant, with $s_{\widetilde{\mathcal{G}}}(E)=\widetilde{\Gamma} \cdot E=2$, where $\widetilde{\Gamma}$ is the proper transform of $\Gamma$, and we still have a splitting, i.e. we've changed nothing, so we might as well say that we've a priori blown up sufficiently in order to guarantee negative discrepancy. In which case on blowing up $\widetilde{\Gamma}$ is two $-1 F$ curves for $\widetilde{\mathcal{G}}$ joined by a (possibly empty) chain of $-2 F$ curves for $\widetilde{\mathcal{G}}$ or an invariant rational curve intersecting the canonical in -2 . Of course we still may not have canonical singularities, but the singularities of the induced foliation, which we'll still call $\widetilde{\mathcal{G}}$ to avoid complicating the notation, still coincide with those of $\widetilde{\Gamma}$. Blowing up in one of those we either keep a chain with the same properties in the non-discrepant case, or split into two disjoint chains with the same property. In any case we eventually contradict the contractibility of $D$. Case (b) automatically reduces to the latter part of case (c), while for case (a) the $-1 F$ chain for $\widetilde{\mathcal{F}}$ is also a $-1 F$ chain for $\widetilde{\mathcal{G}}$ but in the opposite sense, i.e. in the following Dynkin diagram for $\Gamma$, the $K_{\widetilde{\mathcal{F}}}$ intersection numbers are on the bottom, those for $\widetilde{\mathcal{G}}$ on the top, 


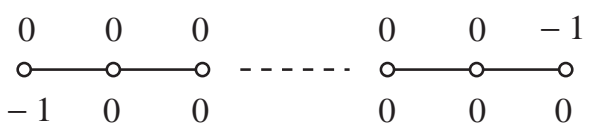

Here the $\widetilde{\mathcal{G}}$ and $\Gamma$ singularities may not a priori coincide on the curve on the left, say by virtue of a singularity $z \in \operatorname{sing}(\widetilde{\mathcal{G}}) \backslash \Gamma$. However blowing up in $z$ in the non-discrepant case only adds a node to the left, the pattern of 0's and -1's being the same for the induced foliation, so we can assume that we're only one blow up away, and we again conclude by the latter discussion for (c).

Now supposing that $\widetilde{\mathcal{G}}$ is not a rational fibration, the next step is to consider what happens to curves $\Gamma$ contracted by a canonical model map $\sigma:(\widetilde{X}, \widetilde{\mathcal{G}}) \rightarrow$ $\left(X^{\prime}, \mathcal{G}^{\prime}\right)$ for $\widetilde{\mathcal{G}}$. As ever we proceed by cases,

Cases IV.5.10. (a) $\Gamma$ is a maximal $-1 F$ chain for $\widetilde{\mathcal{G}}$. Ordering the curves in the chain in the usual way call them $C_{1}, \ldots, C_{n}$. Then we have the sub-cases,

(i) $C_{1} \subset|D|$, and let $C_{1}, \ldots, C_{k}$ be the maximal sub-chain of $T$ in $D$ connected to $C_{1}$. Suppose $k<n$, so in particular $n \geq 2$. Now suppose further $k+1<n$, then $C_{k+2} \subset|D|$, and $K_{\widetilde{\mathcal{F}}} \cdot C_{k+2}=s\left(C_{k+2}, D\right)-2$. Further there is a maximal sub-chain of $T$ in $D$ connected to $C_{k+2}$, say $C_{k+2}, \ldots, C_{k+m}, m \geq 2$. If $m=2$, $s\left(C_{k+2}, D\right)=0$, and $C_{k+2}$ isn't contractible or $m \geq 3$ implying $K_{\tilde{\mathcal{F}}} \cdot C_{k+2}=$ $K_{\widetilde{\mathcal{F}}} \cdot C_{k+m}=-1$ and $C_{k+2}, \ldots, C_{k+m}$ non-contractible, both of which are absurd. Consequently $n=k+1$, and $\left(K_{\widetilde{\mathcal{F}}}+C_{n}\right) \cdot C_{n}=-s_{\mathcal{G}}\left(C_{n}\right)+D \cdot C_{n}=-1$, so by adjunction $C_{n}$ is $\widetilde{\mathcal{F}}$ invariant, so is tangent to $\widetilde{\mathcal{F}}$ and $\widetilde{\mathcal{G}}$, i.e. $C_{n} \subset|D|$, contrary to hypothesis, implying thus that $T$ is a component of $D$.

(ii) $C_{1}$ is not in $D$. Consequently $n \geq 2$, and $C_{2} \subset D$, with $K_{\widetilde{\mathcal{F}}} \cdot C_{2}=$ $-2+s\left(C_{2}, D\right)$. By the contractibility of $C_{2}, s\left(C_{2}, D\right) \geq 1$, i.e. $n \geq 3$, and we have a maximal sub-chain, $C_{2}, \ldots, C_{k}$ in $D$, with $k \geq 3$. As ever $K_{\widetilde{\mathcal{F}}} \cdot C_{k}=-1$, and we have a contradiction.

(b) $\Gamma$ is a maximal $-2 F$ chain, not meeting any $-1 F$ chain. This cannot happen since a maximal sub-chain in $D$ has both ends enjoying intersection -1 with $K_{\widetilde{\mathcal{F}}}$, or -2 if the length is 1 .

(c) The Dynkin diagram $D_{n}$, as described in III.3, including the degenerate case $D_{3}$. Here if $n \geq 4$, there is a maximal sub-chain in $D$ of the part of the diagram 
consisting of the $-2 F$ chain, which as in (b) cannot exist. So we're reduced to the degenerate case, and if $C$ is the curve with $K_{\widetilde{\mathcal{G}}} \cdot C=1$, then since $C^{2} \leq-2$, $D \cdot C=2$, so $\left(K_{\widetilde{\mathcal{F}}}+C\right) \cdot C=-1$. Whence again $C$ would have to be $\widetilde{\mathcal{F}}$ invariant, and find itself in the support of $D$, which cannot happen.

(d) An elliptic Gorenstein leaf. If the whole leaf isn't in $D$, then there is a nonempty maximal chain of $-2 F$ curves in both $T$ and $D$, which as above cannot exist, so indeed the whole leaf is also elliptic Gorenstein for $\widetilde{\mathcal{F}}$.

(e) A rational curve with a node is necessarily invariant for $\widetilde{\mathcal{F}}$ and $\widetilde{\mathcal{G}}$.

Whence in summary $\sigma$ can only contract $-1 F$ chains or e.g.l.'s, these are connected components of $D$ and everything contracted by $\sigma$ is contracted by $\rho$. This was of course under the hypothesis that $(\tilde{X}, \widetilde{\mathcal{G}})$ wasn't a rational fibration, however to prove that $T$ was in $D$ used no property of $\widetilde{\mathcal{F}}$, so conversely all $-1 F$ chains contracted by $\rho$ are $\widetilde{\mathcal{G}}$ invariant. Consequently $\widetilde{\mathcal{G}}$ is never a fibration since $\pi: \widetilde{X} \rightarrow B=\widetilde{X} / \widetilde{\mathcal{G}}$ exists in this case by the canonicity of the singularities and we can find fibres $\widetilde{X}_{b}$ disjoint from $D$, so everywhere transverse to $\widetilde{\mathcal{F}}$ from which the absurdity $K_{\widetilde{\mathcal{F}}} \cdot \widetilde{X}_{b}=\widetilde{X}_{b}^{2}=0$. A priori $\sigma$ is not unique since $\widetilde{\mathcal{G}}$ may have Kodaira dimension positive. Certainly we have $\nu(\widetilde{\mathcal{G}}) \geq 0$, and $\kappa(\widetilde{\mathcal{G}})=1$ is excluded by Bogomolov's generalisation of the Castelnuovo trick, i.e. if $h^{0}\left(N_{\widetilde{\mathcal{F}}}(D)^{\otimes m}\right) \geq 2$, $m \in \mathbb{N}$, we can take roots to show that $\widetilde{\mathcal{F}}$ is a fibration. Thus it remains to exclude, $\nu(\widetilde{\mathcal{G}})=\kappa(\widetilde{\mathcal{G}})=0$. Here we can contract the -1 chains for $\widetilde{\mathcal{G}}$, which are likewise for $\widetilde{\mathcal{F}}$ with the inverse ordering by, say, $\sigma^{\#}:(\widetilde{X}, \widetilde{\mathcal{G}}) \rightarrow\left(X^{\#}, \mathcal{G}^{\#}\right)$ so that $K_{\mathcal{G}}=N_{\mathcal{F} \#}\left(D^{\#}\right), D^{\#}=\sigma_{\#}(D)$, has canonical singularities for $\mathcal{F}^{\#}$ and $\mathcal{G}^{\#}$ and pass via an almost étale cover to obtain positive irregularity, which is absurd. Thus also for $\mathcal{G}, \kappa(\mathcal{G})=-\infty$, and $\nu(\mathcal{G})=1$, and whence $\rho=\sigma$ by the mutually unique contraction property with respect to either foliation. Whence finally we've got enough to apply $[\mathrm{TY}]$ or $[\mathrm{KoN}]$, where there is a more detailed treatment of the quotient singularities, i.e. for $\sigma^{\#}$ as above, and $D^{\#}=\sigma_{\#}(D)$ the elliptic Gorenstein leaves, necessarily in the non-singular locus of $X^{\#}$,

(a) $K_{X} \#+D^{\#}=K_{\mathcal{F} \#}+K_{\mathcal{G} \#}$ is nef, and $\left(K_{X \#}+D^{\#}\right) \cdot C=0$ for $C$ any curve iff $C \subset D^{\#}$.

(b) $\left(K_{X^{\#}}+D^{\#}\right)^{2}=2 K_{\mathcal{F} \#} \cdot K_{\mathcal{G} \#}=2 \rho^{*} K_{\mathcal{F}} \cdot K_{X}>0$, and $\rho^{*} K_{\mathcal{F}} \cdot\left(K_{X^{\#}}+D^{\#}\right)=$ $\rho^{*} K_{\mathcal{F}} \cdot K_{\tilde{X}}>0$ so $K_{X \#}+D^{\#}$ is big. 
Consequently $(\widetilde{X}, \widetilde{\mathcal{F}})$ is one of the naturally occurring foliations on a surface uniformised by the bi-disc, and the uniformisation map itself gives an almost étale cover $\nu:(S, \mathcal{H}) \rightarrow(X, \mathcal{F})$ such that the unique minimal desingularisation $(\widetilde{S}, \widetilde{\mathcal{H}}) \rightarrow(S, \mathcal{H})$ is obtained purely by resolving cusps.

While a large part of this discussion has involved the analytic topology, it has, nevertheless been motivated by purely algebraic conceptions, i.e. Kodaira dimensions and abundance, and from this point of view let us summarise IV.4-5 by way of,

Theorem 3 IV.5.11. Let $(X, \mathcal{F})$ be the canonical model of a foliation of numerical Kodaira dimension 1 then either,

(a) Abundance holds, and the Kodaira fibration co-incides with the foliation, which is thus a non-isotrivial elliptic fibration, IV.4.4. (i).

(b) Abundance holds, but the Kodaira fibration is distinct from the foliation, so it is either Riccatti, IV.4.4.(ii), or turbulent, IV.4.4.(iii), or an isotrivial family of hyperbolic curves, IV.4.4.(iv).

(c) Abundance fails, so the Kodaira dimension is $-\infty$, IV.5.1, and the canonical model is either of the natural foliations on the Baily-Borel compactification of a Hilbert-Modular surface.

All of which, we've proved, except to note that by, $[\mathrm{M}]$, a bi-disc quotient necessarily arises from a real quadratic arithmetic structure, and the identity of the compactification with the canonical model follows from IV.2.2 and I.2.4.(a). The reader may also have observed that the current section could have been simplified a little by the systematic use of algebraic stacks, since,

Remark IV.5.12 Technically, IV.5.1.bis is somewhat simpler using algebraic stacks, especially from the point of view of the stack interpretation of IV.5.4, although the Riemann-Roch formula is more complicated. The rest of IV.5.1 is, however, a good bit easier since the intervention of symbolic powers of ideals, as opposed to powers, is precisely a result of the difference between a stack and its moduli, while IV.5.9-10 are a one line exercise in the 2-category of stacks. Such simplifications are, however, technical rather than essential, whereas the 
use of the Gorenstein covering stack in the definition of the Poincaré metric is a conceptual necessity.

\section{Conformal structure}

\section{V.1. Uniform uniformisation}

The basic question is to what extent the equivalence of classification by Kodaira dimension with the elliptic, parabolic, hyperbolic trichotomy in the uniformisation theory of algebraic curves continues to hold for foliations by curves. As such the key objects are,

Definition V.1.1. Given a complete foliated surface $(X, \mathcal{F})$ or algebraic stack we denote by $\operatorname{Hom}_{\mathcal{F}}(\Delta, X)$ the space of invariant maps from discs in the compact open topology, and by $\overline{\operatorname{Hom}}_{\mathcal{F}}(\Delta, X)$ the space of invariant discs with invariant bubbles where the compact open topology is extended by way of the Hausdorff metric on graphs. In particular for $\mathcal{X} \rightarrow X$ the Gorenstein covering stack (by slightly abusive definition identical with $X$ at any egl's) of the canonical or even just minimal model of a foliated surface,

$$
\widetilde{\operatorname{Hom}}_{\mathcal{F}}(\Delta, X)=\overline{\operatorname{Hom}_{\mathcal{F}}(\Delta, \mathcal{X} \backslash \operatorname{sing}(\mathcal{F}))},
$$

where the closure is taken in $\overline{\operatorname{Hom}}_{\mathcal{F}}(\Delta, \mathcal{X})$. Furthermore for $B$ a proper invariant sub-variety or stack we say,

(a) $(X, \mathcal{F})$ is hyperbolic modulo $B$ if a sequence $\left(f_{n}\right)$ in $\overline{\operatorname{Hom}}_{\mathcal{F}}(\Delta, X)$ without a convergent sub-sequence is arbitrarily close to $B$, i.e. the image of every compact is arbitrarily close to $B$.

(b) The leaves of $(X, \mathcal{F})$ are hyperbolic modulo $B$ if a sequence $\left(f_{n}\right)$ in $\widetilde{\operatorname{Hom}}_{\mathcal{F}}$ $(\Delta, X)$ without a convergent sub-sequence is arbitrarily close to $B$.

As it happens, there are several subtleties about the use of the word invariant should we wish to extend these definitions to dimension greater than 2, cf. [M7] VI.4. A further subtlety that might occur without the invariance condition is how one extends these definitions to a quasi-projective situation, where say $X$ is compactified by way of a boundary $D$. In such circumstances, already in dimension 
2 , one must allow in a non-foliated definition of hyperbolic the possibility that sequences of discs become arbitrarily close to $D$ unless $D$ is a stable curve. With the invariance condition, however, there is no practical need to consider anything other than non-invariant boundaries, so to all intents and purposes (a) and (b) may be naively extended to the quasi-projective setting. As such the only important point is the difference between (a) and (b) themselves, or better between $\overline{\text { Hom }}$ and $\widetilde{\text { Hom }}$. Again, the motivation is the same as that for the Poincaré metric, i.e. the capacity of 'leaves' should have good lower semi-continuity properties. Better still,

Fact V.1.2 (cf. [Br5] Lemma 0) Suppose that $(X, \mathcal{F})$ is a canonical or minimal model of a foliated surface with $\mathcal{X} \rightarrow X$ its Gorenstein covering stack then,

$$
\widetilde{\operatorname{Hom}}_{\mathcal{F}}(\Delta, X)=\operatorname{Hom}_{\mathcal{F}}(\Delta, \mathcal{X} \backslash \operatorname{sing}(\mathcal{F})),
$$

i.e. if a sequence of discs in the latter space converges to a disc with bubbles then it converges to an honest disc.

Proof The idea of op. cit. is that given a sequence $\left(f_{n}\right)$ converging to a disc with bubbles $f$ ones seeks a holomorphic interpolation of it, i.e. a holomorphic family $f_{t}$ converging to $f$ or what amounts to the same thing a meromorphic map from the bi-disc to $X$ which sends non-special fibres over say the first projection to invariant curves missing the singularities. A foliated version of the bend and break lemma then implies that either this map is actually holomorphic everywhere or there are $K_{\mathcal{F}}$ negative rational curves. There are, however, some issues about the existence of such a holomorphic interpolation, cf. [CI], albeit that these may be comfortably taken care of by way of the principle facts about hyperbolicity which will follow.

In this context, it is most convenient to work directly on a canonical model $(X, \mathcal{F})$ of a foliated surface, and to introduce the necessarily proper algebraic sets,

(a) $B$ of invariant algebraic curves meeting at least one singularity, and,

(b) $P$ the parabolic part of $B$, i.e. the irreducible components of $B$ whose complement with $\operatorname{sing}(\mathcal{F})$ are parabolic Riemann-surfaces, or more generally, parabolic stacks. 
There is in fact quite an interesting relation between neighbourhoods of components of $P$ on foliated surfaces of general type and non-hyperbolic Riccatti foliations. Let us, however, for the moment observe that a component of $P$ must either have non-negative square, or pass through a saddle node, so, for example, on a foliated surface of general type without saddle nodes $P$ is empty, which should give a little feeling for,

Fact V.1.3. If the (positive) cone generated by $K_{\mathcal{F}}$ and $K_{X}$ contains a big divisor then, $(X, \mathcal{F})$ is hyperbolic modulo $B$ and the leaves are hyperbolic modulo $P$.

The related statement of the non-Zariski denseness of invariant entire mappings to such foliated surfaces is the main theorem of [M1]. Slightly more precisely the main theorem of op. cit. is only stated for $X$ of general type, but this is only used to guarantee $K_{\mathcal{F}} \cdot K_{X}>0$ if $K_{\mathcal{F}}^{2}=0$. The (non-evident) refinement from op. cit. to V.1.3 is in [M4] V.5.1, albeit that the essential of the method remains the same, while $[\mathrm{DV}]$ affords some simplification.

Now, needless to say, the corollary of V.1.3 that the Poincaré metric is continuous should $K_{\mathcal{F}}$ be nef. with Kodaira dimension $-\infty$ has already been employed in a critical way in the completion of the classification theorem from IV.5.8 on, which in turn only involves the weaker statement on the hyperbolicity of the leaves, and it is this type of hyperbolicity that ties in well with classification. Indeed,

Corollary V.1.4. If the leaves of $(X, \mathcal{F})$ are not hyperbolic modulo $P$ then they are uniformly parabolic and either,

(a) The foliation has a first integral, and is a fibration in rational or elliptic curves.

(b) There is an étale covering of the Gorenstein covering stack on which the foliation is given by a global vector field.

(c) A Riccatti, IV.4.4(ii), or turbulent, IV.4.4(iii) foliation arising from a representation of the fundamental group of an orbifold with non-negative Euler characteristic.

Plainly, there is effectively nothing here to prove, which isn't covered by V.1.3 and 
the classification theorem. What we do observe, however, is that the classification by Kodaira dimension, and the classification by the ellipticity, parabolicity, or hyperbolicity of the leaves are not quite the same, and, in fact suggests a refinement in the former which merits a new section.

\section{V.2 Bi-rational vs. Bi-analytic}

Quite generally an analytic map between complete normal varieties defined in co-dimension 1 is actually defined in co-dimension 2 and meromorphic as soon as it is meromorphic in co-dimension 1. It may, however, be that there are some essential singularities in co-dimension 1, which suggests we make,

Definition V.2.1. A pair of canonical models $\left(X_{i}, \mathcal{F}_{i}\right)$ of foliated surfaces, or algebraic stacks, $i=1$ or 2 , are said to be bi-analytic if there are invariant divisors $D_{i}$ in $X_{i}$ and an analytic isomorphism,

$$
\left(X_{1} \backslash D_{1}, \mathcal{F}_{1}\right) \simeq\left(X_{2} \backslash D_{2}, \mathcal{F}_{2}\right),
$$

respecting the foliations.

Now, the very definition of hyperbolicity of the leaves, or more pertinently their parabolicity, is a property of the Gorenstein covering stack, and whence is wholly independent of any étale covering of the same as found in IV.3.6 or IV.4.4.(iii). Nor does it depend on coverings ramified in parabolic leaves. With this simplification in mind, we can eschew some of the complication in IV.4.4(ii), to assert that the foliations occurring in V.1.4(c) are bi-analytic to those defined by global vector fields. Indeed in IV.3.6 there occur 6 types of group varieties, of which 4 are non-compact. Amongst the 4 non-compact types, there are 2 where the group variety structure is necessarily iso-trivial, viz: $\mathbb{G}_{a} \times \mathbb{G}_{m}$, and $\mathbb{G}_{m} \times \mathbb{G}_{m}$, and the foliations of IV.3.6 (including only log-canonical ones on the latter) arising from global vector fields may also be bi-analytically compactified by the procedure of IV.4.8 (with at least one singularity as per IV.4.8.iii) to Riccatti foliations of Kodaira dimension 1. The remaining 2 types of group varieties, i.e. semi-abelian surfaces or vectorial extensions of an elliptic curve, can only be compactified, by the analogous procedure IV.4.11, to turbulent foliations of Kodaira dimension 1 when the group structure is iso-trivial, i.e. we have $E \times \mathbb{G}_{a}$ or $E \times \mathbb{G}_{m}$ for $E$ an elliptic curve. As such, 
Fact V.2.2. Up to possibly ramified covering in the boundary the uniformly parabolic foliations V.1.4(c) are bi-analytic to foliations defined by vector fields on $\mathbb{G}_{a} \times \mathbb{G}_{m}$, or $\mathbb{G}_{m} \times \mathbb{G}_{m}$ (Riccatti case), respectively $\mathbb{G}_{a} \times E$, or $\mathbb{G}_{m} \times E$ (turbulent case, $E$ elliptic) which admit bi-rational extensions to global vector fields as found in IV.3.6. The various open sets where the bi-analytic isomorphism is defined are $\mathbb{G}_{a} \times \mathbb{P}^{1}, \mathbb{G}_{m} \times \mathbb{P}^{1}, \mathbb{G}_{a} \times E$, and $\mathbb{G}_{m} \times E$ respectively

The extremely elegant, and satisfactory, identification of foliations with parabolic leaves as found in V.1.4 and V.2.2 should not, however, obscure,

Warning V.2.3. The properties of a foliated variety being hyperbolic and having hyperbolic leaves are not synonymous. In particular there exist foliations $(X, \mathcal{F})$ with hyperbolic leaves modulo $P$, but, which nevertheless admit invariant entire curves $f: \mathbb{C} \rightarrow X$ with Zariski dense image. Indeed, consider a generic representation $r$ of $\mathbb{Z}^{2}$, identified with $H_{1}\left(\mathbb{P}^{1} \backslash\{0,1, \infty\}\right)$, in $\mathrm{PGL}_{2}$. For a compactification without saddle nodes there will be precisely 2 parabolic invariant curves not contracted by the Kodaira fibration. These curves are isomorphic to $\mathbb{P}^{1}$, so, necessarily algebraic. If, however, we compactify over infinity as in IV.4.8.iii by saddle nodes such that the weak branches do not converge, which, cf. IV.4.9, we can certainly do, we will obtain a foliation on a projective surface whose complement by the fibre at infinity is bi-analytic with the saddle node free compactification complemented by it's fibre at infinity, and whence two parabolic curves. Since, however, there are no convergent weak branches at infinity neither of these curves can be algebraic. As such, beyond the obvious, i.e. the image of the Kodaira fibration cannot be a hyperbolic curve, the classification of hyperbolic Riccatti and to a lesser extent turbulent foliations is a lot more delicate than V.1.4, albeit that anything of positive numerical Kodaira dimension which isn't hyperbolic modulo the algebraic curves through the singularities must be Riccatti or turbulent.

Irrespectively hyperbolic or parabolic leaves is a good property from a classification point of view, and we may further observe that the foliations with hyperbolic leaves modulo $P$ and not of general type admit an extremely uniform uniformisation. Indeed for cases of positive Kodaira dimension there is a short exact sequence,

$$
1 \longrightarrow K \longrightarrow \Gamma \longrightarrow \operatorname{Aut}(F)
$$


with $\Gamma$ Fuchsian of the first kind, $F$ an algebraic curve, and $K$ contains no elliptic elements. This gives us a Riemann surface $S$ on quotienting the disc by $K$, and an identification of the foliation with a compactification of the map of classifying stacks,

$$
[S \times F / \Gamma] \longrightarrow\left[F / \Gamma_{0}\right]
$$

where $\Gamma_{0}$ is the image of $\Gamma$. Should only the numerical dimension be positive then $F$ can be a disc rather than an algebraic curve, and $\Gamma$ while a group of automorphisms of the disc need not be Fuchsian. Nevertheless a quotient of the corresponding bi-disc is still quasi-projective, albeit that the compactification as a canonical model is unique. In either case, however, what distinguishes these foliations with hyperbolic leaves from the general type case is,

Fact V.2.4. If $(X, \mathcal{F})$ is not of general type but has hyperbolic leaves modulo $P$ (where $P$ includes, slightly abusively the contraction of any egl's) then there is an étale covering map $\Delta \times F$, where $F$ is a Riemann surface (in fact an algebraic curve or a disc) to $X \backslash P$, with the foliation being simply the projection to $F$.

Alternatively, if one prefers, there is 'no modular variation of the leaves', which merits further elaboration by way of,

\section{V.3 Modular Variation}

If we return to the notations of $\S \mathrm{IV} .5$, and in particular the Bochner type formula pre-ceding IV.5.7 with $\varphi$ the Poincaré metric, then an expansion in local foliation coordinates reveals that,

$$
\int_{X} e^{-2 \varphi} \nabla c_{\mathcal{F}}\left(\bar{K}_{\mathcal{F}}\right) \wedge \overline{\nabla c_{\mathcal{F}}\left(\bar{K}_{\mathcal{F}}\right)}
$$

up to some irritating normalising constant is a measure of the variation from leaf to leaf of the conformal structure defined by the Poincaré metric. Indeed a varying family of metrics on a 1-dimensional family of Riemann surfaces determines not just a tangent direction but a tangent vector in the moduli, and if we were in a situation where the moduli stack $[X / \mathcal{F}]$ admitted a coarse moduli space $X / \mathcal{F}$ then the above expression would be nothing other than the squared $\ell_{2}$ norm of this tangent vector in the Weil-Petersson metric. Evidently, such a precise identification is not possible for an arbitrary foliation, and this misfortune 
remains true even in the presence of the Fubini theorem of [M6]. Nevertheless the discussion of IV.5 not only confirms that this expression continues to have sense even if $\varphi$ is no better than continuous (nor degenerates too rapidly at the singularities and/or parabolic leaves, which it doesn't, cf. [M4] V.5.1(b)) but, moreover, if this expression were too vanish then we would indeed find ourselves in the situation of V.2.4, i.e. with nil variation of the conformal structure. Better still, the said Bochner formula continues to hold even for foliations of general type, so that while no longer enjoying a pointwise sense, it is, nevertheless true, that the value of the aforesaid " $\ell_{2}$-norm" is, again up to some irritating constant, $K_{\mathcal{F}}^{2}$.

More generally, we could consider a conformal structure/metric (with singularities) $e^{\psi}$ on $K_{\mathcal{F}}$ arising from the restriction to $K_{\mathcal{F}} \otimes \overline{K_{\mathcal{F}}}$ of the curvature form of a positively metricised bundle $\bar{L}$, in fact positive in the leaf direction would be sufficient, and as a measure of its variation,

$$
\int_{X} e^{-2 \varphi} \nabla c_{\mathcal{F}}(\bar{L}) \wedge \overline{\nabla c_{\mathcal{F}}(\bar{L})}
$$

but in these circumstances the Bochner formula reveals rather less, so that, up to a normalising constant this is equal to,

$$
\int_{X} c_{1}\left(K_{\mathcal{F}}\right) c_{1}(L)+\frac{1}{2} K(\psi) c_{1}(\bar{L})^{2}
$$

where the curvature $K(\psi)$ of $e^{\psi}$ along the leaves is normalised in such a way as to be -1 on the Poincaré metric. As such the transparency that the Poincaré metric brings to the discussion, and in particular, the interpretation of the invariant $K_{\mathcal{F}}^{2}$ on the canonical model should be evident.

All of which is more or less true independently of whether one knows that the variation of the Poincaré metric is psh. In fact what threatens to fail is above all the definability of our " $\ell_{2}$-norm", and the fact that it really is a norm rather than a pseudo-norm. Nevertheless, the said pluri-subharmonic variation, or more correctly aspects of its demonstration sheds yet more light on the question of modular variation by way of the proof of,

Fact V.3.1 Let $f: X \rightarrow \Delta$ be a pseudo-convex family of Riemann surfaces over the disc with $S$ the image of a section $\sigma$, i.e. $f$ is proper or there is a psh function $\psi: X \rightarrow[\infty, \infty)$ exhaustive in the fibre direction, then the logarithmic capacity of the fibres $X_{b}, b \in \Delta$ seen from $\sigma(b)$ has pluri-sub harmonic variation along $S$. 
Proof (cf. [Y]) Fix a local equation, $s$, say, for $S$, and $R<\infty$, so that for $b$ there is a Green's function $g_{R, b}$, necessarily admitting an expansion about $\sigma(b)$ of the form,

$$
g_{R, b}=\log |s|_{X_{b}} \mid-\lambda_{R . b}+0\left(|s|_{X_{b}} \mid\right)
$$

To avoid some pointless, and not in the least illuminating, consideration we will further suppose that on considering the function,

$$
g_{R}: X(R):=\{x: \psi(x)<R\} \rightarrow[-\infty, 0]: x \mapsto g_{R, f(x)}
$$

we continue to have such an asymptotic expansion in a neighbourhood of $S$, i.e. we can just drop the suffix $b$ in the original expansion. Otherwise there is need for some regularisation argument akin to those in IV.5. Supposing, again for the sake of convenience rather than necessity, sufficient regularity of $g_{R}$ everywhere, we see, that understood naively,

$$
\left(d d^{c} g_{R}\right)^{2} \leq 0
$$

Where, understood naively means take the absolutely continuous part. Shrinking $\Delta$ as necessary, and supposing $R$ sufficiently large, we apply, following [Y], Stoke's theorem to the integral,

$$
\int_{0}^{1} \frac{d t}{t} \int_{X(R) \cap f^{-1}(\Delta(t))}\left(d d^{c} g_{R}\right)^{2}
$$

where $\Delta(t)$ is the disc of radius $t$. This reveals 3 terms. The first is, up to a minus sign, what we're looking for, i.e.

$$
\int_{0}^{1} \frac{d t}{t} \int_{S(t)} d d^{c} \lambda_{R}
$$

The second admits a further application of Stokes, and comes out as,

$$
\int_{\partial \Delta} g_{R} d d^{c}\left(g_{R}\right) d^{c} \log |b|
$$

for $b$ the standard coordinate on $\Delta$. Since in this formula only the absolutely continuous part of $d d^{c}\left(g_{R}\right)$ should be understood, this is necessarily zero by the harmonicity of the Green's function in the $f$ direction, and off $S$. Consequently, we have the ultimate term,

$$
\int_{0}^{1} \frac{d t}{t} \int_{\partial X(R) \cap f^{-1}(\Delta(t))} d^{c} g_{R} d d^{c} g_{R}
$$


and it's here that we need the pseudo convexity. Indeed, again supposing sufficient smoothness for convenience, on $\partial X(R) \cap f^{-1}(\Delta(t)), d^{c} \psi d d^{c} \psi$ and $d^{c} g_{R} d d^{c} g_{R}$ have the same sign, which by pseudo-convexity, is non-negative. Whence, putting everything together,

$$
\int_{0}^{1} \frac{d t}{t} \int_{S(t)} d d^{c} \lambda_{R} \leq 0
$$

and letting $R$ go to infinity we conclude.

The above discussion is nothing other than the proof of the main theorem of [Y] re-written without an irksome choice of a square root of -1 and local coordinates. Nevertheless such a re-writing reveals many things such as: op. cit.'s condition of a Levi-flat, as opposed to pseudo-convex, boundary being over kill, the topology of the fibres is un-important, and for that matter that the entire discussion generalises perfectly to Monge-Ampère capacities. More pertinently, however, we see that a lack of variation of the logarithmic capacity along the section $S$ is extremely rare. Supposing that all the fibres of $f$ are hyperbolic then it almost forces the boundary to be Levi flat, and more surprisingly the vanishing of $\left(d d^{c} g\right)^{2}$, for $g$ the limit of the $g_{R}$ 's. Consequently, in such circumstances the holomorphic 1-forms $\partial g_{b}$ do not admit any variation in $b$, which, should all the fibres of $f$ be simply connected, effectively amounts to the existence of a holomorphic trivialisation of $f: X \rightarrow \Delta$ as a projection $\Delta \times \Delta \rightarrow \Delta$ from a bi-disc.

To see the relevance of this discussion to foliations by curves one has to introduce a couple of groupoids defined over the locus $\mathcal{U}$ where the Gorenstein covering stack of a canonical model of a foliation $(X, \mathcal{F})$ is smoothly foliated. In the first place there is the usual holonomy groupoid, $\mathcal{F} \rightrightarrows \mathcal{U}$ which, if one is worried about set theory problems, is easily checked to be a representable groupoid in analytic stacks, [M5] A.1. One then replaces the orbits by their fibre by fibre homotopy cover, and this defines a groupoid with the same orbits, but potentially radically different stabilisers, i.e. the homotopy groupoid, cf. op. cit. A.2, $\mathcal{H} \rightrightarrows \mathcal{U}$ This gives a representable fibration of analytic stacks $f: \mathcal{U} \rightarrow[\mathcal{U} / \mathcal{H}]$, so that if we take an étale transversal $T \rightarrow[\mathcal{U} / \mathcal{H}]$ then we obtain by base change a family of simply connected Riemann surfaces,

$$
f_{T}: \mathcal{U} \times_{[\mathcal{U} / \mathcal{H}]} T \rightarrow T
$$

and it's to this family that we can apply our considerations and reflections on the 
proof of V.3.1. As it happens, and viewed from this perspective, perhaps necessarily, $f_{T}$ is defined (in a more elementary way) and the study of it's convexity is the principal object (where it's called a covering tube) of [Br5]. As such, what the introduction of analytic stacks principally achieves is philosophical clarity, i.e. it really is true,

Fact V.3.2 If $f: \mathcal{U} \rightarrow[\mathcal{U} / \mathcal{H}]$ is the fibration in analytic stacks of the (small) homotopy groupoid of the canonical model of a foliated surface $(X, \mathcal{F})$ then $(X, \mathcal{F})$ has general type iff its leaves are hyperbolic modulo a proper algebraic set, and, there is non-nil variation of the conformal structure of the hyperbolic leaves.

This is simply a re-capitulation of the above discussion. Regrettably we are un-able to make any assertion about the extent of the variation, e.g. it would be extreme optimism (but rather pleasant) if the integrand in our measure of the conformal variation were non-zero off a proper algebraic set. Necessarily, however, it must be non-zero on a set of positive Lebesgue measure, since the integrand is really defined and absolutely continuous once one knows that the Poincaré metric is continuous.

\section{V.4 Hyperbolicity and abundance}

A revealing feature of the previous discussion V.3 is the naturality of the discussion IV.5. As such, it's very much true that not only the classification theorem, but also the identification of foliations of general type with varying conformal structure flows from the hyperbolicity theorem V.1.3. Granted, there are some complications, such as the regularisation arguments of IV.5, or Brunella's lemma, [Br5], that the conditions of V.3.1 are verified, but these latter phenomenon are no more difficult in dimension 2 than in arbitrary dimension. Of course in principle, V.1.3 shouldn't be specific to dimension 2 either. Since, however, it plays an even more central role, indeed effectively implies classification and more, than abundance in classical Enriques-Kodaira theory, it is to be expected that the difficulty of proving it grows as the ambient dimension increases, even though the leaf dimension remains fixed. Consequently, one can derive further feeling for V.1.3, and exactly how it replaces abundance by a brief over view of the proof.

Naturally to this end, let's suppose that V.1.3 were false. As such we sup- 
pose that there is a sequence of discs $\left(f_{n}\right)$ divergent in $\widetilde{\operatorname{Hom}}_{\mathcal{F}}(\Delta, X)$, which, in the compact open sense, are not arbitrarily close to the divisors $P$, or $B$ as appropriate. Now to this data it is a quite general fact, i.e. independent of any foliation hypothesis, cf. [M2], that we can associate a closed positive current $T$. Basically, this current is the weak limit of (a subsequence) defined by integration over a compact in the $n$th disc normalised by its (necessarily unbounded in $n$ ) area. More strictly speaking we use a further averaging of the area as found in Nevanlinna theory, cf. op. cit., which for a suitable choice of origin of each disc allows us to deduce the extra information that $C . T \geq 0$ for every curve $C$ supported on $P$, or $B$ as appropriate. Again, this is a general phenomenon, and an equally general phenomenon is that we can similarly define a derivative $T^{\prime}$, which is a closed positive current in $\mathbb{P}\left(\Omega_{X}^{1}\right)$ (or better $\mathbb{P}\left(\Omega_{\mathcal{X}}^{1}\right)$, for $\mathcal{X}$ the minimal smooth stack structure on a resolution of any elliptic singularities on the canonical model) which pushes forward to $T$. In particular for $L$ the tautological line bundle, we have in full generality,

V.4.1 Fact ([M2]) (The tautological inequality) $L . T^{\prime} \leq 0$

In a certain sense this could even be taken as a definition of $L$. Alternatively, one could think (and indeed one can) repeat the above process with a sequence of maps $f_{n}: C_{n} \rightarrow X$ from smooth compact curves of genus $g_{n}$ of unbounded degree, in which case, after subsequencing, the analogue of $T$ is,

$$
\lim _{n \rightarrow \infty} \frac{1}{H \cdot f_{n} C_{n}} \int_{C_{n}}\left(f_{n}\right)^{*}
$$

while for $T^{\prime}$ we replace $f_{n}$ by it's derivative $f_{n}^{\prime}$, so that,

$$
L . T^{\prime} \leq \lim _{n \rightarrow \infty} \frac{\left(2 g_{n}-2\right)}{H \cdot f_{n} C_{n}}
$$

The role of the quantity on the right for sequences of discs is to ask that the discs become more and more parabolic, so for the algebraic version, closer and closer to rational or elliptic, i.e. the right hand side goes to zero. All of which is a bit of a tautology since the left hand side is, manifestly, the average of the (negative) euler characteristic for our sequence.

More specifically to our foliation situation, however, we observe that the $f_{n}^{\prime}$, 
must factor through,

$$
\widetilde{X}=\operatorname{Bl}_{Z}(X)=\operatorname{Proj}\left(\sum_{n=1}^{\infty} K_{\mathcal{F}}^{\otimes n} I_{Z}^{n}\right) \hookrightarrow \mathbb{P}\left(\Omega_{X}^{1}\right)
$$

where the quotient $\Omega_{X}^{1} \rightarrow K_{\mathcal{F}} I_{Z} \rightarrow 0$, with $Z$ the singular locus of the foliation, defines the very foliation itself, as in II.3.1. Consequently, if as per op. cit., $E$ is the exceptional divisor then $T^{\prime}$ is a closed positive current on $\tilde{X}$, and V.4.1 becomes,

$$
K_{\mathcal{F} . T} \leq E . T^{\prime}
$$

. All of which is very much true for an arbitrary foliation by curves, and what is crucial is,

Fact V.4.2 ([M4] V.4.4) (Refined Tautology) Hypothesis as above on the $\left(f_{n}\right)$, i.e. they are not arbitrarily close to $P$ or $B$ as appropriate, then provided the singularities of $(X, \mathcal{F})$ are canonical,

$$
K_{\mathcal{F}} \cdot T \leq 0
$$

Despite appearances, it is critical to understand that V.4.1 and V.4.2 are very different. The best way to see this is not to suppose that the discs diverge. As such, V.4.1 may be formulated as a tautological identity, cf. [M2], with an appropriate negative euler characteristic on the right, and has nothing to do with foliations, i.e. it becomes the very definition of holomorphic sectional curvature in integrated form. Whence, what V.4.2 says, in its stronger form as a refined identity, is that in the presence of canonical singularities (and if we're considering all invariant discs only in the presence of canonical singularities) $K_{\mathcal{F}}$ measures the curvature along the leaves. A perfectly good example of this already occurs for algebraic Gorenstein curves $C$ considered as foliations $C \rightarrow$ pt. Manifestly, there is no difficulty in constructing examples such that $\omega_{C}$ (i.e. $K_{\mathcal{F}}$ for this example) is ample, which if $\omega_{C}$ truly reflected the curvature ought to imply that $C$ is negatively curved, but we may make examples whereby $C$ is so singular that it is actually rational, i.e. anything but negatively curved.

With this important caveat in mind, let us review the principle why canonical singularities yield V.4.2, leaving the details to op. cit. As such, consider the 
typical picture of a canonical singularity,

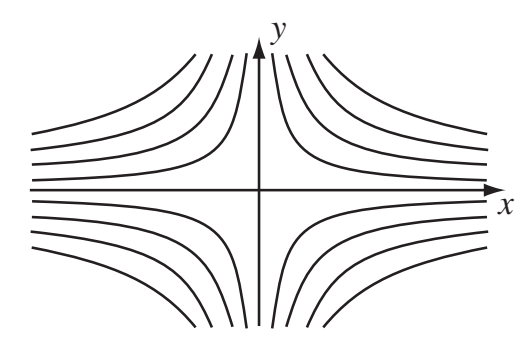

and let us put ourselves in the situation that our invariant discs are not arbitrarily close to $B$. We require to bound $E . T^{\prime}$ by 0 as defined prior to V.4.2. Morally (though not quite precisely) such an intersection can only occur if our discs happen to pass along the $x$ or $y$ axis in our picture. Therefore $p$ times the intersection is not governed by an intersection with the ideal $(x, y)^{p}$ (or more correctly its blow up) but $\left(x, y^{p}\right)\left(x^{p}, y\right)$ whose co-dimension in the local ring at the origin grows like $p$ as opposed to $p^{2}$. Furthermore, up to some irrelevant constants, for $H$ ample,

$$
\Gamma\left(X, H^{\sqrt{p}}\left(x, y^{p}\right)\left(y^{p}, x\right)\right)
$$

has lots of sections, so if our discs avoid at least one of these,

$$
E . T^{\prime} \leq \frac{1}{\sqrt{p}} H \cdot T
$$

which plainly goes to zero as $p$ goes to infinity. With a little further work, one shows that for $p$ sufficiently large, the linear systems in question have base locus $B$, while a slightly more subtle calculation proper to the case of hyperbolic leaves reduces the problem to $P$ under this more restrictive hypothesis.

This sketch of the proof of V.4.2 indicates why the theorem is not asserted 
for a log-canonical singularity,

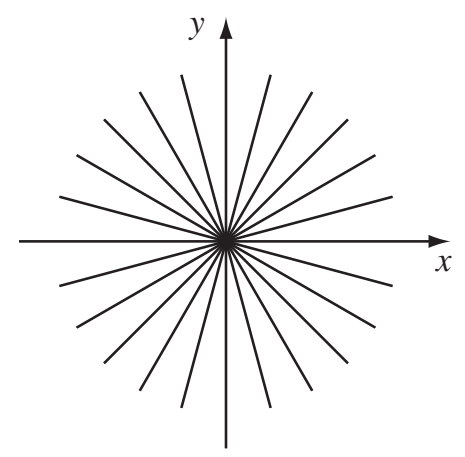

since, plainly, the conditions of vanishing along the foliation to order $p$ at the origin, and vanishing to order $p$ at the origin are no different. Nevertheless, it isn't simply a question that the proof doesn't work, but there is, in fact, absolutely no difficulty in constructing counterexamples to V.4.2 for such a singularity.

Now, as it happens, all of the above discussion continues to have sense in a context much wider than foliations by curves, which we may think of as a fibration $X \rightarrow[X \mathcal{F}]$ of $X$ over its classifying stack. Indeed there is an analogy of all of the above for a variety $X$ considered as foliated/fibred by way of a map $X \rightarrow \mathrm{pt}$ from $X$ to a point. Thus, while the details have only been checked in these two cases, one could reasonably imagine that all of the above holds in some massive generality such as possibly not even representable maps $f: \mathcal{Y} \rightarrow \mathcal{S}$ of analytic stacks with relatively canonical singularities. As such what is specific to the situation of foliation by curves is not V.4.1 or even V.4.2 (functorially understood) but,

Fact V.4.3 Our closed positive current $T$ is, in fact, a transverse invariant measure. Indeed, functorially understood, there is a measure $d \mu_{[X / \mathcal{F}]}$ on the classifying stack $[X / \mathcal{F}]$ whose pull-back is $T$.

According to a classical definition of transverse invariant measure, V.4.3 is immediate. A better definition, [M6], in terms of Borel functions with compact support as a co-Cartesian functor would take us rather too far out of our way. Whence we'll simply reference the latter part of V.4.3 to op. cit., although it may be useful to note that op. cit. permits one to re-interpret $d \mu_{[X / \mathcal{F}]}$ as a Radon measure on a certain space, and even to integrate along the foliation, so that 
we obtain a rather instructive application of a wholly general Fubini theorem whereby,

$$
K_{\mathcal{F} . T}=\int_{[X / \mathcal{F}]} d \mu_{[X / \mathcal{F}]} \int_{\mathcal{F}} c_{1}\left(\bar{K}_{\mathcal{F}}\right)
$$

where we view a metricisation $\bar{K}_{\mathcal{F}}$ of $K_{\mathcal{F}}$ as a family of conformal structures parametrised by $[X / \mathcal{F}]$. Which, again, should hopefully clarify the difference between V.4.1 and V.4.2.

Irrespectively, if we further put ourselves in the situation where $(X, \mathcal{F})$ is a canonical or even just minimal model, then by V.4.2 we must actually have $K_{\mathcal{F}} . T=0$. Furthermore in the situation of V.1.3 this is impossible if $K_{\mathcal{F}}^{2}>0$, e.g. apply IV.2.1, so in fact we find ourselves immediately reduced to the hypothesis required for classification, i.e. $K_{\mathcal{F}} \cdot K_{X}>0$, and $K_{\mathcal{F}}^{2}=0$, cf. IV.5.8. As it happens, there is no harm in making the wholly general hypothesis that the discs $f_{n}$ are not arbitrarily close to any divisor so $T$ is nef., although it actually happens that this follows from V.4.3 and the definition of $B$. Irrespectively, the Hodge index theorem yields,

Fact V.4.4. Suppose V.1.3 is false, then there is a canonical model of a foliation $(X, \mathcal{F})$ satisfying exactly the hypothesis IV.5.8 critical to classification, i.e. $K_{\mathcal{F}}^{2}=$ 0 and $K_{\mathcal{F}} \cdot K_{X}>0$, such that in co-homology $K_{\mathcal{F}}$ is parallel to a transverse invariant measure $d \mu_{[X / \mathcal{F}]}$.

The measure in question is of course $T$, but from now on we'll employ the measure notation to emphasise exactly what's going on, i.e.

Rephrasing V.4.5. If a situation whereby the rather general considerations of V.3 (as detailed towards the end of IV.5) were not to yield that foliations with $\nu(\mathcal{F})=1$ and $\kappa(\mathcal{F})=-\infty$ are extremely special then $K_{\mathcal{F}}$ (which we cannot hope to represent by a divisor) would be represented not by any old positive current, but, in fact, a transverse invariant measure.

As it happens, in arbitrary dimension $m$, one finds, under conditions analogous to V.1.3, that $K_{\mathcal{F}}^{m-1}$ is represented by a transverse invariant measure, by way of a higher dimensional index theorem, cf. [M7] II.2. More, immediately, however, let us see why this representative is special. Observe that where the topus of $[X / \mathcal{F}]$ is étale, $K_{[X / \mathcal{F}]}$ is well defined, and a line bundle on $[X / \mathcal{F}]$, and 
since this holds away from the singularities of $\mathcal{F}$ and $X$ is normal, it extends to a line bundle on $X$ satisfying,

$$
K_{X}=K_{\mathcal{F}}+K_{[X / \mathcal{F}]}
$$

Applying V.4.4, we therefore deduce that under the hypothesis of the same,

$$
K_{[X / \mathcal{F}]} \cdot d \mu_{[X / \mathcal{F}]}>0
$$

Now morally, this is impossible since $d \mu_{[X / \mathcal{F}]}$ is a class in the top dimension on $[X / \mathcal{F}]$ while $K_{[X / \mathcal{F}]}$ is a class in (real) co-dimension 2 . The only flaw in this argument results at points where the topus of $[X / \mathcal{F}]$ is not étale, i.e. at the singularities, where it may be no better than smooth, and around which $K_{[X / \mathcal{F}]}$ fails to satisfy a descent datum. As it happens a similar objection on some substantially bigger site (the essentially quasi finite site, cf. [M6]) could be posed in respect of whether $d \mu_{[X / \mathcal{F}]}$ is really a measure on $[X / \mathcal{F}]$ or just the étale part. In this latter case, however, we can talk about Radon measures on the étale part of finite mass, so there is, in fact, a well defined extension by zero across the singularities. Regardless, the conclusion of this discussion is,

Fact V.4.6 The intersection of $K_{[X / \mathcal{F}]}$ with $d \mu_{[X / \mathcal{F}]}$ is given by a residue around the singularities of $\mathcal{F}$, i.e. there is a one form (in fact many) $\omega$ smooth outside the singularities such that,

$$
K_{[X / \mathcal{F}]} \cdot d \mu_{[X / \mathcal{F}]}=\lim _{\epsilon \rightarrow 0} \int_{S(\epsilon)} \omega d \mu_{[X / \mathcal{F}]}
$$

where $S(\epsilon)$ is the boundary of a small ball around the singularities.

Plainly, this residue is simply a very slight generalisation of those appearing in I.3. It is, however, much more difficult to compute. Functorially understood, the winding number of a measure around the singularities is just the Le-long number, and so one might hope for,

Fact V.4.7 If the Le-long number of $d \mu_{[X / \mathcal{F}]}$ at every singularity of $\mathcal{F}$ is zero then the residue symbol in V.4.6 vanishes.

That this leads to a contradiction is exactly the argument of IV.5.7 given V.4.4. Better still, V.4.7 is easily seen to be trivial except for saddle nodes, so let's conclude this section with a proof of V.4.7 in this case which explicitly 
profits from the small ambient dimension, so unfortunately, only generalises to a particular category of isolated log-canonical singularities. Irrespectively, we may write our node as,

$$
\partial=y^{p+1} \frac{\partial}{\partial y}+(x+?) \frac{\partial}{\partial x}
$$

where ? indicates some stuff which is of order at least 2, and wholly irrelevant to our current considerations, and, of course $p \in \mathbb{N}$. By the very definition of Le-long number, and the comportment of saddles under blowing up, we can further suppose that $y=0$ is the local equation for an exceptional divisor $E$ in $X$ obtained by way of some initial blowing up, and $E \cdot d \mu_{[X / \mathcal{F}]}=0$. An inspection of the $\omega$ occurring in V.4.6 shows that it is more than sufficient to show,

$$
\lim _{\epsilon \rightarrow 0} \int_{\substack{|y|=\epsilon \\|x| \leq \epsilon}} \frac{d^{c}|y|}{|y|^{p+1}} d \mu_{[X / \mathcal{F}]}=0
$$

In fact, this is equivalent to showing that the residue of any bundle defined purely locally on the étale part of $[X / \mathcal{F}]$ around the node was zero. Now, our various hypothesis imply (rather trivially) that the residue of $d^{c} \log |y|$ around the singularity is zero, or equivalently,

$$
\lim _{\epsilon \rightarrow 0} \int_{\substack{|y|=\epsilon \\|x| \leq \epsilon}} \frac{d^{c}|y|}{|y|} d \mu_{[X / \mathcal{F}]}=\lim _{\epsilon \rightarrow 0} \frac{1}{2 \pi} \int_{\substack{|x|=\epsilon \\|y| \leq \epsilon}} \operatorname{Im}\left(y^{p} \frac{d x}{x}\right) d \mu_{[X / \mathcal{F}]}+?(\epsilon)
$$

where ? $(\epsilon)$ is some un-important error, of strictly smaller order in $\epsilon$ than the main term. The right hand side, however, is, by the vanishing of the Le-long number, a function of the form $\epsilon^{p} \Theta(\epsilon)$, where $\Theta(\epsilon)$ goes to zero with $\epsilon$, so, indeed, the desired residue is zero.

\section{V.5 Is there another way}

The final chapter of $[R]$, when reflecting on the classical Enriques-Kodaira theory of algebraic surfaces displays an odd dislike for Enriques lemma that a divisor of elliptic fibre type on a surface of numerical Kodaira dimension 1 must move, and asserts, without actually providing an answer, that there must be a better way to proceed. In the present foliated context, one might assert that the truth of all of V.3, and most of V.4 in all dimensions and it's very precise relation to abundance, or much more accurately establishing that non-general 
type foliations are truly special renders such a question irrelevant. To do so, however, would be to pass over the fact that the absolutely critical V.4.7 is achieved by a trick which is very particular to surfaces (or more generally certain types of isolated singularities, [M7] IV.3). As we've remarked in the introduction it was this very evident peculiarity in so much as it pertained to the proof of V.1.3, and the hope of eliminating it, that motivated a systematic study of foliated surfaces by Kodaira dimension to begin with. On the other hand, a wholly general approach to the higher dimensional variants of V.4.7 (which bears absolutely no relation to the above trickery) has now been provided, [M6] \& [M7], so, it's certainly true that the question is less pressing.

It's also true that there's absolutely no reason to believe that the basic philosophy encapsulated by V.4.4/5 isn't completely optimal, i.e. there may be details that one can improve on, but the intervention of the transverse dynamic as in V.4.7, or something similar, may very well be un-avoidable. To see this, following an initial enquiry of Bogomolov, let's consider what becomes of the arguments of V.4, and for that matter, V.1.3, in mixed characteristic. To begin with, we have,

Fact V.5.1 Let $S$ be finite over an open of $\operatorname{Spec} \mathbb{Z}$, and $\pi:(X, \mathcal{F}) \rightarrow S$ a family of foliated projective surfaces with canonical singularities at the generic point, then for every $\epsilon>0$, there is a proper sub-scheme $Z_{\epsilon}$ such that for any separable map $f: C \rightarrow X \otimes \overline{k(s)}$ from a smooth curve invariant by $\mathcal{F} \otimes \overline{k(s)}, s \in S$, not factoring through $Z_{\epsilon} \otimes \overline{k(s)}$,

$$
K_{\mathcal{F} \cdot f} C \leq(2 g(C)-2)+\epsilon H_{\cdot f} C
$$

where $H$ is relatively ample, i.e. V.4.2 continues to hold in mixed characteristic.

I've communicated a proof of this to Bogomolov, and in principle it ought to appear somewhere as part of a larger project for studying rational curves on surfaces. The curious reader who would like a proof now should look to how one proves V.4.2 in characteristic zero and dimension 3, and observe that the fundamental problem ain't that much different, i.e. there may be a countable set of $s$ such that the singularities of $\mathcal{F} \otimes k(s)$ are only log-canonical, then fill in a few details which are similar to II.1.3. Whence as a corollary,

Fact V.5.2 Hypothesis as in V.5.1, and suppose further that the generic fibre is a foliated surface of general type, then for all but finitely many $s$, the curves 
of genus $g$ on $X \otimes \overline{k(s)}$ invariant by $\mathcal{F} \otimes \overline{k(s)}$ are bounded in moduli and this bound is uniform in $s$, i.e. a part of V.1.3 but with $K_{\mathcal{F}}$ big remains true in mixed characteristic, e.g. for all but finitely many $s$ the invariant rational curves arise from specialisation of finitely many of the same at the generic point.

Now as it happens, the very fact that a huge set of fibres may have only logcanonical singularities, when viewed from the perspective of V.4.2 and its caveats, makes this pretty interesting of itself. It is however, even more instructive, to consider what happens to the remaining part of V.1.3. As ever, we may suppose that the generic fibre is a canonical, or even just minimal model, and generically we'll have our conditions $K_{\mathcal{F}}^{2}=0, K_{\mathcal{F}} \cdot K_{X}>0$ which lead to the hyperbolicity theorem V.1.3. Consequently, let's suppose that our corollary V.5.2 fails, so there would be a Zariski dense sequence of separable maps from smooth curves $f_{n}: C_{n} \rightarrow X \otimes \overline{k\left(s_{n}\right)}$ invariant by $\mathcal{F} \otimes \overline{k\left(s_{n}\right)}$ such that,

$$
\frac{\left(2 g\left(C_{n}\right)-2\right)}{H \cdot f_{n} C_{n}} \rightarrow 0 \quad \text { as } n \rightarrow \infty
$$

Next we'll want to mimic the construction of $d \mu_{[X / \mathcal{F}]}$ as a limit of cycles, but our possibilities for doing this are much less. For example, we can view the $C_{n}$ via duality as, not necessarily effective, classes in the $\mathrm{NS}_{1}(X \otimes K(S))$, i.e. NéronSeveri on the generic fibre, and normalise by the degree, and subsequence, so as to obtain a limit, $T$, in $\mathrm{NS}_{1}(X \otimes K(S)) \otimes \mathbb{R}$ which, by the way, is necessarily nef. Slightly better, we could perform the same procedure (use the crystaline Gysin map) in $H_{\mathrm{DR}}(X / S) \otimes \mathbb{C}$ after choosing an embedding of the generic point in an archimedean field, so as to conclude,

Fact V.5.3 Suppose as described above a mixed characteristic variant of V.1.3 fails then there is a canonical model of a foliation $(X, \mathcal{F})$ satisfying exactly the critical hypothesis of classification IV.5.8, i.e. $K_{\mathcal{F}}^{2}=0, K_{\mathcal{F}} . K_{X}>0$ such that the class of $K_{\mathcal{F}}$ in $H_{\mathrm{DR}}(X / S) \otimes \mathbb{C}$ is parallel to a closed $T$ arising from a normalised limit of invariant curves in positive characteristic, i.e. morally V.4.4 and its re-phrasing V.4.5 would appear to continue to hold.

The appearance however is more imagined than real, however, since in-fact,

Caveat V.5.4 Actually, unless a Zariski dense set of the curves $f_{n}: C_{n} \rightarrow$ $X \otimes \overline{k\left(s_{n}\right)}$ employed in the definition of $T$ occur at primes where $\mathcal{F} \otimes \overline{k\left(s_{n}\right)}$ is not 
just $p$-closed, but $p^{m}$ closed for all $m \in \mathbb{N}$, then apart from not actually being re-presented by a transverse invariant measure, it may not even be true that $T$ even looks like a foliated class in co-homology, i.e. without very strong hypothesis general crystaline apparatus does not allow us to conclude that $T$ is in the image of $\operatorname{Ext}^{1}\left(I_{Z}, K_{[X / \mathcal{F}]}\right)$, for $Z$ the singular sub-scheme of the foliation, cf. I.3., and even if it were, since it needn't be represented by an invariant meaure, $T$ could be very far from enjoying the special properties of its characteristic 0 analogue in V.4.4/5.

On, the other hand, the very hypothesis of V.5.3, irrespective of the existence of $T$, lead us by the classification theorem to deduce that our foliation must actually be one of the two naturally occurring foliations on the quotient of a bi-disc, so we can proceed to consider this mixed characteristic variant of V.1.3 rather explicitly. For simplicity of illustration let us suppose that $X$ is the quotient by a full $\mathrm{SL}_{2}(\mathcal{O})$ for $\mathcal{O}$ the ring of integers in a real quadratic field (the general case will be absolutely parallel), then in fact $S$ will be a localisation of $\operatorname{Spec} \mathcal{O}$, and the set of primes $p$ naturally divide according to,

(a) $p$ remains prime in $\mathcal{O}$, and neither of the natural foliations are $p$-closed, while for $p$ sufficiently large the specialised singularities, should they exist, will be canonical.

(b) $p$ splits as $\pi \bar{\pi}$, and both of the natural foliations aren't just $p$-closed but $p^{m}$-closed for all $m \in \mathbb{N}$, and $p$ sufficiently large, although the specialised singularities, should they exist, will only be log-canonical.

The behaviour of the singularities here, although we know that it can't be important by V.5.1 (and general nonsense always implies that its irrelevant to V.4.7 provided a log-canonical resolution exists) nevertheless serves to illustrate the optimality of our discussion, since in fact,

Fact V.5.5 Indeed, as implied by V.5.4, the invariant curves of genus $g$ on either of the natural foliations at primes $p$ of type (b) are, for $p$ sufficiently large, uniformly bounded in moduli as $p$ varies. For primes of type (a), however, the locus where the section of $K_{\mathcal{F}}^{p} \otimes K_{[X / \mathcal{F}]}^{\vee}$ defined by the $p$ th power map vanishes, cf. II.1, is supported on invariant rational curves, and the degree of these curves is unbounded in $p$. 
As a result, we are perfectly justified in making,

Summary V.5.6. The intervention of the transverse dynamic of the foliation in the proof of V.1.3 as found in V.4.7, or similar, would appear to be absolutely necessary. In addition, supposing V.4.1, or any other 'Schwarz type lemma' that one might care for such as the considerably stronger V.4.2, it is impossible to conclude to V.1.3 by way of any first order statement involving sections of jet bundles, or even, what is substantially stronger, sections of $K_{\mathcal{F}}$ and powers there of, i.e. one cannot prove V.1.3 by looking in the direction tangent to the foliation alone.

The impossibility indicated by V.5.6 is simply the consequence of V.5.5, and the fact that any first order statement would necessarily hold for all $p$ sufficiently large, and not just those of type (b). Indeed,

Alternative V.5.7 The so called Lang conjecture about rational curves on varieties of general type is false in mixed characteristic, i.e. there are surfaces whose generic fibre has general type, but on which the rational curves are Zariski dense.

All of which, largely settles, in the negative, any question as to whether there is a better way to prove V.1.3. It doesn't, however, settle whether the classification theorem could be proved in another way without V.1.3. Before even addressing this issue let us observe,

Further Caveats V.5.8 It isn't at all obvious that the classification theorem is even a first order statement, i.e. the Lefschetz principle may well not apply. The basic reason for this doubt is that there is no bound on the integer $n$ such that $K_{\mathcal{F}}^{\otimes n}$ is Cartier away from the egl's, cf. IV.4.4 (ii)(c), so that the classification theorem may well be a countable bunch of 1st order statements. Even worse, the classification theorem is a priori weaker than V.1.3, and although it would suffice for proving that the leaves were hyperbolic modulo $P$, it doesn't obviously imply the stronger statement of hyperbolicity modulo $B$ that is necessary for things such as the Green-Griffiths conjecture. In fact, even for Hilbert-Modular surfaces with singularities, the proof of the full V.1.3 still follows the schema of V.4, except that the singularities are so explicit that V.4.7 becomes trivial.

Consequently, if one is motivated primarily by hyperbolicity questions an alternative proof of classification not passing by V.1.3 is of limited value, albeit that 
the simplification that it brings to the transverse dynamics should by no means be underestimated. Irrespectively, plainly the intervention of the archimedean topology, and in particular of the Bochner formula, invites a parallel with Kodaira vanishing, and suggests that any other proof is likely to pass by way of positive characteristic. From this point of view II.2.2 is very much in the right direction, since under the usual hypothesis of IV.5.8, it would indeed imply $p$-closure for infinitely many $p$ if one knew that one singularity admitted an irrational eigenvalue. Unfortunately, this eigenvalue hypothesis, while a postiori true for cases of IV.5.8 not covered by IV.5.3, doesn't seem to admit an a priori proof. What's worse, is that it's far from clear that the $p$-closure statement (which incidentally would imply exactly the same simplifications to the proof of V.1.3 as the full classification theorem) is of itself sufficient to prove classification. Nevertheless, that the direction is correct follows from the classification of families of curves in positive characteristic as found in [S2], where the argument employed would actually generalise to foliations in characteristic zero if one had $p^{m}$-closure for all $m$, and certainly it works if one has infinitely many such $p$, albeit one might actually be enough.

Such, final considerations on alternative strategies for proving the classification theorem have for many years now been a favourite topic of conversation between Fedya and myself. Unfortunately, we've never quite been able to make it work, and the contents of this final section are, at least from what I have understood, a summary of these conversations, which may well be worth presenting to a wider audience in the (unlikely) event that we may have over looked some thing. I fear, though, that the very difficulty of V.1.3 demands that even if a characteristic $p$-alternative exists, it's likely to be anything but soft. 


\section{References}

[A] Artin M., On isolated rational singularities of surfaces, Amer. J. Math. 88 (1966), 129-136.

[B1] Bogomolov F., Holomorphic tensors and vector bundles on projective varieties, Math. USSR Izv. 13 (1978), 499-555.

[B2] Bogomolov F., Families of curves on surfaces of general type, Dokl. Akad. Nauk. SSSR 236 (1977), 1041-1044.

[BB] Baum P. and Bott R., Singularities of holomorphic foliations, J. Diff. Geom. 7 (1972), 279-342.

[Bl] Bloch A., Sur les systèmes de fonctions holomorphes à variétés linéaires lacunaires, Ann. École Norm. Sup. 43 (1926), 309-362.

[BM] Bogomolov F. and McQuillan M., Rational curves on foliated varieties, IHES Preprint, IHES/M/01/07 (2001).

[Br1] Brunella M., Feuilletages holomorphes sur les surfaces complexes compactes, Ann. Scient. École Norm. Sup. 30 (1997), 569-594.

[Br2] Brunella M., Courbes entières et feuilletages holomorphes, Enseign. Math. 45 (1999), 195-216.

[Br3] Brunella M., Minimal models of foliated algebraic surfaces, Bulletin S.M.F. 127 (1999), 289-306.

[Br4] Brunella M., Birational geometry of foliations, First Latin American Congress of Mathematicians, IMPA (2000).

[Br5] Brunella M., A subharmonic variation of the leafwise Poincaré metric, Invent. Math. 152 (2003), 119-148.

[Br6] Brunella, M. On the plurisubharmonicity of the leafwise Poincaré metric on projective manifolds, J. Math. Kyoto Univ. 45 (2005), 381-390.

[BPV] Barth W., Peters C., Van de Ven A., "Compact complex surfaces", SpringerVerlag, Berlin-New York 1984.

[C] Cartan, H. Sur les sytèmes de fonctions holomorphes à variétés linéaires lacunaires et leurs applications, Ann. École Normale 45 (1928), 255-346. 
[CI] Chirka, E. \& Ivashkovich, S., On the non-embeddability of Hartogs' figures into complex manifolds, . Bull. Soc. Math. France 134 (2006) 261-267.

[CKM] Clemens H., Kollár J., Mori S., Higher dimensional complex geometry, Astérisque 166 (1988).

[D] Demailly J.-P., $L^{2}$ vanishing theorems for positive line bundles and adjunction theory, Transcental methods in algebraic geometry.

[DV] Du Val J., Sur le lemme du Brody, Invent. Math., to appear.

[E1] Écalle J., Les fonctions resurgentes, Tome III, L'équation du pont et la classifcation analytique des objets locaux, Publ. Math. Orsay (1985).

[E] Ekedahl T., Foliations and inseparable morphisms, Proc. Sympos. Pure Math. 46 (1987).

[EST] Ekedahl T., Shepherd-Barron N.I. and Taylor R., A conjecture on the existence of compact leaves of algebraic foliations, Preprint.

[G1] Gromov, M. Spaces and questions, Geom. Funct. Anal. 13 (2003), 73-146.

[G2] Gromov, M. Pseudo-holomorphic curves in symplectic manifolds, Invent. Math. 82 (1985), 307-347.

[Gr] Green, M. The hyperbolicity of the complement of $(2 n+1)$ hyperplanes in general position in $\mathbb{P}^{n}$, and related results, Proc. Amer. Math. Soc. 66 (1977), 109-113.

[GG] Green M., Griffiths P., Two applications of algebraic geometry to entire holomorphic mappings, The Chern Symposium (1979).

[GM] Gomez-Mont X., Holomorphic foliations in ruled surfaces, Trans. A.M.S. 312 (1989), 179-201.

[Gro] Grothensieck, A. Cohomologie locale des faisceaux cohérents et Théorèmes de Lefschetz locaux et globaux, $S G A 2$.

[J] Jouanolou J.-P., Hypersurface solutions d'une équation de Pfaff analytique, Math. Ann. 232 (1978), 239-245.

[K1] Kollár J., "Rational curves on algebraic varieties", Springer-Verlag, Berlin 1996.

[K2] Kollár J., Flips and abundance for algebraic 3-folds, Astérisque 211 (1992). 
[Ka] Kawamata Y., The crepont blowing-up of 3-dimensional canonical singularities and its application to the degeneration of surfaces, Ann. Math. 127 (1988), 93-163.

[Ke] Keel S., Basepoint freeness for nef and big line bundles in positive characteristic, Ann. Math. 149 (1999) 253-286.

[KM] Keel, S., Mori, S., Quotients by groupoids, Ann. of Math. 145 (1997), 193-213.

[Kl] Kleiner B., Hyperbolicity using minimal surfaces, pre-print.

[KoN] Kobbayashi R., Naruki I., Holomorphic conformal structures and uniformisation of complex surfaces, Math. Ann. 279 (1988), 485-500.

[Kn] Knutson D., Algebraic spaces, L.N.M. 203 (1971).

[L] Lang S., Introduction to complex hyperbolic spaces, Springer-Verlag, New-York 1987.

[LC] Lang S., Cherry W., Topics in Nevanlinna Theory, L.N.M. 1433 (1990).

[M] Margulis G.A., Discrete subgroups of semisimple Lie groups, Springer-Verlag, New York 1991.

[M1] McQuillan M., Diophantine approximation and foliations, Publ. Math. I.H.E.S. 87 (1998), 121-174.

[M2] McQuillan, M. Integrating $\partial \bar{\partial}$, Proc. ICM, Vol. 1, 547-554, Higher Ed. Press, Peking, 2002.

[M3] McQuillan, M. Non-commutative Mori theory, IHES preprint IHES/M/ 00/15 (2000).

[M4] McQuillan, M. Non-commutative Mori theory, IHES preprint IHES/M/ 01/07 (2001).

[M5] McQuillan M., Semi-stable reduction of foliations, IHES pre-print IHES/ M/05/02 (2005).

[M6] McQuillan M., Categrical Integration, further appendix to revised version of [M5].

[M7] McQuillan M., Uniform Uniformisation, IHES pre-print IHES/M/05/ 03 (2005). $\dagger^{3}$

\footnotetext{
${ }^{3} \dagger$ Not only [M5], but also [M7] have been revised. In fact the on-line vesrion of the latter contains an error in IV.4-5, which the dual revision has corrected. The revised versions are in free circulation, and also available on request.
} 
[Ma] Martinet J., Normalisations de champs de vecteurs holomorphes (d'après Brujno), Sém. Bourbaki Exp. 564 (1980/81).

[MR] Martinet, J., \& Ramis J.-P., Problèmes de modules pour des équations différentielles non linéaires du premier ordre, Inst. Hautes Études Sci. Publ. Math. 55 (1982), 63-164.

[Me1] Mendes L.G., Kodaira dimension of singular holomorphic foliations, preprint Université de Dijon, april 1999.

[Me2] Mendes L.G., On algebraic and entire curves invariant by singular holomorphic foliations, preprint, 1999.

[Mi1] Miyaoka Y., Canonical degree of curves on a minimal surface, M.P.I. preprint, 1998.

[Mi2] Miyaoka Y., Deformations of morphisms along a foliation, Proc. Symp. Math. 46 (1987).

[Mi3] Miyaoka Y., Algebraic surfaces with positive index, Progress in Math. 39 (1983).

[Mi4] Miyaoka Y., The maximal number of quotient singularities on surfaces with given numerical invariants, Math. Ann. 268 (1984), 159-171.

[Mo] Mok, N. Holomorphic foliations with compatible degenerate Kahler metrics: applications to the Shafarevich conjecture and to fibration theorems, Geometric complex analysis (Hayama 1995) 475-484, World Sci. Publishing, River Edge 1996.

[Mu] Mumford D., The topology of normal singularities on algebraic surfaces, Publ. Math. I.H.E.S. 9 (1961), 5-22.

[P] Pereira J. V., On the height of foliated surfaces with vanishing Kodaira dimension, Publicacions Matemàtiques 49 (2005) 363-373.

[R] Reid M., Chapters on algebraic surfaces in Complex Algebraic Geometry, LAS/Park City Math. Ser. 3, AMS, Providence 1997.

[RS] Rudakov, A., \& Shafarevich, I.R., Inseparable morphisms of algebraic surfaces, Izv. Akad. Nauk. SSR 40 (1976) 1269-1307.

[S] Seidenberg A., Reduction of singularities of the differential equation $A d y=B d x$, Amer. J. Math. 89 (1967), 248-269.

[S-B] Shepherd-Baron N., Miyaoka's Theorems, Astérisque 211 (1992), 103-114. 
[Si1] Siu Y.-T., Analycity of sets associated to Lelong numbers and extensions of closed positive currents, Invent. Math. 27 (1974), 53-156.

[Si2] Siu Y.-T., A vanishing theorem for semi-positive line bundles over non-Kähler manifolds, J. Differ. Geom. 19 (1984) 431-452.

[Sik] Sikorav, J.-C. Some properties of holomorphic curves in almost complex manifolds, Progress in Math. 117 (1994), 165-189.

[S1] Szpiro, L., Sur le thorme de rigidit de Parshin et Arakelov, Astérisque, 64 (1974), 169-202.

[S2] Szpiro L., Séminaire sur les Pinceaux des Courbes de Genre au Moins Deux, Astérisque $\mathbf{8 6 .}$.

[TY] Tian G., Yau S.-T., Existence of Kahler-Einstein metrics on complete Kahler manifolds and their applications to algebraic geometry, Adv. Ser. Math. Phys. 1 (1987).

[V] Vojta P., On algebraic points on curves, Comp. Math. 78 (1991), 29-36.

[Y] Yamaguchi, H. Calcul des variations analytiques, Jap. J. Math. 7 (1981), 319-377.

[ZS] Zariski O. and Samuel P., Commutative Algebra Vol. II, D. Van Nostrand and Co., Princeton, Toronton, London, New York 1960.

Michael McQuillan

E-mail: mquillan@ihes.fr 